PLASMONIC AU NANOSTRUCTURES FOR SURFACE-ENHANCED RAMAN SPECTROSCOPY

A Dissertation Presented to the Faculty of the Graduate School University of Missouri

In Partial Fulfillment

of the Requirements for the Degree

Doctor of Philosophy

By
XIN SUN
Dr. Hao Li, Dissertation Supervisor
December 2013


(C) Copyright by Xin Sun 2013

All Rights Reserved 


\section{Dedications}

This dissertation is dedicated to my daughter, Mia Sun, my wife, Mrs. Ji Sun, my parents, Mr. Xianglian Sun and Mrs Lijuan Zhang, and my parents in law, Mr Xiulin Sun and Mrs Zhongyan Guo, for their encouragement, support, and love. 


\section{ACKNOWLEDGEMENTS}

Pursuing my $\mathrm{PhD}$ degree in mechanical and aerospace engineering has been a long and challenging journey. I was, however, fortunate to have the assistance, encouragement, and support of many people throughout my doctoral study. Although it would be impossible to name every single person and his/her contribution, I would like to express my sincere appreciation and thanks to each of them.

First, I would like to sincerely thank my advisor, Dr. Hao Li, for his tremendous time and effort he has spent in leading, encouraging, and supporting me during last five years. I am so blessed to have a respectable and admirable supervisor who has inspired me by his passion for overcoming challenges. Through his knowledge of science and perseverance in research he has guided me through my career and has given me confidence to succeed. Thank you again for your unlimited understanding, encouragement and support.

I would like to express my gratitude to all of the committee members, Dr. Qingsong Yu, Dr. Mengshi Lin, Dr. Stephen Lombardo, and Dr. Zhiqiang $\mathrm{Hu}$, for participating in my comprehensive examination and dissertation defense. Special thanks must go to Dr. Mengshi Lin for providing valuable guiding and advising in applying SERS technology to food science applications. I am also grateful to Dr. Qingsong $\mathrm{Yu}$ for his generous 
suggestions and instrumental support in the research of plasma nanocoating. I also would like to take this opportunity to acknowledge all my current and former colleagues Dr. Meng Chen, Dr. Andrew Ritts, Dr. Young Jo Kim, Dr. Liang Chen, Dr. Jian Shi, Dr. Wen Wang, Dr. Lili He, Dr. Bin Liu, Dr. Adam Blumhagen, Dr. Da Yan, Dr. Xiaoqing Dong, Mr. Richard Lebens, Mr. John Jones, Mr. Qing Hong, Mr. Nan Wang, Mr. Huibin Chang, Ms. Dan Liu, Ms. Kathryn Giddens, Mr. Xuesong Song, Mr. Alan McGinnis, Mr. Jonathan Schottler, and Mr. Christopher Nassau for their collaboration, discussions, assistance and making such a joyful work and research environment during these years. I am also very grateful to the staff, Dr. Tommy White, Mr. Louis Ross Jr, Mr Randy Tindall, Ms. Cheryl Jensen, and Ms Melainia L. McClain from Electron Microscopy Core for their assistance on scanning/transmission electron microscopy characterization. 


\section{TABLE OF CONTENTS}

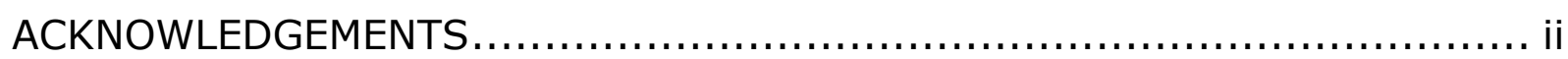

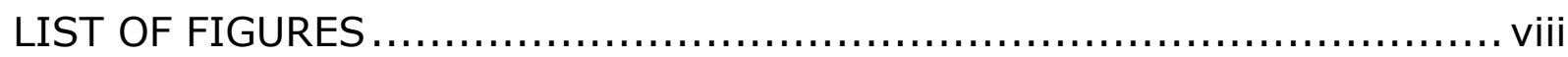

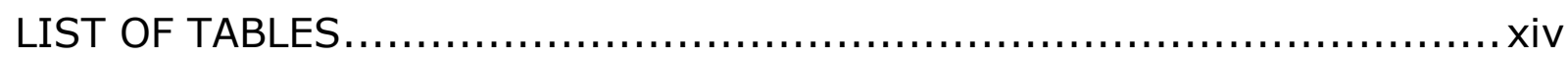

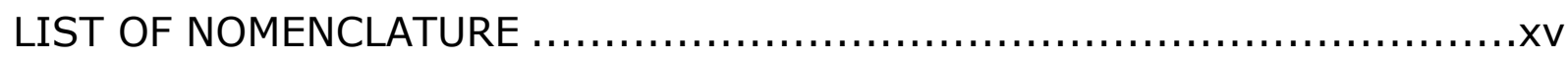

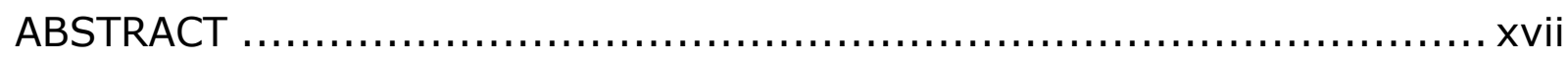

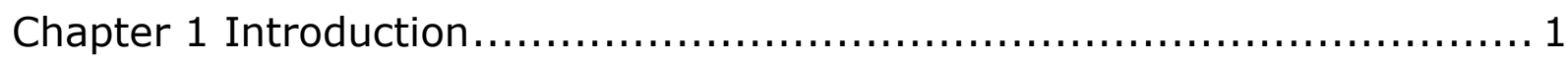

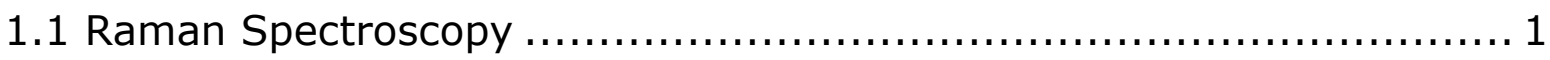

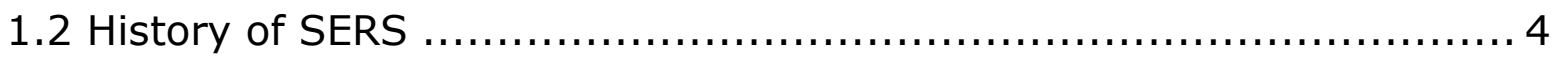

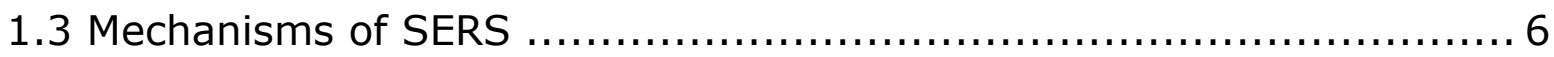

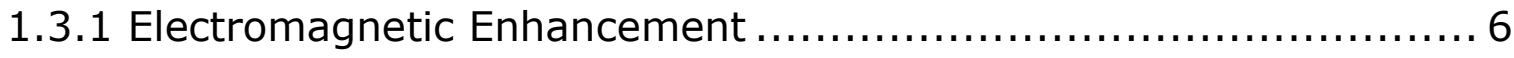

1.3.2 Chemical Enhancement.............................................. 8

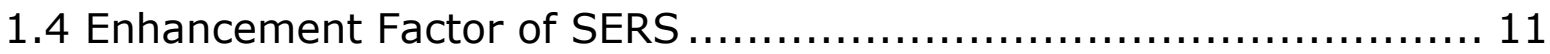

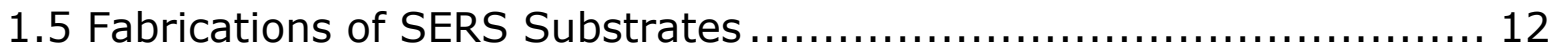

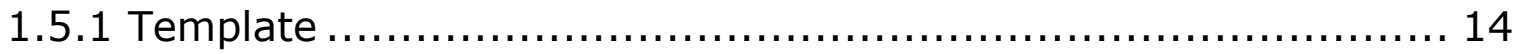

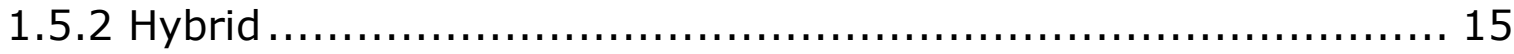

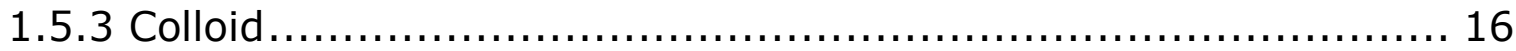

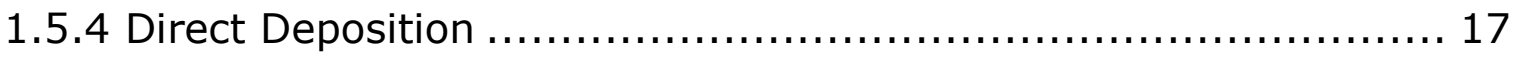

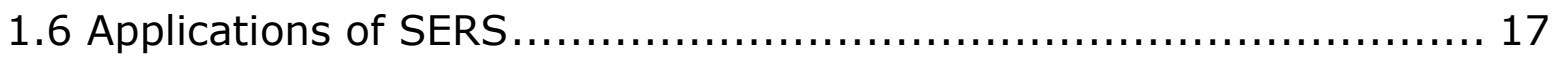

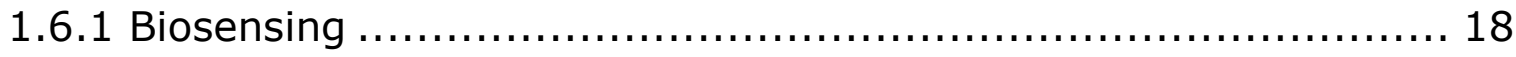

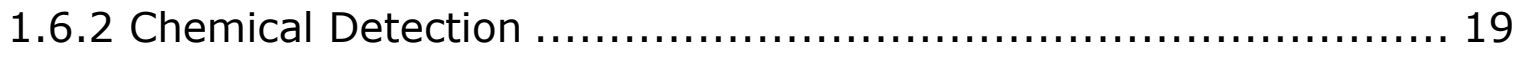

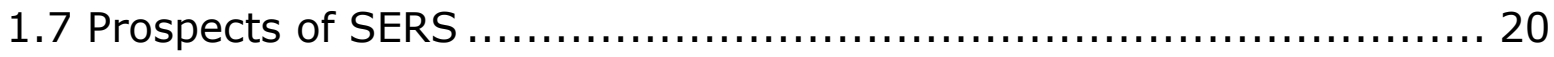

1.8 Objectives of Research ............................................... 21

1.9 Outline of Dissertation .............................................. 22 
1.10 Reference.

Chapter 2 Horizontally Aligned One-Dimensional ZnO Nanonecklaces by CVD for SERS 38

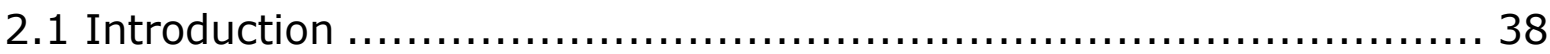

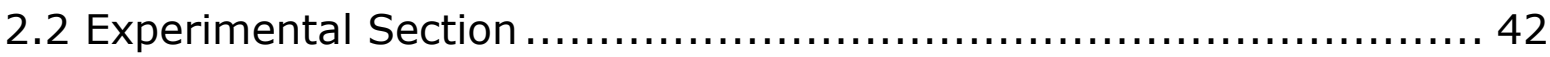

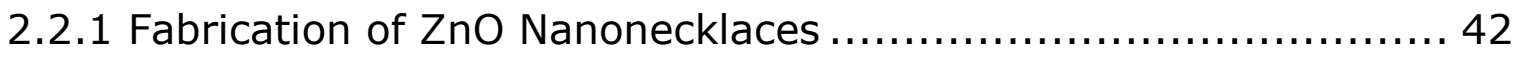

2.2.2 Characterization of ZnO Nanonecklaces........................ 44

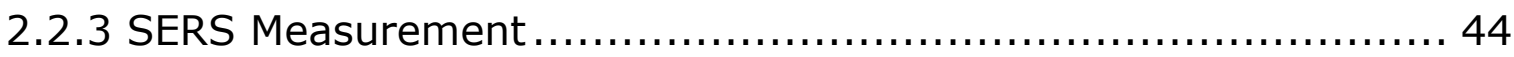

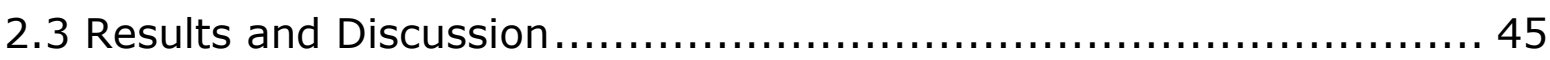

2.3.1 Morphology of ZnO Nanostructures........................... 45

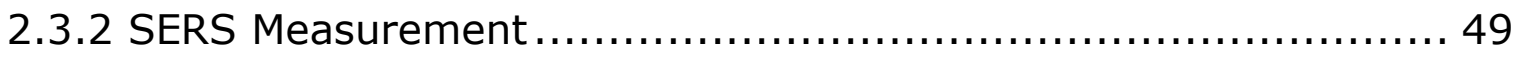

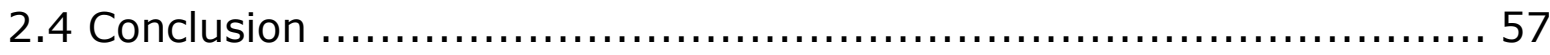

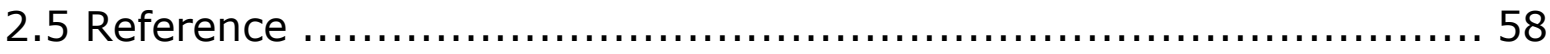

Chapter 3 Au Nanoisland Arrays by Repeated Deposition and Post-Deposition

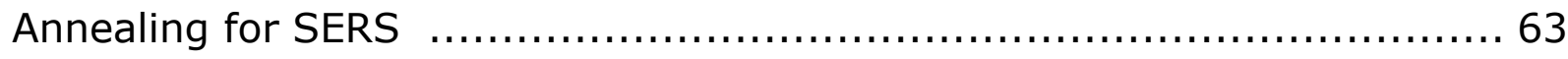

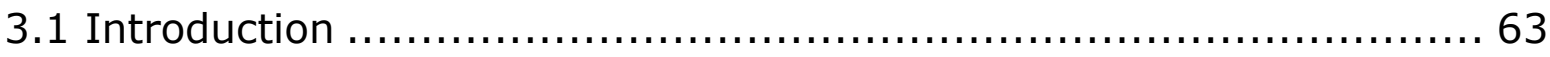

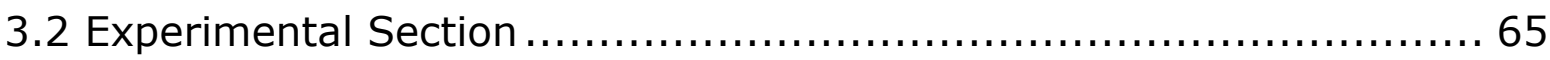

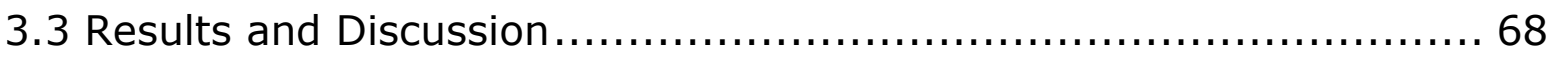

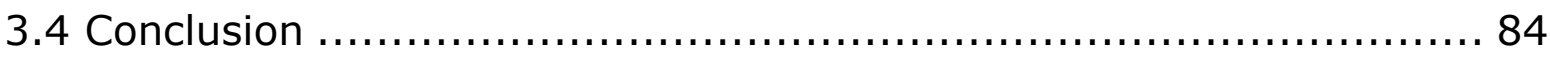

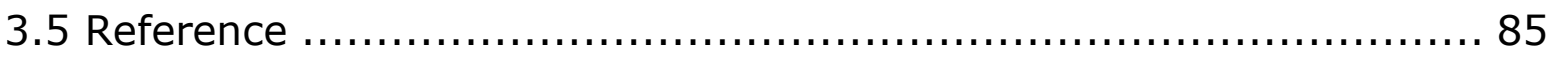

Chapter 4 Deep Etched Porous Si Decorated with Au Nanoparticles for SERS 91

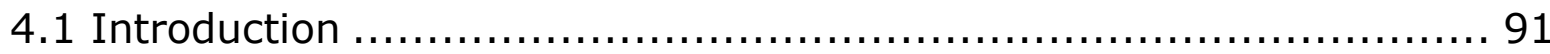

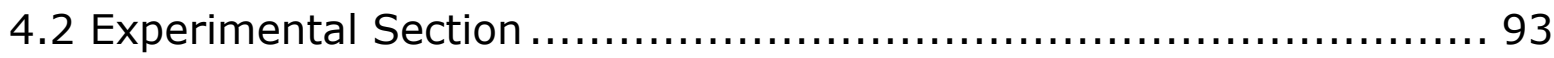

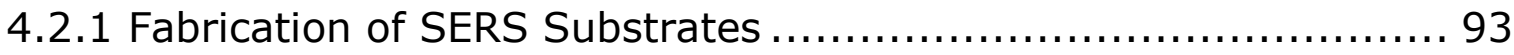




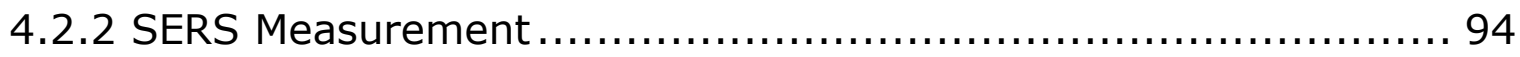

4.2.3 Effects of Predefined Au Nanoisland Arrays by Annealing ......... 95

4.3 Results and Discussion............................................. 95

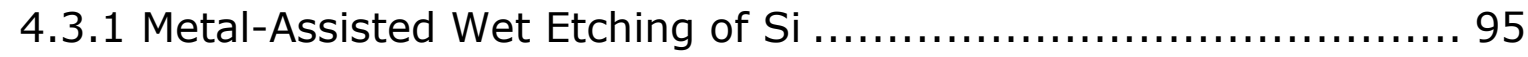

4.3.2 Morphology of SERS Substrates .................................. 97

4.3.3 SERS Dependence on Au Coating Thickness and Etching Time...100

4.3.4 Evaluations of Enhancement Factor and Uniformity ..............107

4.3.5 Effects of Predefined Au Nanoisland Arrays by Annealing .........110

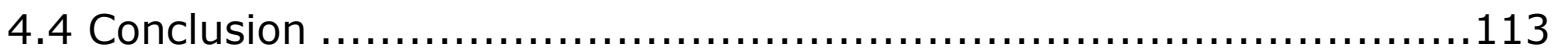

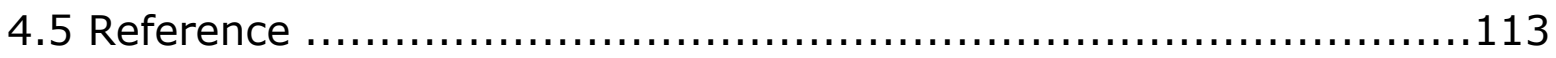

Chapter 5 Influence of Trimethylsilane Plasma Coating on SERS Substrates and the Commercialization Potential of Plasma Coated SERS Substrates .118

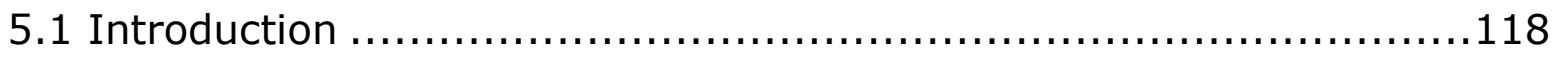

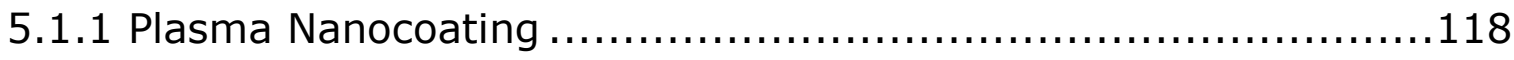

5.1.2 Commercialization of SERS Substrates ............................120

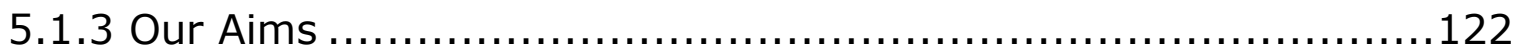

5.2 Experimental Section ............................................... 124

5.2.1 Fabrication of Plasma Coated SERS Substrates....................124

5.2.2 Characterization of Plasma Coated SERS Substrates $\ldots \ldots \ldots \ldots \ldots 125$

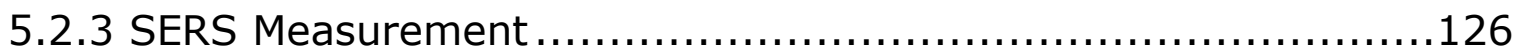

5.2.4 Sample Preparation for Carbaryl Detection from Polluted Fruits 127

5.3 Results and Discussion ...............................................

5.3.1 Influence of Plasma Nanocoating on SERS Substrates ............128

5.3.2 Commercialization Potential of Plasma Coated SERS Substrates 134 


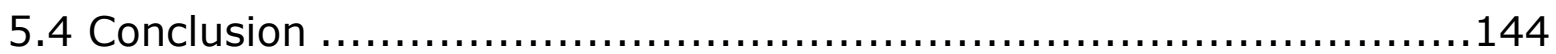

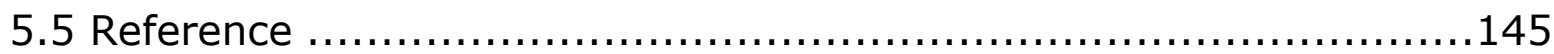

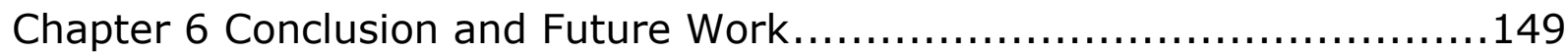

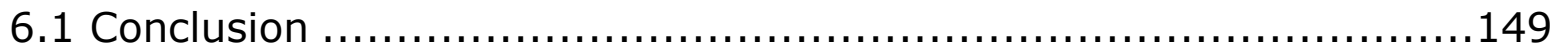

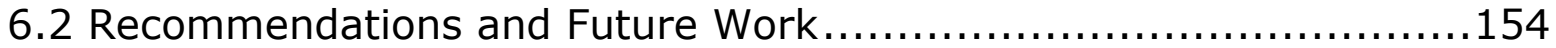

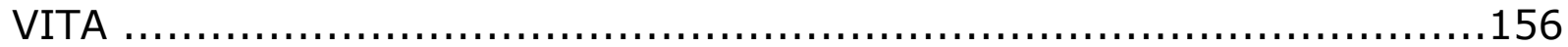




\section{LIST OF FI GURES}

Figure

Page

1.1 Illustration of possible energy transfers during a scattering process between a molecule and an incident photon. 3

1.2 Energy level diagram for a molecule adsorbed on metal surface. Possible charge transfer excitations are indicated with arrows 10

2.1 The schematic CVD reactor setup for $\mathrm{ZnO}$ NNs growth 43

2.2 FESEM images of ZnO NNs fabricated at position A ((a) and (b)), C ((c) and (d)) and E ((e) and (f)); ( $g$ ) the boundary between Au nanoparticles uncoated and coated regions of sapphire substrate, leading to ZnO NNs free and present regions, respectively; $(\mathrm{h}) \mathrm{ZnO}$ nanoblades 46

2.3 (a) The length and width of ZnO NNs vs the distance between precursors and substrates; (b) the length and width of $\mathrm{ZnO}$ NNs vs the growth time.. 48

2.4 AFM image (a) and section analysis profile (b) of the ZnO NNs..... 49

2.5 Average Raman spectra $(n=3)$ of $10^{-4} \mathrm{~mol} / \mathrm{L}$ melamine on a ZnO NN substrate with $45 \mathrm{~nm}$ (a) and $60 \mathrm{~nm}$ (b) Au coating. Raman measurements were taken under the same conditions 50

2.6 Average Raman spectra $(n=3)$ of $10^{-4} \mathrm{~mol} / \mathrm{L}$ melamine on a Au coated ZnO NN substrate and $10^{-2} \mathrm{~mol} / \mathrm{L}$ melamine (inset) on a Au film substrate. Raman measurements were taken under the same conditions 51

2.7 Average SERS spectra of a serial concentration of melamine $\left(a: 10^{-2}, b\right.$ : $10^{-3}$, c: $10^{-4}, \mathrm{~d}: 10^{-5}$, and e: $10^{-6} \mathrm{~mol} / \mathrm{L}$ ) deposited onto Au coated ZnO NNs 53 
2.8 Relationship between log value of melamine concentration ( $\mathrm{mol} / \mathrm{L}$ ) and Raman intensity of a melamine peak at $683 \mathrm{~cm}^{-1}$ (average of three spectra) 54

2.9 Average Raman spectra ( $\mathrm{n}=3$ ) of $10^{-4} \mathrm{~mol} / \mathrm{L}$ malachite green (a) and $10^{-4} \mathrm{~mol} / \mathrm{L}$ crystal violet (b) on an Au coated ZnO NN substrate 54

2.10 SEM images of $\mathrm{ZnO}$ nanoblades and nanonecklaces grown on sapphire substrate that was $20 \mathrm{~mm}$ away from the precursors 56

2.11 Raman intensity of melamine $\left(10^{-4} \mathrm{~mol} / \mathrm{L}\right)$ peak at $683 \mathrm{~cm}^{-1}$ acquired from five different $\mathrm{ZnO} \mathrm{NN}$ substrates (average of three spectra). All substrates were coated with $\sim 45 \mathrm{~nm}$ Au coating .......................... 57

3.1 Schematic fabrication process of SERS substrates 65

3.2 SEM images of Au nanoisland arrays at different stages during growth. Morphology of nanoisland arrays after $1^{\text {st }}(a), 2^{\text {nd }}(c)$, and $3^{\text {rd }}(e)$ sputtering. Morphology of nanoisland arrays after $1^{\text {st }}(b), 2^{\text {nd }}(d)$, and $3^{\text {rd }}(f)$ annealing. Alternatively, (b), (d), and (f) represent single, double, and triple processed Au nanoisland arrays, respectively. All scale bars equal to $200 \mathrm{~nm}$.........6 69

3.3 Histogram of nanoislands size distribution after single, double, and triple deposition and annealing processes 70

3.4 AFM images of single (left), double (middle), and triple (right) processed Au nanoisland arrays 71

3.5 SEM images of $10 \mathrm{~nm}$ and $15 \mathrm{~nm}$ Au thin films before and after annealing on Si substrate. (a) $10 \mathrm{~nm}$ before annealing; (b) $15 \mathrm{~nm}$ before annealing; (c) $10 \mathrm{~nm}$ after annealing; (d) $15 \mathrm{~nm}$ after annealing. All scale bars equal to $200 \mathrm{~nm}$ 72 
3.6 SERS spectra of $2 \mathrm{ppm}$ MG on single, double, and triple processed Au nanoisland arrays 75

3.7 (a) SERS spectra of $200 \mathrm{ppb}$ MG on triple processed Au nanoislands arrays with $0 \mathrm{~nm}, 10 \mathrm{~nm}, 20 \mathrm{~nm}, 30 \mathrm{~nm}, 40 \mathrm{~nm}, 50 \mathrm{~nm}$, and $60 \mathrm{~nm} \mathrm{Au}$ depositions. (b) Relationship between normalized intensity at $v=1173 \mathrm{~cm}^{-1}$ and Au deposition thickness

3.8 SEM images of triple processed Au nanoisland arrays with $20 \mathrm{~nm}$ (a), 40 $\mathrm{nm}$ (b), and $60 \mathrm{~nm}$ (c) Au depositions, respectively. Marks in (b) and (c) show the formation of bigger islands. Simulation of scattered electric field induced by a vertical incident plane wave $(\lambda=785 \mathrm{~nm})$ on a smooth sphere (d) and a secondary nanoparticle-coated sphere (e)........................ 79

3.9 (a) SERS spectra of a series of low level MGs and a blank reference collected on a triple processed Au nanoisland arrays with $40 \mathrm{~nm}$ gold deposition. (b) Relationship between Raman intensity at $v=1615 \mathrm{~cm}^{-1}$ and concentration of analyte solution 81

3.10 SERS spectrum of $10^{-5} \mathrm{M}$ BDT acquired on triple processed nanoisland arrays with $40 \mathrm{~nm}$ Au coating (top). Raman spectrum of $10^{-1} \mathrm{M}$ BDT acquired on $50 \mathrm{~nm}$ Au film on un-etched $\mathrm{Si}$ (bottom)

3.11 The distribution of Raman intensity $\left(v=1030 \mathrm{~cm}^{-1}\right.$ of $\left.10^{-4} \mathrm{M} \mathrm{BDT}\right)$ over a $5 \mathrm{~cm}$ by $5 \mathrm{~cm}$ SERS substrate (same recipe as the one in Fig. 3.9) divided with 100 equal-sized grids 84

4.1 Top view SEM images of Au nanoislands arrays (a) and porous $\mathrm{Si}$ fabricated from (a) with $0.5 \mathrm{~min}$ (b), $5 \mathrm{~min}$ (c) and $10 \mathrm{~min}$ (d) etching periods. Cross sectional SEM image of 5 min etched porous Si (inset of c). 97 
4.2 Top row: top view SEM images of $30 \mathrm{~nm}$ (a), $90 \mathrm{~nm}$ (b), $150 \mathrm{~nm}$ (d), and $300 \mathrm{~nm}$ (d) Au coatings on $0.5 \mathrm{~min}$ etched porous $\mathrm{Si}$, respectively. Middle row: top view SEM images of $30 \mathrm{~nm}$ (e), $90 \mathrm{~nm}$ (f), $150 \mathrm{~nm}$ (g), and $300 \mathrm{~nm}(\mathrm{~h})$ Au coatings on $10 \mathrm{~min}$ etched porous Si, respectively. Bottom row: top view SEM images of $30 \mathrm{~nm}$ (i), $90 \mathrm{~nm}$ (j), $150 \mathrm{~nm}(\mathrm{k})$, and $300 \mathrm{~nm}$ (I) Au coatings on 30 min etched porous Si, respectively 99

4.3 Au coating thickness dependence of Raman intensity $\left(v=1030 \mathrm{~cm}^{-1}\right.$ of $10^{-4} \mathrm{M} \mathrm{BDT}$ ) 102

4.4 Relationship between root mean square (RMS) surface roughness and $\mathrm{Au}$ coating thickness for three representative etching periods $(0.5 \mathrm{~min}, 10 \mathrm{~min}$, and $30 \mathrm{~min}$ ) 103

4.5 Etching time dependence of Raman intensity $\left(v=1030 \mathrm{~cm}^{-1}\right.$ of $\left.10^{-4} \mathrm{M} \mathrm{BDT}\right)$ 105

4.6 (a) Contour map of Raman intensity at $v=1030 \mathrm{~cm}^{-1}$ of $10^{-4} \mathrm{M}$ BDT solution with respect to two parameters: Au coating thickness and etching time. (b) SEM image of SERS substrate with optimized parameters: $40 \mathrm{~min}$ etched porous Si and $200 \mathrm{~nm}$ Au coating. Scale bar represents $1 \mu \mathrm{m}$ 107

4.7 SERS spectrum of $10^{-5} \mathrm{M}$ BDT acquired on 20 min etched porous Si with $150 \mathrm{~nm}$ Au coating (red). Raman spectrum of $10^{-1} \mathrm{M}$ BDT acquired on $50 \mathrm{~nm}$ Au film on un-etched Si (black) 108

4.8 Raw SERS spectra of $10^{-5} \mathrm{M}$ BDT collected at 20 random spots on the SERS substrate of Fig. 4.7. 110

4.9 (a) Relationship between Raman intensity ( $v=1030 \mathrm{~cm}^{-1}$ of $10^{-4} \mathrm{M}$ BDT) and $\mathrm{Au}$ coating thickness for SERS substrates based on 10 min etched porous Si. Red and blue curves represent SERS substrates etched from 
annealed and un-annealed substrates, respectively. (b) RSD of Raman intensity among three batches of experiments shown in (a). Red and blue bars represent SERS substrates etched from annealed and un-annealed substrates, respectively .112

5.1 The design of combining plasma nanocoating with regular SERS substrates fabrication

5.2 Thickness of plasma nanocoating on bare Si wafers with different plasma treatment time

5.3 Digital photograph of water droplets on uncoated (left) and plasma coated (right, $50 \mathrm{~nm}$ in thickness) SERS substrates.

5.4 SEM images of SERS substrates before and after plasma nanocoating (50 $\mathrm{nm}$ in thickness)

5.5 Relative peak intensity comparison at Raman shift of $1379 \mathrm{~cm}^{-1}$ using uncoated and plasma coated SERS substrates. The analyte was 100 ppm carbaryl in a solvent mixture of acetonitrile and deionized water (equal volume ratio). For each substrate, two measurements, one at the center and the other one at the edge of analyte residue area, were conducted

5.6 Schematic illustration of the influence of plasma nanocoating on droplet shape and residue shape and size. Please note the laser beam size, $\sim 5 \mu \mathrm{m}$ in our case, is much smaller than the residue size

5.7 SERS spectra of $500 \mathrm{ppb}$ (red) and $100 \mathrm{ppb}$ (blue) $\mathrm{BCL}$ on plasma coated SERS substrate; black spectrum represents $100 \mathrm{ppb} B C L$ on a commercial SERS product 136 
5.8 SERS spectrum of $10^{-5} \mathrm{M}$ BDT acquired on plasma coated SERS substrates (top). Raman spectrum of $10^{-1} \mathrm{M}$ BDT acquired on $50 \mathrm{~nm}$ Au film on un-etched Si (bottom)

5.9 Top: Recorded peak intensities of $683 \mathrm{~cm}^{-1}$ (2.5 and $1 \mathrm{ppm}$ melamine) for ten plasma coated SERS substrates. Bottom: Recorded peak intensities of $683 \mathrm{~cm}^{-1}$ ( $1 \mathrm{ppm}$ melamine) on ten substrates that were operated by three operators.

5.10 (a) SERS spectra of $0.5 \mathrm{ppm}$ malachite green on plasma coated SERS substrates after exposed in ambient for up to 12 months. (b) Raman intensity of a selected peak $\left(1173 \mathrm{~cm}^{-1}\right)$ after exposed in ambient for different periods.

5.11 Average SERS spectra $(n=8)$ of carbaryl solutions with different concentrations (in ppm). Spectra were presented with smoothing at $4 \mathrm{~cm}^{-1}$ and baseline adjustment by subtracting a 2 nd order polynomial function .143 


\section{LIST OF TABLES}

Table

Page

2.1 Crystallographic relationships between $\mathrm{ZnO}$ and sapphire substrates .. 40

5.1 Surface energy of liquid used to calculate surface energies of uncoated and TMS plasma coated SERS substrates. The units are $\mathrm{mJ} / \mathrm{m}^{2}$. Corresponding values are taken from the reference [28]

5.2 The contact angles ( $\cos \theta)$ and their surface energies of uncoated and TMS plasma coated (thickness $=10,20,50,100 \mathrm{~nm}$ ) SERS substrates. Surface energy of each liquid is $\mathrm{YL}^{+}=25.5,0,1.92, \mathrm{YL}^{-}=25.5,0,47.0$, and $\mathrm{YL}^{\mathrm{LW}}=21.8,50.8,29.0$ for water, diiodomethane and ethylene glycol respectively.[28] $\mathrm{Ys}^{+}$and $\mathrm{Ys}^{-}$are the surface energies contributed from electron acceptors and donors. $\mathrm{Y}^{\mathrm{LW}}$ and $\mathrm{Y}^{\mathrm{AB}}$ are surface energies from lifshitzvan der Waals interaction and Lewis acid-base interaction

5.3 Sensitivity of plasma coated and uncoated SERS substrates to various chemicals on two Raman spectrometers 138

6.1. Performance of four plasmonic Au nanostructures in reference to SERS evaluation criteria 


\title{
LI ST OF NOMENCLATURE
}

\author{
$A B=$ acid-base \\ $A E F=$ analytical enhancement factor \\ AFM=atomic force microscopy \\ $\mathrm{BCL}=$ brilliant cresyl blue \\ $\mathrm{BDT}=1,2$-benzenedithiol \\ $\mathrm{CHEM}=$ chemical enhancement \\ $\mathrm{CV}=$ crystal violet \\ $C V D=$ chemical vapor deposition \\ $\mathrm{DI}=$ de-ionized \\ DNT = dinitrotuluene \\ $E B L=$ electron beam lithography \\ $\mathrm{EF}=$ enhancement factor \\ $E M=$ electromagnetic enhancement \\ FDA $=$ Food and Drug Administration \\ FESEM = field-emission scanning electron microscopy \\ FTIR=Fourier transform infrared spectroscopy \\ $\mathrm{HOMO}=$ highest occupied molecular orbital \\ LSPR $=$ localized surface plasmon resonance \\ LW $=$ Lifshitz-van der Walls \\ $\mathrm{LUMO}=$ lowest unoccupied molecular orbital \\ $M G=$ malachite green
}




$$
\begin{aligned}
& \mathrm{NB}=\text { nanoblade } \\
& \mathrm{NN}=\text { nanonecklace } \\
& \text { NSL= nanosphere lithography } \\
& \mathrm{OAD}=\text { oblique angle deposition } \\
& \text { ppm }=\text { parts per million } \\
& \mathrm{ppb}=\text { parts per billion } \\
& \mathrm{PS}=\text { polystyrene } \\
& \text { PVD }=\text { physical vapor deposition } \\
& \mathrm{PVP}=\text { polyvinylpyrrolidone } \\
& \mathrm{RF}=\text { radio frequency } \\
& \text { RMS=root mean square } \\
& \mathrm{RSD}=\text { relative standard deviation } \\
& \text { SAM }=\text { self assembled monolayers } \\
& \text { sccm =standard cubic centimeters per minute } \\
& \text { SEM=scanning electron microscopy } \\
& \text { SERS =surface-enhanced Raman spectroscopy } \\
& \text { TEM = transmission electron microscopy } \\
& \text { TMS }=\text { trimethylsilane } \\
& \mathrm{TNT}=\text { trinitrotoluene } \\
& \mathrm{UV}=\text { ultraviolet } \\
& \text { VLS=vapor-liquid-solid } \\
& \mathrm{XPS}=\mathrm{x} \text {-ray photoelectron spectroscopy }
\end{aligned}
$$




\title{
Plasmonic Au Nanostructures for Surface-Enhanced
}

\section{Raman Spectroscopy}

\author{
Xin Sun \\ Dr. Hao Li, Dissertation Supervisor
}

\begin{abstract}
Raman spectroscopy is a spectroscopic technique that provides rich structural information for identifying chemical species but finds limited applications owing to its low sensitivity. Surface-enhanced Raman spectroscopy (SERS) is capable of solving the issue of sensitivity by enormously amplifying the Raman signal through localized surface plasmon resonance (LSPR) that is induced by so-called plasmonic nanostructures. Since the inception of SERS in 1970s, significant efforts have been put in developing SERS-active substrates with high quality in terms of sensitivity, reliability, reproducibility, scalability, throughput, and cost. At present, however, SERS substrates with sufficiently high quality for both research activities and real-world applications have not stood out yet.
\end{abstract}

In this dissertation, four types of plasmonic Au nanostructures will be reviewed with respects to fabrication, characterization, optimization, and evaluation for SERS applications. Firstly, faceted $\mathrm{ZnO} / \mathrm{Au}$ nanonecklace 
arrays epitaxially grown on r-plane sapphire substrates by chemical vapor deposition and sputtering will be introduced. Secondly, Au nanoisland arrays prepared by repeated sputtering and post-deposition annealing will be presented. Thirdly, nanoporous $\mathrm{Si} / \mathrm{Au}$ composites resulting from metalassisted wet etching and sputtering will be reported. Lastly, we will present a novel plasma nanocoating technique that overcoats $\mathrm{SiO}_{2} / \mathrm{Au}$ SERS-active nanostructures with an ultra thin polymer layer, followed by the demonstration of benefits brought by such plasma nanocoating. The properties and growth mechanisms of abovementioned plasmonic Au nanostructures were investigated with scanning electron microscopy (SEM), atomic force microscopy (AFM), transmission electron microscopy (TEM), ellipsometry and contact angle analyzer. By correlating the enhancement of Raman signal with the experimental parameters, recipes for optimized plasmonic nanostructures were established. Furthermore, the applicability of these plasmonic Au nanostructures for SERS purposes was demonstrated by successfully detecting various chemical species at trace level. At the end of the dissertation, a brief summary on these four plasmonic Au nanostructures will be reviewed against the standards of high quality SERS substrates and corresponding recommendations will be proposed to further improve the SERS performance. 


\section{Chapter 1-Introduction}

Surface-enhanced Raman spectroscopy (SERS), an extension of standard Raman spectroscopy, has exhibited great potential in detecting trace amount of molecules with the aid of noble metal nanostructures. Translating this promising phenomenon into practical applications has been the focal point of my doctoral study. This introductory chapter starts with a brief introduction of Raman spectroscopy, followed by a comprehensive background information and literature review on SERS regarding to its history, mechanisms, characterizations, fabrications, applications, and prospects. At the end of the chapter, the objectives and outline of this study will be presented.

\subsection{Raman spectroscopy}

Raman spectroscopy [1-3] is a spectroscopic technique that is based on energy transfers while a molecule scatters an incident photon. 
As shown in Fig. 1.1, when a photon hits a molecule, the energy state of the molecule will be excited to a higher level. In most cases, the exited molecule will recover to its original energy level and no net energy transfer occurs during the process. And this elastic scattering is commonly known as Rayleigh scattering. However, in some rare cases, namely, one out of $10^{7}$, the excited molecule may regress to a different energy level, either higher or lower than its original one, which means the scattered photon may carry less (Stokes shifted) or more (anti-Stokes shifted) energy than it does prior to the scattering. This inelastic scattering is usually referred to as Raman scattering and it is highly useful as the energy transfer of Raman scattering process corresponds to a specific transition of vibrational, rotational or other low-frequency mode in a molecule. As a result, Raman scattering can be used as the corner stone of an analytical chemistry tool.

Raman spectroscopy, built on Raman scattering, shows exceptional features in providing rich structural information of the targeted molecules and realizing the identifications of molecules. In addition, compared to traditional analytical technique, for example, Fourier transform infrared spectroscopy (FTIR), Raman spectroscopy has an edge in that water molecule does not interfere with Raman scattering as it usually does with FTIR. All these features entitle Raman spectroscopy a suitable technique for the microscopic 


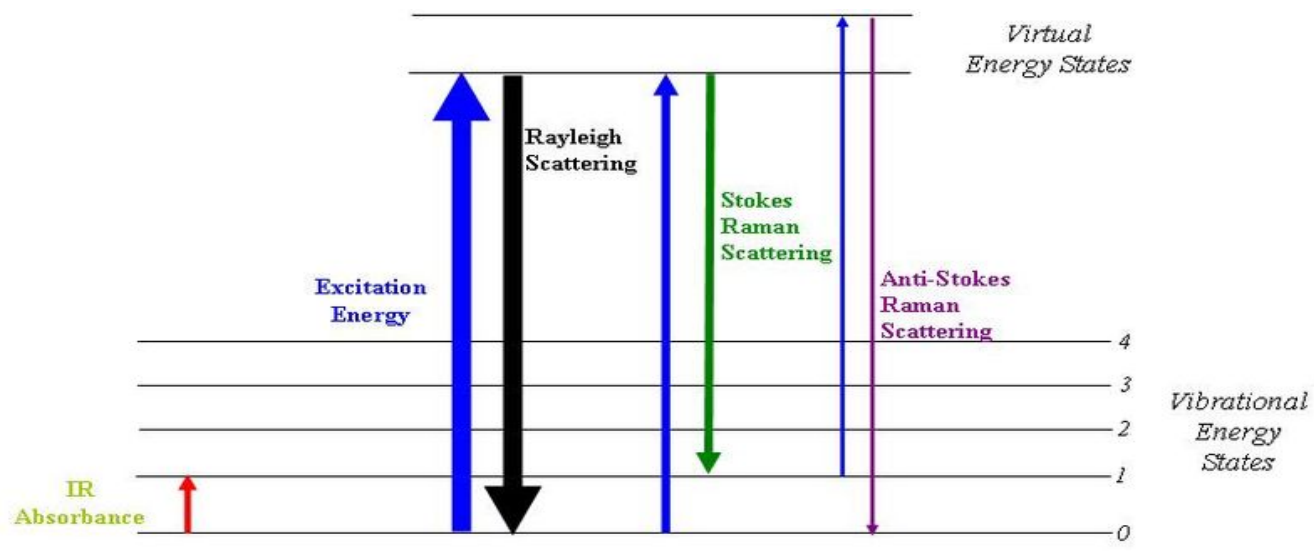

Figure 1.1. Illustration of possible energy transfers during a scattering process between a molecule and an incident photon.

examination of minerals, cells, proteins, and materials such as polymers and ceramics.

However, Raman spectroscopy has its intrinsic deficit in sensitivity. As we mentioned earlier, the probability for a Raman scattering to occur is extremely low. Specifically, the cross section of a Raman scattering is around $10^{-29} \mathrm{~cm}^{2}$, whereas the counterpart of fluorescence is $10^{-19} \mathrm{~cm}^{2}$ [4]. Consequently, Raman spectroscopy would be limited for only the assay on bulk substance if the sensitivity issue can't be fixed. But, fortunately, the emergence of SERS technology has made this deficit history as SERS is capable of enhancing Raman scattering cross section by factors up to $10^{14}$, 
making Raman spectroscopy comparable to fluorescence [5] for ultrasensitive chemical and biological detection at trace amount level.

\subsection{History of SERS}

SERS phenomenon was first observed, though not recognized as such, by Fleischmann et al. [6] in 1974 when they found intense Raman signal, namely, $10^{6}$ enhancement, from pyridine adsorbed on a purposely roughened silver electrode. In fact, their motivation of roughening electrode was to increase the surface area, and, in turn, the number of pyridine molecules trapped on surface. As anticipated, the Raman signal indeed went up, but the magnitude of this increase was too high to agree on their prediction. According to their calculation entirely based on the increased number of trapped molecules, the signal enhancement should not exceed a factor of $10^{2}$. Therefore, a mystery power obviously existed and contributed to that huge Raman signal enhancement.

Jeanmaire and Van Duyne [7] and Albrecht and Creighton [8], independently, were the first recognized that the huge signal enhancement could not be explained simply by the increased number of molecules. In their studies, they slightly roughened the electrode surface and strictly restrained the increased surface area within a factor of 10, but they still obtained signals that had been enhanced far 
more than 10 times. Then, Jeanmaire and Van Duyne proposed an electromagnetic mechanism to explain this phenomenon, on the other hand, Albrecht and Creighton attributed SERS to the resonant Raman scattering from molecular electronic states. As we will go over the detailed description in section 1.3, they both went along the right directions and successfully founded the two primary theories of SERS.

From its inception to the mid 1980s, SERS research had been progressing steadily. To achieve a mechanistic understanding of the signal intensity enhancement, a great deal of effort was put on by condensed matter physicist and chemical physicist. In the end, the majority opinions reached to the conclusion that the enhancement was contributed by two primary components: a long-range electromagnetic effect and a short-range chemical effect [5]. The following ten years became a relatively quiet period as many physics researchers in the field of SERS were replaced by scientists interested in applying it on electrochemistry, biochemistry, catalysis, etc. Over the stretch of last fifteen years, interests of SERS have been picked up again, which is mainly driven by the astonishing discovery of single molecule SERS [9, 10]. In addition, the rapid development of nanoscience and nanotechnology was a key factor in speeding up the research on SERS. At present, the goal of SERS study is to control and manipulate light in nanometer scale in coinage metal thin films or nanostructures, aided 
by the collective electronic excitations or so-called surface plasmons.

\subsection{Mechanisms of SERS}

Two primary theories, electromagnetic enhancement (EM) and chemical enhancement (CHEM), are considered as legitimate explanations for SERS. As implied by their names, the former emphasizes on the amplified electromagnetic fields by the interaction between metals and incident electromagnetic waves, and the latter pays attention to the changes of electronic states, or charge transfers, within molecules induced by the interaction between metals and molecules. These two mechanisms differ substantially from each other in theory, leading to different functions and influences on SERS. On one hand, EM is believed to 1 ) be universal to and present in all SERS cases and 2) to contribute the majority of the total enhancement. On the other hand, CHEM is expected to 1 ) be present only in cases where chemical bonding is formed between molecule and metal, and CHEM 2) produces varying enhancements on molecules depending on their structures and 3 ) has limited contribution to the total enhancement. Details of these mechanisms will be presented as following.

\subsubsection{Electromagnetic enhancement}


When an electromagnetic wave hits on a metal surface, the electric field near metal surface will be different from that in the far field. Particularly, if the surface with roughness or curvature, the wave may excite localized surface plasmon which could significantly amplify electric field at and near the metal surface. The amplification of both the incident field and the scattered field constitutes foundation of EM mechanism and serves as an important model in understanding SERS.

There are many model systems that have been developed to investigate the physics underlying EM. The most famous and simplest one of those is illustrated as a metal sphere placed in an external electric field. The electric field across the sphere is treated as uniform, considering the fact that the wavelength of incident light is far greater than the diameter of spherical particle. Then the electrostatic (Rayleigh) approximation comes in play for this model. By doing so, the induced field at the surface of particle could be expressed as Eq. 1, where

$$
E_{\text {induced }}=\frac{\varepsilon_{1}(\omega)-\varepsilon_{2}}{\varepsilon_{1}(\omega)+2 \varepsilon_{2}} E_{\text {incident }}
$$

$\varepsilon_{1}(\omega)$ is the dielectric constant of metal and a frequency-dependent complex, and $\varepsilon_{2}$ the relative permittivity of ambient phase. Please note that if conditions of a) $\operatorname{Re}\left(\varepsilon_{1}\right)=-2 \varepsilon_{2}$ and $\left.\mathbf{b}\right) \operatorname{Im}\left(\varepsilon_{1}\right)$ being negligible are both satisfied, this function resonates and the induced field will be significantly amplified [11]. The conclusion from this simple model 
could be generalized to any other surface morphologies, though with different numerical factors.

Theoretically, SERS effect may be observed by any metal, but coinage and alkali metals usually provide the strongest enhancement. This could simply be explained as these metals satisfy the resonance conditions at the visible frequencies commonly used in Raman spectroscopy (namely, $532 \mathrm{~nm}, 633 \mathrm{~nm}$, and $785 \mathrm{~nm}$ ). Hence, the principle for SERS materials selection is to find those with $\operatorname{Re}\left(\varepsilon_{1}\right)$ satisfying a resonance condition and $\operatorname{Im}\left(\varepsilon_{1}\right)$ being as small as possible.

In summary, EM accounts for the majority of the total Raman signal enhancement due to the huge resonance of electric field as we discussed above. Moreover, since this mechanism has nothing to do with the analyte, it is expected to produce same effect on whatsoever molecules being studied and be active in any SERS case no matter whether the molecule is chemisorbed or physisorbed to surface. Therefore, most studies on SERS theory have been targeted to this allpurpose mechanism since the inception of SERS [12-25].

\subsubsection{Chemical enhancement}

As indicated, EM should be non-selective for different molecules adsorbed on the same surface, but experimental results say otherwise. Different magnitudes of enhancements have been observed for 
different molecules under exactly same experimental conditions. Furthermore, broad resonances are observed as well in potentialdependent electrochemical experiments [26]. All these showings lead people to believe a second SERS mechanism exists and it works simultaneously in company with EM.

In order to explain above observation, two possible theories related to molecular structure have been proposed. The first one states that the interaction between metal surface and adsorbed molecule shifts and broadens the electronic states of the adsorbate, altering its chemistry. The second one focuses on chemisorptions which lead to new electronic states serving as resonant intermediate states in Raman scattering [27]. For many molecules, the highest occupied molecular orbital (HOMO) and lowest unoccupied molecular orbital (LUMO) are symmetrically disposed in energy level with respect to the Fermi level of the metal (Fig. 1.2). If this is the case, charge transfer either from molecule to metal (arrow 1) or metal to molecule (arrow 2) is apparently more favorable to take place than a direct transfer from HOMO to LUMO (arrow 3). In other words, metal surface facilitates the activity of charge transfer and emissions of characteristic excitations, and this build the foundation of CHEM mechanism. 


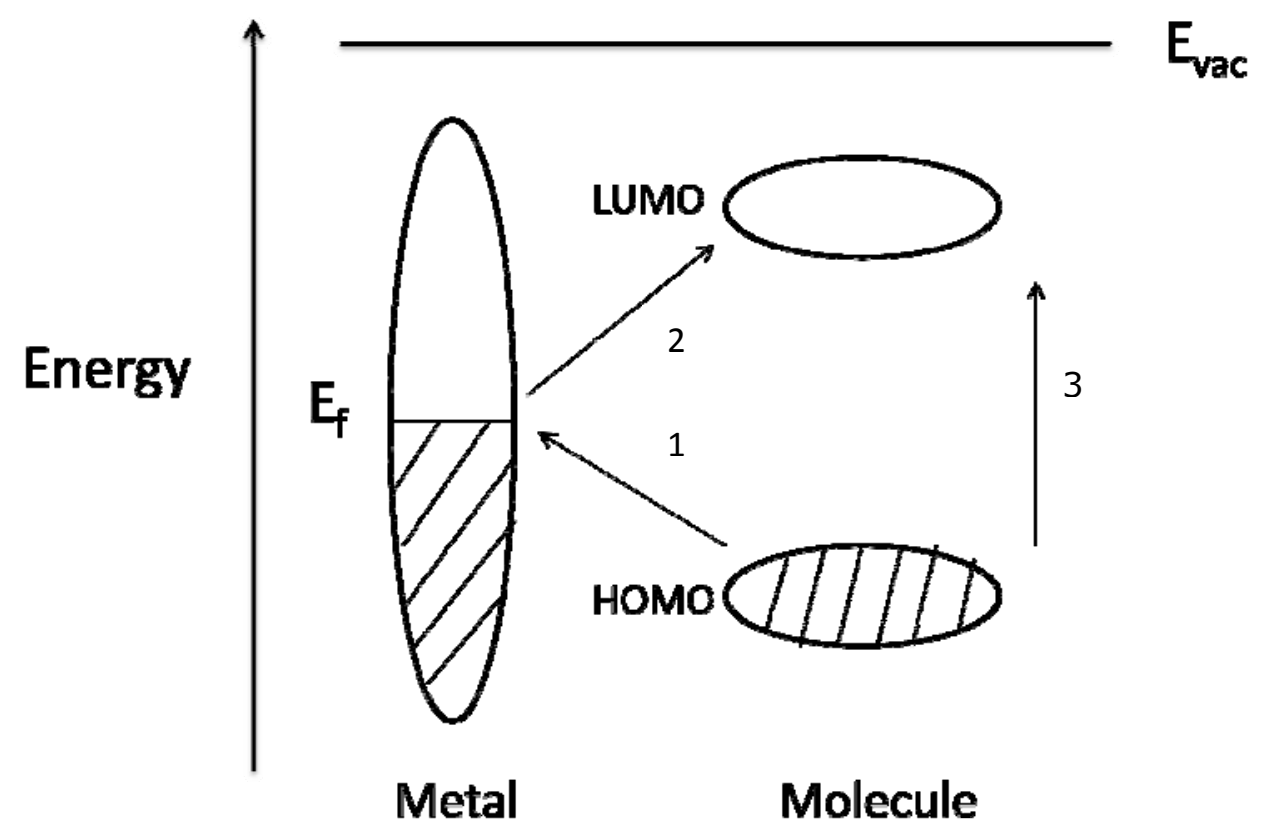

Figure 1.2. Energy level diagram for a molecule adsorbed on metal surface. Possible charge transfer excitations are indicated with arrows.

Regarding to the magnitude, CHEM typically provides one order or two to the total Raman signal enhancement [28]. Usually, study on CHEM is challenging because EM and CHEM are hard to be separated. To solve this issue, Campion et al. carried out a test by using an atomically flat $\mathrm{Cu}$ (111) surface to exclude EM contribution. They ended up with a CHEM factor of 30 in their case [29]. Another feature of CHEM is quite obvious in that it involves interaction with molecules being studied. As a result, the enhancement varies from case to case with different molecules. Furthermore, the molecule has to bond to metal surface in a chemical manner instead of physical in order to induce the enhancement $[26,30]$. Overall, CHEM has limited 
contribution and role in SERS.

\subsection{Enhancement factor of SERS}

Since the discovery of SERS, the magnitude of signal enhancement has always been a focal point in this research topic as it is a direct measure of how well SERS does compared to standard Raman spectroscopy. To quantify it, researchers have introduced enhancement factor (EF), one of the most important SERS parameters, to characterize SERS effect. As Natan [31] pointed out, there are too many different definitions on EF, which causes the concept of EF somehow discrepant and even confusing in literatures. Fortunately, Le Ru et al. [32] summarized all the definitions of EF and put up a nice review on EF. According to their work, the mostly used definition of EF is from the substrate point of view, shown in Eq. 2, where $\mathrm{N}_{\mathrm{SERS}}$ and

$$
E F=\frac{I_{\text {SERS }} / N_{\text {SERS }}}{I_{R S} / N_{R S}}
$$

$\mathrm{N}_{\mathrm{RS}}$ are the average numbers of molecules in scattering volume for SERS and standard Raman spectroscopy measurements, respectively. Same subscript rule applies to intensity (I).

Besides Eq. 2, there is another popular definition of SERS EF that is more relevant to analytical chemistry, specifically, to experimental 
conditions. In this definition, the analytical enhancement factor (AEF) is expressed as Eq. 3, where $\mathrm{C}_{\mathrm{SERS}}$ and $\mathrm{C}_{\mathrm{RS}}$

$$
A E F=\frac{I_{\text {SERS }} / C_{\text {SERS }}}{I_{R S} / C_{R S}}
$$

are the concentrations of analytes for SERS and standard Raman spectroscopy measurements, respectively. $\mathrm{I}_{\mathrm{SERS}}$ and $\mathrm{I}_{\mathrm{RS}}$ are intensities under exactly same experimental conditions, including laser power, acquisition time, and so forth. According to above definitions, AEF (Eq. 3) seems to have an advantage over EF (Eq. 2) because of the simplicity of $A E F$ (Eq. 3). That is, the concentration of analyte is more easily to obtain than the number of molecules per volume. But AEF (Eq. 3) may be essentially inferior to EF (Eq. 2) in accuracy because the concentration of analyte solution does not perfectly relate to the actual number of molecules per volume, given the fact that molecules may distribute unevenly across substrate surface.

\subsection{Fabrications of SERS substrates}

Appropriate metal and correct nanostructure are the two core components to activate SERS effects. Though alkali ( $\mathrm{Li}, \mathrm{Na}$, and K) [33] and transition (Pd, Pt, and $\mathrm{Rh}$ ) [34-36] metals are able to induce SERS effects, coinage metals ( $\mathrm{Au}, \mathrm{Ag}$, and $\mathrm{Cu}$ ) generally are the preferred 
choices for SERS purposes since they are corrosion resistant and their dielectric constants satisfy the resonance condition at Raman excitation wavelengths which are typically near or in visible region [5, 37]. About the correct nanostructure, the requirement is essentially the presence of roughness at nanometer scale. Nanometer scale roughness could be achieved as simply as placing a single nanoparticle on flat surface. Or, in a complex manner, nanometer scale roughness could be created by aggregating a bunch of nanoparticles in the form of a cluster. When it comes to enhancement factor, the latter form is more preferred because it introduces many more junctions and gaps between adjacent nanopartilces. These junctions and gaps are often referred to as SERS "hot spots" [38-42] since relatively strong enhancement effects are usually observed at such sites.

However, the realization of SERS effects is just the first step toward good SERS substrates and does not necessarily guarantee the qualities of SERS substrates. To fabricate reliable SERS substrates with high qualities, many aspects need to be taken into account. In addition to enhancement factor, other important parameters to evaluate SERS substrates for both research and real-world applications include uniformity, repeatability, scalability, shelf life, and cost [43]. In attempting to obtain reliable and scalable SERS substrates, numerous fabrication techniques and approaches have been explored in the past 
pair of decades, including electron beam lithography [44-46], nanosphere lithography $[47,48]$, plating $[49,50]$, laser ablation [51], porous nanoscaffolds [52-54], colloidal aggregation [55], chemical and physical roughening [56-58], oblique angle deposition [59, 60], electrochemical deposition [61], wet chemistry [62], vapor deposition $[63,64]$, de-alloying $[65,66]$, etc. In summary, the present-day techniques for SERS substrates fabrication could be grouped into four categories: template, hybrid, colloid, and direct deposition.

\subsubsection{Template}

The most popular approach for SERS substrate fabrication is template assisted, during which coinage metals are deposited over the templated substrates. Apparently, the key factor of this method lies in the preparation of templates. Electron beam lithography (EBL) is the perfect example to illustrate this fabrication technique as EBL is the most powerful tool to create templates $[44,45,67,68]$. With the help of this sophisticated lithography technique, nearly any possible pattern at nanometer scale could be precisely written on wafer substrates. But the high cost and low throughput of EBL have impeded its wide application. To pursue inexpensive alternatives, Van Duyne et al. developed nanosphere lithography (NSL) [47, 48, 69, 70]. Compared to $E B L, N S L$ replaces resists with polystyrene (PS) nanospheres as the 
pattern or mask materials. Although this method successfully lowers down the manufacturing cost compared to $E B L$, it should be pointed out that the layout and release of nanospheres tends to introduce defects and sacrifice the quality of patterns. Similar rationale, except replacing PS with $\mathrm{SiO}_{2}$, has also been tried out by Jiang et al. [71-74]. The simplest template method is to utilize porous nanosubstrates. In this scenario, porous $\mathrm{Si}[52,54,75,76]$, porous $\mathrm{Al}_{2} \mathrm{O}_{3}[77-84]$ or other porous nanoscaffold $[85,86]$ is prepared by chemical etching and the roughened surfaces will then serve as templates.

\subsubsection{Hybrid}

Hybrid method involves foreign or third party materials aside from coinage metals and base substrates. Specifically, this technique firstly builds bottom layer nanostructures made of foreign materials via various microfabrication techniques and then deposits the coinage metals over the existing nanostructures. Obviously, this method differs from template method in that third party materials are introduced into the systems to provide correct nanostructures. The biggest advantage of hybrid method is its flexibility in selecting desired nanostructures. As long as the nanostructure, no matter what it is made of, is available, hybrid method could make use of it by simply covering the nanostructures with SERS-active metal coatings. Many nanostructures, 
including nanospheres $[87,88]$, nanotubes [89], nanowires [49, 90], nanorods [50, 91-93], etc. have been demonstrated for SERS applications with hybrid method.

\subsubsection{Colloid}

Colloidal Au or Ag is the most commonly used SERS substrate in laboratory research. By adjusting the chemical reaction temperature, time, concentration, $\mathrm{PH}$, and capping agents, various shaped and sized $\mathrm{Au}$ and Ag nanoparticle suspensions have been reported for SERS study [94-97]. The merits of colloidal metals include low cost and high sensitivity, but the shortcomings of this method are quite obvious, too. First, the short shelf life of metal colloid restricts its usage even in laboratory environment, not to mention real-world applications. Second, the large size dispersion has always been an issue for metal colloid, imposing concerns on the repeatability of SERS measurements. Third, capping agents are typically required for metal colloids to remain stable. These organic molecules, directly attached with metal nanoparticles, would cause interfering signals for Raman measurement. All in all, despite the exciting results shown with colloid $\mathrm{Au}$ and $\mathrm{Ag}$ in research community, colloid method is not considered as a mature solution for large scale production and real-world applications of SERS.

\subsubsection{Direct deposition}


In direct deposition, as the name implies, coinage metals are deposited onto wafer substrates without pre-patterning. Oblique angle deposition (OAD) $[59,60,98]$ is a good example to illustrate this philosophy. In OAD, a modified physical vapor deposition technique, the substrate surface normal is positioned at a certain angle to the incoming vapor so that the Ag nanorods could be grown preferentially along a specific direction due to a so-called geometrical shadowing effect. Metal island films serves well as another example of direct deposition. In this method, an ultra thin noble metal film is deposited onto wafer substrates and then the substrates are thermal annealed at mildly high temperature. Due to the high surface free energy, the metal film has a tendency to transform into discontinuous island arrays $[99,100]$. De-alloying is also an example of direct deposition method for SERS substrates fabrication. In this method, a film of $\mathrm{Au}$ and $\mathrm{Ag}$ alloy is firstly deposited onto wafer substrates, and then chemical etching is applied to etch out the Ag from the alloy matrix, leaving a porous Au film on substrates as SERS-active component $[65,66,101$, 102].

\subsection{Applications of SERS}

Owing to its remarkable capability of identifying chemical species and providing structural information at molecular level, SERS has been 
widely employed in a variety of fields including materials science, environmental science, biological engineering, catalysis, homeland security, electrochemistry, food science, forensic science, biomedical diagnostics, etc. In this section, two representative applications of SERS, namely, biosensing and chemical detection, will be briefly reviewed.

\subsubsection{Biosensing}

During the last two decades, interests in detection of biomolecules have grown rapidly by the encouragement of SERS's ultra sensitivity and selectivity. In a typical SERS biosensing process, biomolecules are assembled as a monolayer covering the ensemble of Au or Ag nanoparticles. The Raman spectra are acquired on the basis of population averaged data and have shown good repeatability. Thus far, detection of biomolecules such as glucose [103-105], gene [106, 107], nucleic acid [108], virus [109-111], bacterium [112-115], and biologic toxin [116] have been reported with SERS biosensors.

Particularly, the detection of single biological molecule with SERS has driven lots of interests in the past decade. The concept of single molecule SERS detection was initiated 16 years ago $[9,10]$. To date, SERS method has been used to detect a variety of single molecules, including nucleic acid [117], fluorescent dyes [118, 119], 
neurotransmitter [120], single protein such as cytochrome c [121], horseradish peroxidase [122], myoglobin [123], and hemoglobin [124].

A few extra points need to be pointed out for single molecule SERS. First, single molecule SERS offers great advantages over single fluorescence in that the former provides richer structural information and does not necessarily require labeling. Second, all reported single molecule SERS was observed with aggregated silver nanoparticles, which implies that only SERS "hot spots" are sensitive enough for single molecule detection. Third, this single molecule SERS technique was mostly applied to resonant molecule dyes and hence lacks of versatility to other molecules.

\subsubsection{Chemical detection}

Detection of chemicals at trace amount is of great interests for food safety, environmental protection, and homeland security. Many researchers have demonstrated that SERS has potential to serve for these needs.

In food safety, melamine [125-127] and pesticides [128] in contaminated food have been successfully detected at trace levels. With respect to environmental protection [129], SERS has also been used in monitoring pollutants such as nitrate and nitrite [130], uranium [131], perchlorate [132], nicotine [133], cyanide [134], 
saxitoxin [135] and uranyl ions [136]. As for home land security [137], research has indicated that SERS is able to detect trace amount explosives including half mustard agent [138], dinitrobenzenethiol [139], trinitrotoluene (TNT) and dinitrotuluene (DNT) $[140,141]$.

\subsection{Prospects of SERS}

Despite great efforts has been made to develop SERS technology, there remain critical issues that need to be addressed before the large scale production and practical applications of SERS. For real world applications, SERS substrates firstly need to be fabricated in reliable and inexpensive ways. Secondly, SERS substrate should produce spectral response consistently. To achieve these goals, micro/nanofabrication techniques need to be further developed and refined. The improved techniques should be capable of (1) controlling nanostructures in terms of size, spacing, shape, and geometry, (2) growing uniform nanostructures at large scale, and (3) integrating with conventional fabrication techniques.

Recently, a new direction for SERS research has been proposed by Van Duyne et al. [142]. That is, SERS may benefit significantly from exploring new excitation frequency in the ultraviolet (UV) region where most biological molecules have electronic resonances. To do so, coinage metals might have to be replaced by new group metals in 
order to satisfy Eq. 1. But this small loss could be completely outweighed by the potentially huge gain.

\subsection{Objectives of research}

The objectives of this doctoral study are aimed at two aspects. On one hand, from the perspective of materials growth, this research will focus on the design and fabrication of four plasmonic Au nanostructures, namely, faceted $\mathrm{ZnO} / \mathrm{Au}$ nanonecklace arrays, Au nanoisland arrays, nanoporous $\mathrm{Si} / \mathrm{Au}$ composites and plasma coated SERS-active nanostructures. On the other hand, this research is going to screen aforementioned plasmonic $\mathrm{Au}$ nanostructures and evaluate their potentials for SERS applications. The criteria for this evaluation include repeatability, sensitivity, cost, scalability, shelf life and fabrication complexity. The detailed descriptions of these criteria are explained as follows (in no particular order).

\section{Repeatability}

Whether the SERS substrates produce repeatable spectral response from spot to spot.

\section{Sensitivity}

Whether the SERS substrates demonstrate signal enhancement over standard Raman measurement. Usually characterized with EF.

\section{Cost}


Whether the cost of manufacturing SERS substrates is reasonable in terms of raw materials, tools, and other resources.

\section{Scalability}

Whether the manufacturing of SERS substrates is compatible with standard wafer processing flow.

\section{Shelf life}

Whether the SERS substrates remain unchanged under ambient conditions.

\section{Fabrication complexity}

Whether the fabrication of SERS substrates requires complex procedures.

\subsection{Outline of dissertation}

In this dissertation, we report four types of SERS-active (plasmonic Au) substrates regarding to their fabrication, characterization, SERS testing and optimization, followed by a thorough summary and comparison among these presented plasmonic Au nanostructures. The content of dissertation is organized as follows.

In Chapter 2, faceted $\mathrm{ZnO} / \mathrm{Au}$ nanonecklace arrays epitaxially grown on r-plane sapphire substrates by chemical vapor deposition and sputtering will be introduced.

In Chapter 3, Au nanoisland arrays prepared by repeated 
sputtering and post-deposition annealing will be presented.

In Chapter 4, nanoporous Si/Au composites resulting from metal-assisted wet etching and sputtering will be reported.

In Chapter 5, we will present a novel plasma nanocoating technique that overcoats $\mathrm{SiO}_{2} / \mathrm{Au}$ SERS-active nanostructures with an ultra thin polymer layer, followed by the demonstration of benefits brought by such plasma nanocoating.

In Chapter 6, a summary on these four plasmonic Au nanostructures will be reviewed against each criterion we mentioned in section 1.8. To further improve the SERS performance, corresponding recommendations to each plasmonic nanostructure will be proposed in this chapter as well.

\subsection{Reference}

1. Smith, E. and Dent, G., Morden Raman Spectroscopy: A Practical Approach, 2005, Chichester, UK, Wiely.

2. Long, D., The Raman Effect: A Unified Treatment of the Theory of Raman Scattering by Molecules, 2002, Chichester, UK, Wiely.

3. McCreery, R., Chemical Analysis, Raman Spectroscopy for Chemcial Analysis, 2000, 157, New York, Wiely.

4. Aroca, R., Surface-Enhanced Vibrational Spectrscopy, 2006, Chichester, UK, Wiely.

5. Moskovits, M., Surface-enhanced spectroscopy, Reviews of Modern Physics, 1985, 57, p. 783-826. 
6. Fleischmann, M., Hendra, P. J. and McQuillan, A. J., Raman spectra of pyridine adsorbed at a silver electrode, Chemical Physics Letters, 1974, 26, p. 163-166.

7. Jeanmaire, D. L. and Duyne, R. P. V., Surface raman spectroelectrochemistry: Part I. Heterocyclic, aromatic, and aliphatic amines adsorbed on the anodized silver electrode, Journal of Electroanalytical Chemistry and Interfacial Electrochemistry, 1977, 84, p. 1-20.

8. Albrecht, M. G. and Creighton, J. A., Anomalously intense Raman spectra of pyridine at a silver electrode, Journal of the American Chemical Society, 1977, 99, p. 5215-5217.

9. Kneipp, K., Wang, Y., Kneipp, H., Perelman, L. T., Itzkan, I., Dasari, R. R. and Feld, M. S., Single Molecule Detection Using Surface-Enhanced Raman Scattering (SERS), Physical Review Letters, 1997, 78, p. 1667-1670.

10. Nie, S. and Emory, S. R., Probing Single Molecules and Single Nanoparticles by Surface-Enhanced Raman Scattering, Science, 1997, 275, p. 1102-1106.

11. Campion, A. and Kambhampati, P., Surface-enhanced Raman scattering, Chemical Society Reviews, 1998, 27, p. 241-250.

12. Emory, S. R., Haskins, W. E. and Nie, S., Direct Observation of SizeDependent Optical Enhancement in Single Metal Nanoparticles, Journal of the American Chemical Society, 1998, 120, p. 8009-8010.

13. Kerker, M., Electromagnetic model for surface-enhanced Raman scattering (SERS) on metal colloids, Accounts of Chemical Research, 1984, 17, p. 271277.

14. Metiu, H. and Das, P., The Electromagnetic Theory of Surface Enhanced Spectroscopy, Annual Review of Physical Chemistry, 1984, 35, p. 507-536.

15. Zeman, E. J. and Schatz, G. C., An accurate electromagnetic theory study of surface enhancement factors for silver, gold, copper, lithium, sodium, aluminum, gallium, indium, zinc, and cadmium, The Journal of Physical Chemistry, 1987, 91, p. 634-643.

16. Haynes, C. L. and Van Duyne, R. P., Plasmon-Sampled Surface-Enhanced Raman Excitation Spectroscopyt, Journal of Physical Chemistry B, 2003, 107, p. 7426-7433. 
17. McFarland, A. D., Young, M. A., Dieringer, J. A. and Van Duyne, R. P., Wavelength-Scanned Surface-Enhanced Raman Excitation Spectroscopy, The Journal of Physical Chemistry B, 2005, 109, p. 11279-11285.

18. Dieringer, J. A., McFarland, A. D., Shah, N. C., Stuart, D. A., Whitney, A. V., Yonzon, C. R., Young, M. A., Zhang, X. and Van Duyne, R. P., Introductory Lecture Surface enhanced Raman spectroscopy: new materials, concepts, characterization tools, and applications, Faraday Discussions, 2006, 132, p. 9-26.

19. Kennedy, B. J., Spaeth, S., Dickey, M. and Carron, K. T., Determination of the Distance Dependence and Experimental Effects for Modified SERS Substrates Based on Self-Assembled Monolayers Formed Using Alkanethiols, The Journal of Physical Chemistry B, 1999, 103, p. 3640-3646.

20. Yang, W.-H., Schatz, G. C. and Van Duyne, R. P., Discrete dipole approximation for calculating extinction and Raman intensities for small particles with arbitrary shapes, The Journal of Chemical Physics, 1995, 103, p. 869-875.

21. Kelly, K. L., Coronado, E., Zhao, L. L. and Schatz, G. C., The Optical Properties of Metal Nanoparticles: The Influence of Size, Shape, and Dielectric Environment, The Journal of Physical Chemistry B, 2002, 107, p. 668-677.

22. Link, S. and El-Sayed, M. A., Spectral Properties and Relaxation Dynamics of Surface Plasmon Electronic Oscillations in Gold and Silver Nanodots and Nanorods, The Journal of Physical Chemistry B, 1999, 103, p. 8410-8426.

23. Wang, D. S. and Kerker, M., Enhanced Raman scattering by molecules adsorbed at the surface of colloidal spheroids, Physical Review B, 1981, 24, p. 1777-1790.

24. Blatchford, C. G., Campbell, J. R. and Creighton, J. A., Plasma resonance enhanced raman scattering by absorbates on gold colloids: The effects of aggregation, Surface Science, 1982, 120, p. 435-455.

25. Felidj, N., Aubard, J., Levi, G., Krenn, J. R., Hohenau, A., Schider, G., Leitner, A. and Aussenegg, F. R., Optimized surface-enhanced Raman scattering on gold nanoparticle arrays, Applied Physics Letters, 2003, 82, p. 3095-3097.

26. Lombardi, J. R., Birke, R. L., Lu, T. and Xu, J., Charge-Transfer Theory of Surface Enhanced Raman Spectroscopy: Herzberg-Teller Contributions, Journal of Chemical Physics, 1986, 84, p. 4174-4180. 
27. Kneipp, K., Kneipp, H., Itzkan, I., Dasari, R. R. and Feld, M. S., Ultrasensitive Chemical Analysis by Raman Spectroscopy, Chemical Reviews, 1999, 99, p. 2957-2976.

28. Kambhampati, P., Child, C. M., Foster, M. C. and Campion, A., On the chemical mechanism of surface enhanced Raman scattering: Experiment and theory, The Journal of Chemical Physics, 1998, 108, p. 5013-5026.

29. Campion, A., Ivanecky, J. E., Child, C. M. and Foster, M., On the Mechanism of Chemical Enhancement in Surface-Enhanced Raman Scattering, Journal of the American Chemical Society, 1995, 117, p. 11807-11808.

30. Lombardi, J. R. and Birke, R. L., A Unified Approach to Surface-Enhanced Raman Spectroscopy, The Journal of Physical Chemistry C, 2008, 112, p. 5605-5617.

31. Natan, M. J., Concluding Remarks Surface enhanced Raman scattering, Faraday Discussions, 2006, 132, p. 321-328.

32. Le Ru, E. C., Blackie, E., Meyer, M. and Etchegoin, P. G., Surface Enhanced Raman Scattering Enhancement Factors: A Comprehensive Study, The Journal of Physical Chemistry C, 2007, 111, p. 13794-13803.

33. Schulze, W., Breithaupt, B., Charlé, K. P. and Kloss, U., Surface Enhanced Raman Scattering (SERS) at Matrix Isolated Metal Clusters of Ag, $\mathrm{Na}$ and $\mathrm{K}$, Berichte der Bunsengesellschaft für physikalische Chemie, 1984, 88, p. 308310.

34. Abdelsalam, M. E., Mahajan, S., Bartlett, P. N., Baumberg, J. J. and Russell, A. E., SERS at Structured Palladium and Platinum Surfaces, Journal of the American Chemical Society, 2007, 129, p. 7399-7406.

35. Mrozek, M. F., Xie, Y. and Weaver, M. J., Surface-Enhanced Raman Scattering on Uniform Platinum-Group Overlayers: Preparation by Redox Replacement of Underpotential-Deposited Metals on Gold, Anal Chem, 2001, 73, p. 59535960.

36. Tian, Z.-Q., Yang, Z.-L., Ren, B., Li, J.-F., Zhang, Y., Lin, X.-F., Hu, J.-W. and Wu, D.-Y., Surface-enhanced Raman scattering from transition metals with special surface morphology and nanoparticle shape, Faraday Discussions, 2006, 132, p. 159-170. 
37. Johnson, P. B. and Christy, R. W., Optical Constants of the Noble Metals, Physical Review B, 1972, 6, p. 4370-4379.

38. Moskovits, M., Surface-enhanced Raman spectroscopy: a brief retrospective, Journal of Raman Spectroscopy, 2005, 36, p. 485-496.

39. Futamata, M., Single molecule sensitivity in SERS: importance of junction of adjacent Ag nanoparticles, Faraday Discussions, 2006, 132, p. 45-61.

40. Svedberg, F., Li, Z., Xu, H. and Käll, M., Creating Hot Nanoparticle Pairs for Surface-Enhanced Raman Spectroscopy through Optical Manipulation, Nano Letters, 2006, 6, p. 2639-2641.

41. Camden, J. P., Dieringer, J. A., Wang, Y., Masiello, D. J., Marks, L. D., Schatz, G. C. and Van Duyne, R. P., Probing the Structure of Single-Molecule SurfaceEnhanced Raman Scattering Hot Spots, Journal of the American Chemical Society, 2008, 130, p. 12616-12617.

42. Maher, R. C., Maier, S. A., Cohen, L. F., Koh, L., Laromaine, A., Dick, J. A. G. and Stevens, M. M., Exploiting SERS Hot Spots for Disease-Specific Enzyme Detectiont, The Journal of Physical Chemistry C, 2009, 114, p. 7231-7235.

43. Tripp, R. A., Dluhy, R. A. and Zhao, Y., Novel nanostructures for SERS biosensing, Nano Today, 2008, 3, p. 31-37.

44. Sackmann, M., Bom, S., Balster, T. and Materny, A., Nanostructured gold surfaces as reproducible substrates for surface-enhanced Raman spectroscopy, Journal of Raman Spectroscopy, 2007, 38, p. 277-282.

45. Billot, L., Lamy de la Chapelle, M., Grimault, A. S., Vial, A., Barchiesi, D., Bijeon, J. L., Adam, P. M. and Royer, P., Surface enhanced Raman scattering on gold nanowire arrays: Evidence of strong multipolar surface plasmon resonance enhancement, Chemical Physics Letters, 2006, 422, p. 303-307.

46. Yu, Q., Guan, P., Qin, D., Golden, G. and Wallace, P. M., Inverted SizeDependence of Surface-Enhanced Raman Scattering on Gold Nanohole and Nanodisk Arrays, Nano Letters, 2008, 8, p. 1923-1928.

47. Jensen, T. R., Malinsky, M. D., Haynes, C. L. and Van Duyne, R. P., Nanosphere Lithography: Tunable Localized Surface Plasmon Resonance Spectra of Silver Nanoparticles, Journal of Physical Chemistry B, 2000, 104, p. 10549-10556. 
48. Ormonde, A. D., Hicks, E. C. M., Castillo, J. and Van Duyne, R. P., Nanosphere Lithography: Fabrication of Large-Area Ag Nanoparticle Arrays by Convective Self-Assembly and Their Characterization by Scanning UV-Visible Extinction Spectroscopy, Langmuir, 2004, 20, p. 6927-6931.

49. Khan, M. A., Hogan, T. P. and Shanker, B., Gold-coated zinc oxide nanowirebased substrate for surface-enhanced Raman spectroscopy, Journal of Raman Spectroscopy, 2009, 40, p. 1539-1545.

50. Cheng, C., Yan, B., Wong, S. M., Li, X., Zhou, W., Yu, T., Shen, Z., Yu, H. and Fan, H. J., Fabrication and SERS Performance of Silver-NanoparticleDecorated Si/ZnO Nanotrees in Ordered Arrays, ACS Applied Materials \& Interfaces, 2010, 2, p. 1824-1828.

51. Neddersen, J., Chumanov, G. and Cotton, T. M., Laser Ablation of Metals: $A$ New Method for Preparing SERS Active Colloids, Applied Spectroscopy, 1993, 47, p. 1959-1964.

52. Lin, H., Mock, J., Smith, D., Gao, T. and Sailor, M. J., Surface-Enhanced Raman Scattering from Silver-Plated Porous Silicon, Journal of Physical Chemistry B, 2004, 108, p. 11654-11659.

53. Walsh, R. J. and Chumanov, G., Silver Coated Porous Alumina as a New Substrate for Surface-Enhanced Raman Scattering, Applied Spectroscopy, 2001, 55, p. 1695-1700.

54. Myeong-Lok, S., Sung-Jin, C., David, J. B., Tae Jung, P., Jae-Hyuk, A., Sang Yup, L. and Yang-Kyu, C., A nanoforest structure for practical surfaceenhanced Raman scattering substrates, Nanotechnology, 2012, 23, p. 095301.

55. Jana, N. R. and Pal, T., Anisotropic Metal Nanoparticles for Use as SurfaceEnhanced Raman Substrates, Advanced Materials, 2007, 19, p. 1761-1765.

56. Williamson, T. L., Guo, X., Zukoski, A., Sood, A., Díaz, D. J. and Bohn, P. W., Porous GaN as a Template to Produce Surface-Enhanced Raman ScatteringActive Surfaces, Journal of Physical Chemistry B, 2005, 109, p. 20186-20191.

57. Christou, K., Knorr, I., Ihlemann, J. r., Wackerbarth, H. and Beushausen, V., Fabrication and Characterization of Homogeneous Surface-Enhanced Raman Scattering Substrates by Single Pulse UV-Laser Treatment of Gold and Silver Films, Langmuir, 2010, 26, p. 18564-18569. 
58. Xue, G., Dong, J. and Zhang, M., Surface-enhanced Raman scattering (SERS) and surface-enhanced resonance Raman scattering (SERRS) on HNO3roughened copper foil, Appl. Spectrosc., 1991, 45, p. 756-759.

59. Chaney, S. B., Shanmukh, S., Dluhy, R. A. and Zhao, Y. P., Aligned silver nanorod arrays produce high sensitivity surface-enhanced Raman spectroscopy substrates, Applied Physics Letters, 2005, 87, p. 031908-3.

60. Liu, Y., Fan, J., Zhao, Y. P., Shanmukh, S. and Dluhy, R. A., Angle dependent surface enhanced Raman scattering obtained from a $\mathrm{Ag}$ nanorod array substrate, Applied Physics Letters, 2006, 89, p. 173134-3.

61. He, P., Liu, H., Li, Z., Liu, Y., Xu, X. and Li, J., Electrochemical Deposition of Silver in Room-Temperature Ionic Liquids and Its Surface-Enhanced Raman Scattering Effect, Langmuir, 2004, 20, p. 10260-10267.

62. Ahern, A. M. and Garrell, R. L., In situ photoreduced silver nitrate as a substrate for surface-enhanced Raman spectroscopy, Anal Chem, 1987, 59, p. 2813-2816.

63. Semin, D. J. and Rowlen, K. L., Influence of vapor deposition parameters on SERS active Ag film morphology and optical properties, Anal Chem, 1994, 66, p. 4324-4331.

64. Roark, S. E. and Rowlen, K. L., Thin silver films: influence of substrate and postdeposition treatment on morphology and optical properties, Anal Chem, 1994,66 , p. 261-270.

65. Erlebacher, J., Aziz, M. J., Karma, A., Dimitrov, N. and Sieradzki, K., Evolution of nanoporosity in dealloying, Nature, 2001, 410, p. 450-453.

66. Biener, J., Nyce, G. W., Hodge, A. M., Biener, M. M., Hamza, A. V. and Maier, S. A., Nanoporous Plasmonic Metamaterials, Advanced Materials, 2008, 20, p. 1211-1217.

67. Reilly, T. H., Corbman, J. D. and Rowlen, K. L., Vapor Deposition Method for Sensitivity Studies on Engineered Surface-Enhanced Raman Scattering-Active Substrates, Anal Chem, 2007, 79, p. 5078-5081.

68. De, J., S, M. A., Giesfeldt, K. S., Oran, J. M., Abu-Hatab, N. A., Lavrik, N. V. and Sepaniak, M. J., Nanofabrication of Densely Packed MetalPolymer Arrays for Surface-Enhanced Raman Spectrometry, Applied Spectroscopy, 2005, 59, p. $1501-1508$. 
69. Haynes, C. L. and Van Duyne, R. P., Nanosphere Lithography: A Versatile Nanofabrication Tool for Studies of Size-Dependent Nanoparticle Optics, The Journal of Physical Chemistry B, 2001, 105, p. 5599-5611.

70. Hulteen, J. C., Treichel, D. A., Smith, M. T., Duval, M. L., Jensen, T. R. and Van Duyne, R. P., Nanosphere Lithography: Size-Tunable Silver Nanoparticle and Surface Cluster Arrays, The Journal of Physical Chemistry B, 1999, 103, p. 3854-3863.

71. Jiang, P., Large-Scale Fabrication of Periodic Nanostructured Materials by Using Hexagonal Non-Close-Packed Colloidal Crystals as Templates, Langmuir, 2006, 22, p. 3955-3958.

72. Jiang, P. and McFarland, M. J., Large-Scale Fabrication of Wafer-Size Colloidal Crystals, Macroporous Polymers and Nanocomposites by Spin-Coating, Journal of the American Chemical Society, 2004, 126, p. 13778-13786.

73. Lin, T.-H., Linn, N. C., Tarajano, L., Jiang, B. and Jiang, P., Electrochemical SERS at Periodic Metallic Nanopyramid Arrays, The Journal of Physical Chemistry C, 2009, 113, p. 1367-1372.

74. Liu, X., Sun, C.-H., Linn, N. C., Jiang, B. and Jiang, P., Wafer-Scale SurfaceEnhanced Raman Scattering Substrates with Highly Reproducible Enhancement, The Journal of Physical Chemistry C, 2009, 113, p. 1480414811.

75. Chan, S., Kwon, S., Koo, T. W., Lee, L. P. and Berlin, A. A., Surface-Enhanced Raman Scattering of Small Molecules from Silver-Coated Silicon Nanopores, Advanced Materials, 2003, 15, p. 1595-1598.

76. Chattopadhyay, S., Lo, H.-C., Hsu, C.-H., Chen, L.-C. and Chen, K.-H., Surface-Enhanced Raman Spectroscopy Using Self-Assembled Silver Nanoparticles on Silicon Nanotips, Chemistry of Materials, 2005, 17, p. 553559.

77. Walsh, R. J. and Chumanov, G., Silver Coated Porous Alumina as a New Substrate for Surface-Enhanced Raman Scattering, Applied Spectroscopy, 2001, 55, p. 1695-1700.

78. Kartopu, G., Es-Souni, M., Sapelkin, A. V. and Dunstan, D., A novel SERSactive substrate system: Template-grown nanodot-film structures, physica status solidi (a), 2006, 203, p. R82-R84. 
79. Broglin, B. L., Andreu, A., Dhussa, N., Heath, J. A., Gerst, J., Dudley, B., Holland, D. and El-Kouedi, M., Investigation of the Effects of the Local Environment on the Surface-Enhanced Raman Spectra of Striped Gold/Silver Nanorod Arrays, Langmuir, 2007, 23, p. 4563-4568.

80. Gu, G. H., Kim, J., Kim, L. and Suh, J. S., Optimum Length of Silver Nanorods for Fabrication of Hot Spots, The Journal of Physical Chemistry C, 2007, 111, p. 7906-7909.

81. Lombardi, I., Cavallotti, P. L., Carraro, C. and Maboudian, R., Template assisted deposition of Ag nanoparticle arrays for surface-enhanced Raman scattering applications, Sensors and Actuators, B: Chemical, 2007, 125, p. 353-356.

82. Schierhorn, M., Lee, S. J., Boettcher, S. W., Stucky, G. D. and Moskovits, M., Metal-Silica Hybrid Nanostructures for Surface-Enhanced Raman Spectroscopy, Advanced Materials, 2006, 18, p. 2829-2832.

83. Du, Y., Shi, L., He, T., Sun, X. and Mo, Y., SERS enhancement dependence on the diameter and aspect ratio of silver-nanowire array fabricated by anodic aluminium oxide template, Applied Surface Science, 2008, 255, p. 1901-1905.

84. Wang, H. H., Liu, C. Y., Wu, S. B., Liu, N. W., Peng, C. Y., Chan, T. H., Hsu, C. F., Wang, J. K. and Wang, Y. L., Highly Raman-Enhancing Substrates Based on Silver Nanoparticle Arrays with Tunable Sub-10 nm Gaps, Advanced Materials, 2006, 18, p. 491-495.

85. Henley, S. J., Carey, J. D. and Silva, S. R. P., Silver-nanoparticle-decorated carbon nanoscaffolds: Application as a sensing platform, Applied Physics Letters, 2006, 89, p. 183120.

86. Sun, Y., Liu, K., Miao, J., Wang, Z., Tian, B., Zhang, L., Li, Q., Fan, S. and Jiang, K., Highly Sensitive Surface-Enhanced Raman Scattering Substrate Made from Superaligned Carbon Nanotubes, Nano Letters, 2010, 10, p. 17471753.

87. Dick, L. A., McFarland, A. D., Haynes, C. L. and Van Duyne, R. P., Metal Film over Nanosphere (MFON) Electrodes for Surface-Enhanced Raman Spectroscopy (SERS): Improvements in Surface Nanostructure Stability and Suppression of Irreversible Loss, The Journal of Physical Chemistry B, 2001, 106, p. 853-860.

88. Bao, L.-L., Mahurin, S. M., Liang, C.-D. and Dai, S., Study of silver films over silica beads as a surface-enhanced Raman scattering (SERS) substrate for 
detection of benzoic acid, Journal of Raman Spectroscopy, 2003, 34, p. 394398.

89. Li, X., Chen, G., Yang, L., Jin, Z. and Liu, J., Multifunctional Au-Coated TiO2 Nanotube Arrays as Recyclable SERS Substrates for Multifold Organic Pollutants Detection, Advanced Functional Materials, 2010, 20, p. 2815-2824.

90. Deng, S., Fan, H. M., Zhang, X., Loh, K. P., Cheng, C. L., Sow, C. H. and Foo, Y. L., An effective surface-enhanced Raman scattering template based on a Ag nanocluster-ZnO nanowire array, Nanotechnology, 2009, 20, p. 175705.

91. Tatsunori, S., Yuto, T., Ryo, N., Nikolay, N. N., Petar, A. A., Toshiharu, S. and Minoru, O., Surface enhanced Raman scattering properties using Au-coated ZnO nanorods grown by two-step, off-axis pulsed laser deposition, Journal of Physics D: Applied Physics, 2008, 41, p. 235304.

92. Chen, L., Luo, L., Chen, Z., Zhang, M., Zapien, J. A., Lee, C. S. and Lee, S. T., ZnO/Au Composite Nanoarrays As Substrates for Surface-Enhanced Raman Scattering Detection, The Journal of Physical Chemistry C, 2009, 114, p. 93100.

93. Song, W., Han, X., Chen, L., Yang, Y., Tang, B., Ji, W., Ruan, W., Xu, W., Zhao, B. and Ozaki, Y., Site-specific deposition of Ag nanoparticles on ZnO nanorod arrays via galvanic reduction and their SERS applications, Journal of Raman Spectroscopy, 2010, 41, p. 907-913.

94. Rivas, L., Sanchez-Cortes, S., García-Ramos, J. V. and Morcillo, G., Mixed Silver/Gold Colloids: A Study of Their Formation, Morphology, and SurfaceEnhanced Raman Activity, Langmuir, 2000, 16, p. 9722-9728.

95. Nickel, U., zu Castell, A., Pöppl, K. and Schneider, S., A Silver Colloid Produced by Reduction with Hydrazine as Support for Highly Sensitive Surface-Enhanced Raman Spectroscopyt, Langmuir, 2000, 16, p. 9087-9091.

96. Rycenga, M., Hou, K. K., Cobley, C. M., Schwartz, A. G., Camargo, P. H. C. and Xia, Y., Probing the surface-enhanced Raman scattering properties of AuAg nanocages at two different excitation wavelengths, Physical Chemistry Chemical Physics, 2009, 11, p. 5903-5908.

97. Rycenga, M., Cobley, C. M., Zeng, J., Li, W., Moran, C. H., Zhang, Q., Qin, D. and Xia, Y., Controlling the Synthesis and Assembly of Silver Nanostructures for Plasmonic Applications, Chemical Reviews, 2011, 111, p. 3669-3712. 
98. Driskell, J. D., Shanmukh, S., Liu, Y., Chaney, S. B., Tang, X. J., Zhao, Y. P. and Dluhy, R. A., The Use of Aligned Silver Nanorod Arrays Prepared by Oblique Angle Deposition as Surface Enhanced Raman Scattering Substrates, The Journal of Physical Chemistry C, 2008, 112, p. 895-901.

99. Xin, S. and Hao, L., Gold nanoisland arrays by repeated deposition and postdeposition annealing for surface-enhanced Raman spectroscopy, Nanotechnology, 2013, 24, p. 355706.

100. Chursanova, M., Dzhagan, V., Yukhymchuk, V., Lytvyn, O., Valakh, M., Khodasevich, I., Lehmann, D., Zahn, D., Waurisch, C. and Hickey, S., Nanostructured Silver Substrates With Stable and Universal SERS Properties: Application to Organic Molecules and Semiconductor Nanoparticles, Nanoscale Research Letters, 2009, 5, p. 403 - 409.

101. Ding, Y., Kim, Y. J. and Erlebacher, J., Nanoporous Gold Leaf: "Ancient Technology"/Advanced Material, Advanced Materials, 2004, 16, p. 1897-1900.

102. Ciesielski, P. N., Scott, A. M., Faulkner, C. J., Berron, B. J., Cliffel, D. E. and Jennings, G. K., Functionalized Nanoporous Gold Leaf Electrode Films for the Immobilization of Photosystem I, ACS Nano, 2008, 2, p. 2465-2472.

103. Ma, K., Yuen, J. M., Shah, N. C., Walsh, J. T., Glucksberg, M. R. and Van Duyne, R. P., In Vivo, Transcutaneous Glucose Sensing Using SurfaceEnhanced Spatially Offset Raman Spectroscopy: Multiple Rats, Improved Hypoglycemic Accuracy, Low Incident Power, and Continuous Monitoring for Greater than 17 Days, Anal Chem, 2011, 83, p. 9146-9152.

104. Yuen, J. M., Shah, N. C., Walsh, J. T., Glucksberg, M. R. and Van Duyne, R. P., Transcutaneous Glucose Sensing by Surface-Enhanced Spatially Offset Raman Spectroscopy in a Rat Model, Anal Chem, 2010, 82, p. 8382-8385.

105. Yonzon, C. R., Haynes, C. L., Zhang, X., Walsh, J. T. and Van Duyne, R. P., A Glucose Biosensor Based on Surface-Enhanced Raman Scattering: Improved Partition Layer, Temporal Stability, Reversibility, and Resistance to Serum Protein Interference, Anal Chem, 2003, 76, p. 78-85.

106. Vo-Dinh, T., Allain, L. R. and Stokes, D. L., Cancer gene detection using surface-enhanced Raman scattering (SERS), Journal of Raman Spectroscopy, 2002, 33, p. 511-516.

107. Allain, L. R. and Vo-Dinh, T., Surface-enhanced Raman scattering detection of the breast cancer susceptibility gene BRCA1 using a silver-coated microarray platform, Analytica Chimica Acta, 2002, 469, p. 149-154. 
108. Cao, Y. C., Jin, R. and Mirkin, C. A., Nanoparticles with Raman Spectroscopic Fingerprints for DNA and RNA Detection, Science, 2002, 297, p. 1536-1540.

109. Shanmukh, S., Jones, L., Driskell, J., Zhao, Y., Dluhy, R. and Tripp, R. A., Rapid and Sensitive Detection of Respiratory Virus Molecular Signatures Using a Silver Nanorod Array SERS Substrate, Nano Letters, 2006, 6, p. 2630-2636.

110. Driskell, J. D., Kwarta, K. M., Lipert, R. J., Porter, M. D., Neill, J. D. and Ridpath, J. F., Low-Level Detection of Viral Pathogens by a Surface-Enhanced Raman Scattering Based Immunoassay, Anal Chem, 2005, 77, p. 6147-6154.

111. Isola, N. R., Stokes, D. L. and Vo-Dinh, T., Surface-Enhanced Raman Gene Probe for HIV Detection, Anal Chem, 1998, 70, p. 1352-1356.

112. Goeller, L. J. and Riley, M. R., Discrimination of Bacteria and Bacteriophages by Raman Spectroscopy and Surface-Enhanced Raman Spectroscopy, Applied Spectroscopy, 2007, 61, p. 679-685.

113. Sengupta, A., Laucks, M. L. and Davis, E. J., Surface-Enhanced Raman Spectroscopy of Bacteria and Pollen, Applied Spectroscopy, 2005, 59, p. 1016-1023.

114. Naja, G., Bouvrette, P., Hrapovic, S. and Luong, J. H. T., Raman-based detection of bacteria using silver nanoparticles conjugated with antibodies, Analyst, 2007, 132, p. 679-686.

115. Sengupta, A., Mujacic, M. and Davis, E., Detection of bacteria by surfaceenhanced Raman spectroscopy, Analytical and Bioanalytical Chemistry, 2006, 386, p. $1379-1386$.

116. Grow, A. E., Wood, L. L., Claycomb, J. L. and Thompson, P. A., New biochip technology for label-free detection of pathogens and their toxins, Journal of Microbiological Methods, 2003, 53, p. 221-233.

117. Graham, D., Mallinder, B. J. and Smith, W. E., Surface-Enhanced Resonance Raman Scattering as a Novel Method of DNA Discrimination, Angewandte Chemie International Edition, 2000, 39, p. 1061-1063.

118. Kneipp, K., Kneipp, H., Deinum, G., Itzkan, I., Dasari, R. R. and Feld, M. S., Single-Molecule Detection of a Cyanine Dye in Silver Colloidal Solution Using Near-Infrared Surface-Enhanced Raman Scattering, Applied Spectroscopy, 1998, 52, p. 175-178. 
119. Etchegoin, P., Maher, R. C., Cohen, L. F., Hartigan, H., Brown, R. J. C., Milton, M. J. T. and Gallop, J. C., New limits in ultrasensitive trace detection by surface enhanced Raman scattering (SERS), Chemical Physics Letters, 2003, 375, p. 84-90.

120. Lee, N. S., Hsieh, Y. Z., Paisley, R. F. and Morris, M. D., Surface-enhanced Raman spectroscopy of the catecholamine neurotransmitters and related compounds, Anal Chem, 1988, 60, p. 442-446.

121. Delfino, I., Bizzarri, A. R. and Cannistraro, S., Single-molecule detection of yeast cytochrome c by Surface-Enhanced Raman Spectroscopy, Biophysical Chemistry, 2005, 113, p. 41-51.

122. Bjerneld, E. J., Földes-Papp, Z., Käll, M. and Rigler, R., Single-Molecule Surface-Enhanced Raman and Fluorescence Correlation Spectroscopy of Horseradish Peroxidase, The Journal of Physical Chemistry B, 2002, 106, p. 1213-1218.

123. Bizzarri, A. R. and Cannistraro, S., Surface-Enhanced Resonance Raman Spectroscopy Signals from Single Myoglobin Molecules, Applied Spectroscopy, 2002, 56, p. 1531-1537.

124. Xu, H., Bjerneld, E. J., Käll, M. and Börjesson, L., Spectroscopy of Single Hemoglobin Molecules by Surface Enhanced Raman Scattering, Physical Review Letters, 1999, 83, p. 4357-4360.

125. He, L., Liu, Y., Lin, M., Awika, J., Ledoux, D., Li, H. and Mustapha, A., A new approach to measure melamine, cyanuric acid, and melamine cyanurate using surface enhanced Raman spectroscopy coupled with gold nanosubstrates, Sensing and Instrumentation for Food Quality and Safety, 2008, 2, p. 66-71.

126. Liu, B., Lin, M. and Li, H., Potential of SERS for rapid detection of melamine and cyanuric acid extracted from milk, Sensing and Instrumentation for Food Quality and Safety, 2010, 4, p. 13-19.

127. Zhang, X.-F., Zou, M.-Q., Qi, X.-H., Liu, F., Zhu, X.-H. and Zhao, B.-H., Detection of melamine in liquid milk using surface-enhanced Raman scattering spectroscopy, Journal of Raman Spectroscopy, 2010, 41, p. 16551660.

128. Sánchez-Cortés, S., Domingo, C., García-Ramos, J. V. and Aznárez, J. A., Surface-Enhanced Vibrational Study (SEIR and SERS) of Dithiocarbamate Pesticides on Gold Films, Langmuir, 2001, 17, p. 1157-1162. 
129. Halvorson, R. A. and Vikesland, P. J., Surface-Enhanced Raman Spectroscopy (SERS) for Environmental Analyses, Environmental Science \& Technology, 2010, 44, p. 7749-7755.

130. Ianoul, A., Coleman, T. and Asher, S. A., UV Resonance Raman Spectroscopic Detection of Nitrate and Nitrite in Wastewater Treatment Processes, Anal Chem, 2002, 74, p. 1458-1461.

131. Ruan, C., Luo, W., Wang, W. and Gu, B., Surface-enhanced Raman spectroscopy for uranium detection and analysis in environmental samples, Analytica Chimica Acta, 2007, 605, p. 80-86.

132. Gu, B., Tio, J., Wang, W., Ku, Y.-K. and Dai, S., Raman Spectroscopic Detection for Perchlorate at Low Concentrations, Applied Spectroscopy, 2004, 58, p. 741-744.

133. Bell, S. E. J. and Sirimuthu, N. M. S., Quantitative surface-enhanced Raman spectroscopy, Chemical Society Reviews, 2008, 37, p. 1012-1024.

134. Senapati, D., Dasary, S. S. R., Singh, A. K., Senapati, T., Yu, H. and Ray, P. C., A Label-Free Gold-Nanoparticle-Based SERS Assay for Direct Cyanide Detection at the Parts-per-Trillion Level, Chemistry - A European Journal, 2011, 17, p. 8445-8451.

135. Pearman, W. F., Angel, S. M., Ferry, J. L. and Hall, S., Characterization of the Ag Mediated Surface-Enhanced Raman Spectroscopy of Saxitoxin, Applied Spectroscopy, 2008, 62, p. 727-732.

136. Bao, L., Mahurin, S. M., Haire, R. G. and Dai, S., Silver-Doped Sol-Gel Film as a Surface-Enhanced Raman Scattering Substrate for Detection of Uranyl and Neptunyl Ions, Anal Chem, 2003, 75, p. 6614-6620.

137. Golightly, R. S., Doering, W. E. and Natan, M. J., Surface-Enhanced Raman Spectroscopy and Homeland Security: A Perfect Match?, ACS Nano, 2009, 3, p. 2859-2869.

138. Stuart, D. A., Biggs, K. B. and Van Duyne, R. P., Surface-enhanced Raman spectroscopy of half-mustard agent, Analyst, 2006, 131, p. 568-572.

139. Sylvia, J. M., Janni, J. A., Klein, J. D. and Spencer, K. M., Surface-Enhanced Raman Detection of 2,4-Dinitrotoluene Impurity Vapor as a Marker To Locate Landmines, Anal Chem, 2000, 72, p. 5834-5840. 
140. Ko, H., Chang, S. and Tsukruk, V. V., Porous Substrates for Label-Free Molecular Level Detection of Nonresonant Organic Molecules, ACS Nano, 2008, 3, p. 181-188.

141. Xu, J. Y., Wang, J., Kong, L. T., Zheng, G. C., Guo, Z. and Liu, J. H., SERS detection of explosive agent by macrocyclic compound functionalized triangular gold nanoprisms, Journal of Raman Spectroscopy, 2011, 42, p. 1728-1735.

142. Sharma, B., Frontiera, R. R., Henry, A.-I., Ringe, E. and Van Duyne, R. P., SERS: Materials, applications, and the future, Materials Today, 2012, 15, p. 16-25. 


\section{Chapter 2-Horizontally Aligned One-Dimensional ZnO Nanonecklaces by CVD for SERS}

\section{$2.1 \quad$ Introduction}

$\mathrm{ZnO}$ is one of the most popular research topics in nanoscience and nanotechnology over the past several decades. A wide variety of $\mathrm{ZnO}$ nanostructures, such as nanowires, nanocombs, nanorings, nanowalls, nanocages, nanobows, nanobelts, nanodisk, nanopropeller, nanotubes, nanotetropods, nanocantilever, and nanohelixes, have been explored for diverse applications [1-13]. Among all these structures, one-dimensional ZnO nanostructures, such as nanowires and nanotubes, are of great importance because they are projected to be the fundamental components for many nanodevices $[7,8]$. Recently, there have been an increasing number of literatures on epitaxial growth of $\mathrm{ZnO}$ nanowires vertically or with certain angles on a-plane and c-plane sapphires using different methods. Particularly, more efforts have been made to fabricate horizontally aligned one-dimensional nanostructures 
since such nanostructures are compatible with conventional topdown microfabrication process and can be further processed for device assembly. These one dimensional nanostructures include but are not limited to Ge [14], GeSn [15], PtSi [16], silicides [17], In [18], $\mathrm{Ga}[18], \mathrm{CaF}_{2}[19], \mathrm{Bi}$ [20] quantum wires with diameters of only a few nanometers, $\mathrm{ZnO}[21], \mathrm{VO}_{2}[22], \mathrm{GaN}[23]$, and $\mathrm{In}_{2} \mathrm{O}_{3}$ [24] nanowires with diameters of tens of nanometers, and single walled carbon nanotubes [25],

The growth of one-dimensional nanowires differs very much from that of two-dimensional thin films because of the different growth mechanisms. The preferential growth direction of nanowires could be influenced by many factors, such as relatively high surface free energy of the growth surface, reduction of strain energy, catalysts-assisted growth process, relatively high anisotropic growth rate, etc. Tersoff et al. simulated the shape transition of $\mathrm{Ag}$ nanoislands to $\mathrm{Ag}$ nanowires on $\mathrm{Si}$ and found strain energy was relaxed by elongating the $\mathrm{Ag}$ island to an asymmetrical quantum wire with the aspect ratio as large as 1:50 [26]. Chen et al. summarized the growth behaviors of silicides quantum wires on $\mathrm{Si}$ wafer and found that asymmetrical one dimension growth phenomena happened when small lattice mismatch $(<2 \%)$ between deposited material and substrate existed along one direction while larger lattice mismatch existed along the perpendicular direction 
[17]. Based upon these work, anisotropic epitaxial relationship is speculated as one of the reasons for crystallographic alignment, which means a crystal wafer that has anisotropic epitaxial relationships with $\mathrm{ZnO}$ could be used as the substrate. Here, sapphire was selected as the growth substrate for following reasons. First, the lattice relationships between $\mathrm{ZnO}$ and all types of sapphire substrates ( $a, c, r$, and m-plane) satisfy anisotropic exitaxial requirement. As it is presented in Table 2.1, on a-plane, r-plane and m-plane sapphires, small lattice mismatches are present along one direction while large lattice mismatch along the perpendicular direction. Second, ZnO nanowires have been fabricated on a-plane and c-plane sapphires and $\mathrm{ZnO}$ thin films have been fabricated on a-plane, c-plane, and r-plane sapphires, which will provide us rich background information for experimental design and result analysis. Last, sapphire substrates have been demonstrated in horizontally growing one-dimensional carbon nanotubes [25, 27-29], and $\mathrm{VO}_{2}$ nanowires [22] in addition to $\mathrm{ZnO}$ nanowires [21,30]. Based upon above reasoning, we believe that horizontal $\mathrm{ZnO}$ nanostructure is able to be grown on sapphire substrates.

Table 2.1. Crystallographic relationships between $\mathrm{ZnO}$ and sapphire substrates.

\begin{tabular}{|c|c|c|}
\hline ZnO Plane II Sapphire Plane & $\begin{array}{l}\text { ZnO Direction II Sapphire } \\
\text { Direction (in plane) }\end{array}$ & $\begin{array}{l}\text { Lattice Mismatch } \\
\text { at } 25^{\circ} \mathrm{C}\end{array}$ \\
\hline \multirow{2}{*}{$\begin{array}{l}\text { ZnO (0001) II a-plane } \\
\text { sapphire (11-20) }\end{array}$} & $\begin{array}{c}\text { ZnO [11-20] || sapphire } \\
{[0001]}\end{array}$ & $0.08 \%$ \\
\hline & ZnO [-1100] || sapphire [- & $2.3 \%$ \\
\hline
\end{tabular}




\begin{tabular}{|c|c|c|}
\hline & 1100] & \\
\hline \multirow{2}{*}{$\begin{array}{l}\text { Zno (0001) || c-plane } \\
\text { sapphire (0001) }\end{array}$} & 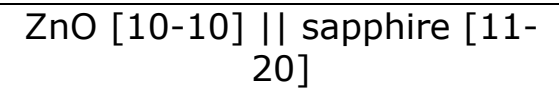 & $18.3 \%$ \\
\hline & $\begin{array}{c}\text { ZnO }[-12-10] \text { I| sapphire [- } \\
1100]\end{array}$ & $18.3 \%$ \\
\hline \multirow{2}{*}{$\begin{array}{c}\text { Zno }(11-20) \text { II r-plane } \\
\text { sapphire }(01-12)\end{array}$} & $\begin{array}{c}\text { ZnO [0001] II sapphire [10- } \\
11]\end{array}$ & $1.53 \%$ \\
\hline & $\begin{array}{l}\text { ZnO [10-10] || sapphire [2-1- } \\
10]\end{array}$ & $18.3 \%$ \\
\hline \multirow{2}{*}{$\begin{array}{c}\mathrm{ZnO}(01-10) \text { || m-plane } \\
\text { sapphire }(01-10)\end{array}$} & $\begin{array}{c}\mathrm{ZnO}[0001] \text { || sapphire [2-1- } \\
10]\end{array}$ & $9.1 \%$ \\
\hline & $\begin{array}{c}\text { ZnO [-2110] II sapphire } \\
{[0001]}\end{array}$ & $0.08 \%$ \\
\hline
\end{tabular}

Nanonecklaces (NNs), also known as nanochains or aligned nanoparticles, are usually in straight or curved lines with spherical or faceted nanoparticles, and have been made using metals [31-33], oxides [34], and other materials [35]. One of the most common methods to synthesize NN structures is hydrothermal self-assembly, which generally cannot provide a good control of the alignment and growth direction of NN arrays. In this chapter, chemical vapor deposition (CVD) is used to synthesize well aligned NN structures, which could be readily integrated for further processing with conventional microfabrication for device applications. Our ZnO NN structure fabricated using CVD method is the first ever discovered horizontally aligned faceted NN structure [36]. By controlling the density of the NN arrays, it is possible to control the distribution of "hot spots" on SERS substrates, hence providing reproducible SERS 
signals. Additionally, it is known that faceted structure yields much stronger Raman scattering than traditional spherical one, due to its sharper curvature resulting in higher electromagnetic effects [37]. Faceted structure has another benefit over spherical one in that it has higher free energies for the interactions with molecules, resulting in higher chemical effects [38].

As the first group that achieved a novel faceted horizontally aligned NN structure, we here explore the possibility of using these novel ZnO NN substrates for SERS applications. In this study, Au coatings with different thicknesses were sputtered onto the surface of these faceted ZnO NNs to activate SERS effects and the SERS performance was evaluated by detecting melamine and other chemicals at low concentration.

\subsection{Experimental Section}

\subsubsection{Fabrication of ZnO nanonecklaces}

In this work, a hot wall chemical vapor deposition (CVD) reactor was setup as shown in Fig. 2.1 to grow $\mathrm{ZnO}$ nanonecklaces (NNs) on r-plane sapphire substrates. Firstly, r-plane sapphire substrates were cleaned ultrasonically with isopropanol and deionized (DI) water, and then dried by nitrogen gas flow. Au nanoparticles in diameter of $5 \mathrm{~nm}$ were used as catalyst for $\mathrm{ZnO}$ growth. The Au nanoparticles were synthesized with tannic acid, 


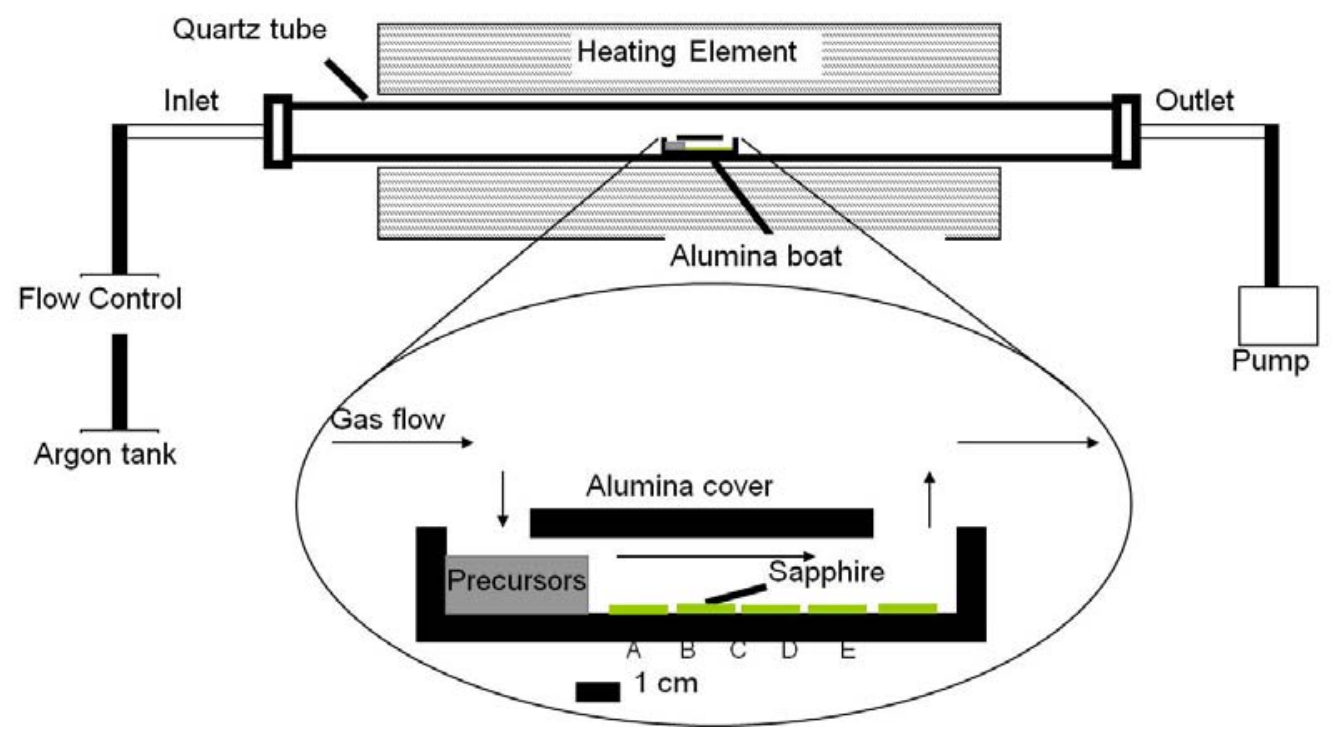

Figure 2.1. The schematic CVD reactor setup for $\mathrm{ZnO}$ NNs growth.

trisodium citrate, potassium carbonate and hydrogen tetrachloroaurate by following a reported method [39]. The Au nanopaticles suspension was then spin-coated (Special Coating System, P6700) twice on $3 \mathrm{~mm} \times 3 \mathrm{~mm}$ r-plane sapphire substrates at $6000 \mathrm{rpm}$. Secondly, a mixture of $\mathrm{ZnO}$ (99.99 \%) /graphite $(99.99 \%)$ with mass ratio of $2.5 \mathrm{~g}$ to $0.36 \mathrm{~g}$ was put in a $12 \mathrm{~cm}(\mathrm{~L})$ $\times 2 \mathrm{~cm}(\mathrm{~W}) \times 1 \mathrm{~cm}(\mathrm{H})$ alumina boat. The sapphire substrates were loaded and positioned down stream the carrier gas within the alumina boat as shown in the magnified region in Fig. 2.1. The samples at position A, B, C, D, and E, were approximately $3.1 \mathrm{~cm}$, $4.2 \mathrm{~cm}, 5.3 \mathrm{~cm}, 6.4 \mathrm{~cm}$, and $7.5 \mathrm{~cm}$ away from the center of the $\mathrm{ZnO} /$ graphite mixture. Then the alumina boat was loaded at the center of the single zone quartz tube $(47 \mathrm{~mm}$ in inner diameter and $120 \mathrm{~cm}$ in length). 
A series of experiments were conducted to investigate the influence of experimental parameters on the evolution of $\mathrm{ZnO}$ NNs. Typically, the experiment was conducted at $900{ }^{\circ} \mathrm{C}$ for $2 \mathrm{~h}$ with 60 standard cubic centimeter per minute $(\mathrm{sccm})$ Ar flowing at atmosphere pressure. Furnace was heated at the rate of $15^{\circ} \mathrm{C}$ per minute and cooled down naturally. Temperatures, including $700{ }^{\circ} \mathrm{C}$, $790{ }^{\circ} \mathrm{C}, 850{ }^{\circ} \mathrm{C}$, and $1000{ }^{\circ} \mathrm{C}$, and growth time, including $25 \mathrm{~min}, 45$ min, and $6 \mathrm{~h}$, were also tried to study their influences on ZnO NNs. After the fabrication, ZnO NNs substrates were sputtered with Au coatings at thicknesses of 45 and $60 \mathrm{~nm}$ by an Emitech $\mathrm{K} 575 \mathrm{x}$ turbo sputter coater. The coating thickness was based on Au mass and measured with a built-in film thickness monitor.

\subsubsection{Characterization of ZnO nanonecklaces}

A field emission scanning electron microscope (FESEM, S4700, Hitachi) and an atomic force microscope (AFM, Nanoscope IIIa, Digital Instruments) were used to characterize the morphology and topography of ZnO NNs with and without Au deposition.

\subsubsection{SERS measurement}

A Renishaw RM1000 Raman spectrometer system (Gloucestershire, UK) equipped with a Leica DMLB microscope (Wetzlar, Germany) and a $785 \mathrm{~nm}$ near-infrared diode laser source (maximum at $300 \mathrm{~mW}$ ) was used in this study. Raman scattering 
signals were detected by a $578 \times 385$ pixels CCD array detector. The size of each pixel was $22 \mu \mathrm{m} \times 22 \mu \mathrm{m}$. Spectra of each sample were collected using a $50 \times$ objective with a detection range from 300 to $1800 \mathrm{~cm}^{-1}$. The measurement was conducted with a $10 \mathrm{~s}$ exposure time and $\sim 15 \mathrm{~mW}$ laser power. Spectral data were analyzed using the software GRAMS 32 spectral notebase (Galactic Industries, Waltham, MA, USA).

A serial concentration of melamine (Sigma-Aldrich) in 50\% ethanol solution was used as the analyte to evaluate Au coated $\mathrm{ZnO}$ NNs substrate. Melamine is a food contaminant that was implicated in the pet food recalls in the North America in 2007 and recent infant formula contamination in China and the United States. In Melamine SERS spectra, the most intense peak around $683 \mathrm{~cm}^{-1}$ is assigned to the ring breathing mode II and involves in-plane deformation of the triazine ring; the second highest peak around $993 \mathrm{~cm}^{-1}$ arises from the ring breathing mode I of the triazine ring. In this study, the highest melamine peak $\left(\sim 683 \mathrm{~cm}^{-1}\right)$ was selected to correlate the melamine concentration with the peak intensity.

\subsection{Results and discussion}

\subsubsection{Morphology of ZnO Nanostructures}

Figure 2.2 depicts the typical FESEM images of the $\mathrm{ZnO}$ nanostructures grown at $900{ }^{\circ} \mathrm{C}$ for $2 \mathrm{~h}$ on r-plane (01-12) sapphire 
substrates. At the position $A$, which was very close to $(3 \mathrm{~cm})$ the $\mathrm{ZnO} /$ Graphite precursor, precursor concentration was relatively high and both nanonecklaces and nanoblades were observed. Facets were observed for all the ZnO nanonecklaces (NNs), indicative of crystalline structure. In addition, most of NNs show very similar facets and such similarity suggests that the NNs may grow with the same crystalline orientation. The same crystalline orientation of NNs can either result from a single crystalline growth and/or epitaxial growth, both of which were confirmed by transmission electron microscopy (TEM) [36]. The ZnO nanoblades are typically $200 \mathrm{~nm}$ $1 \mu \mathrm{m}$ in width and a few micrometers to a few tens micrometers in length and have multiple angles with substrates. The nanoblades were typically found grown out of the tip of nanonecklaces (Fig. 2.2(h)) and they also have different conformations, such as, nanorods. At positions $\mathrm{B}$ and $\mathrm{C}$, similar nanostructures as that of
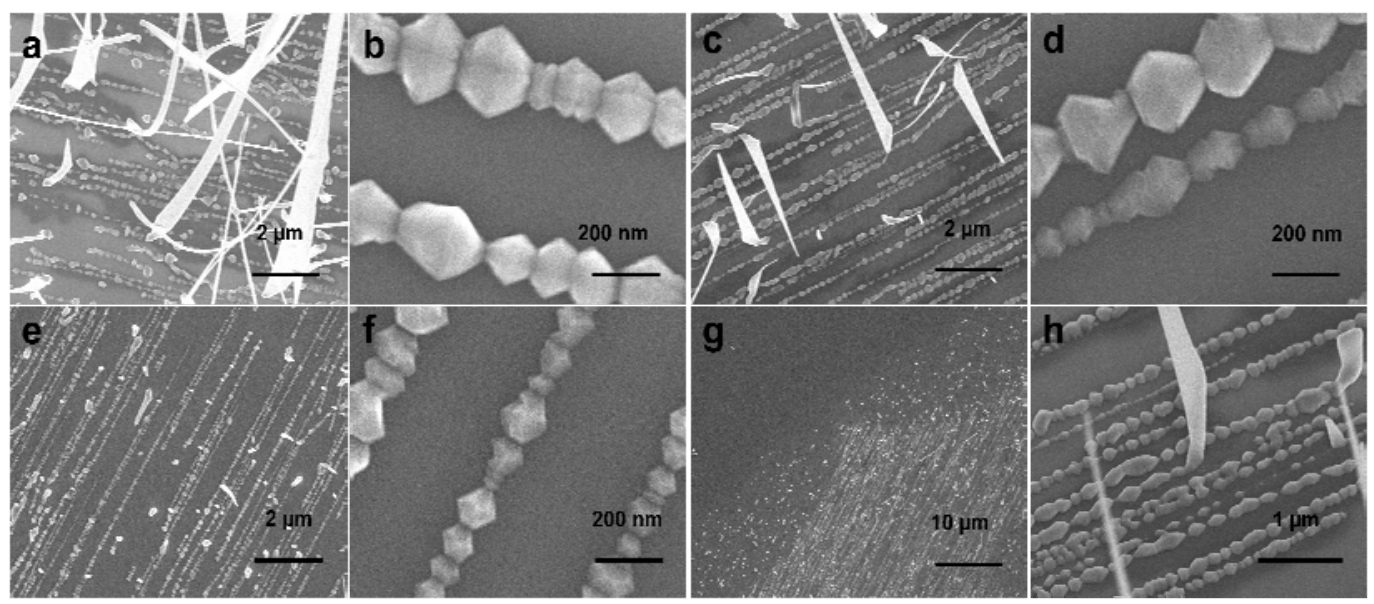

Figure 2.2. FESEM images of ZnO NNs fabricated at position $A((a)$ and (b)), $C((c)$ and $(d))$ and $E((e)$ and $(f)) ;(g)$ the boundary between Au nanoparticles uncoated and coated regions of sapphire substrate, leading to ZnO NNs free and present regions, respectively; (h) ZnO nanoblades. 
position A were observed, but ZnO NNs were thinner and shorter. And there were no discernible structural difference observed with FESEM between position $B$ and $C$. The nanoblades were no longer observed at positions $D$ and $E$ and these two positions also presented very similar structures. As shown in all the SEM images of Fig. 2.2, all the ZnO NNs align in one direction of r-plane sapphire substrates. Experiments also show that Au NPs are needed for the formation of $\mathrm{ZnO}$ nanostructures (Fig. 2.2(g)). In the areas that no AuNPs were deposited, no ZnO nanostructure was observed.

Figure 2.3(a) summarizes the width and length of $\mathrm{ZnO} N \mathrm{~N}$ at different positions. As a general trend, $\mathrm{ZnO} \mathrm{NNs}$ are getting thinner and shorter when the sapphire substrates are further from the graphite/ZnO mixture precursor source. Such differences in width and length at different positions could be attributed to the decreasing precursor concentration along the Ar carrier gas flow direction. The influence of the growth time and growth temperature on the ZnO NN growth was also studied. Figure $\mathbf{2 . 3 ( b )}$ shows that both the length and width of $\mathrm{ZnO} \mathrm{NN}$ increase with growth time. The length increase was due to the catalyst assisted growth. For the $2 \mathrm{~h}$ growth, the standard deviation is very large and we speculate that this is due to the formation of new $\mathrm{ZnO} \mathrm{NNs}$ during the twohour growth period. As a result, some $\mathrm{ZnO}$ NNs have much shorter actual growth time than $2 \mathrm{~h}$ and their lengths are expected to be 
much shorter. The width increase was also observed, indicating the direct deposition of $\mathrm{ZnO}$ on $\mathrm{ZnO}$ NNs after its initial growth. There is also a linear relationship between the $\mathrm{ZnO} \mathrm{NN}$ width and the growth time. The trend line intercepts with width axis at $20 \mathrm{~nm}$ and with a slope of $100 \mathrm{~nm} / \mathrm{h}$. The $20 \mathrm{~nm}$ could be explained as the initial $\mathrm{ZnO}$ NN width formed directly from catalyst assisted growth through the vapor-liquid-solid (VLS) growth mechanism before any direct ZnO deposition from vapor phase. It is worth noting that $20 \mathrm{~nm}$ is bigger than the Au NP catalyst size of $5 \mathrm{~nm}$, but they are at the same scale. In addition, the growth temperature also significantly influenced the morphology of ZnO growth on r-plane sapphire. ZnO NNs were only observed in experiments conducted at $850{ }^{\circ} \mathrm{C}$ and $900{ }^{\circ} \mathrm{C}$. Porous nanorods were synthesized rather than $\mathrm{ZnO} N \mathrm{Ns}$ at $700{ }^{\circ} \mathrm{C}$, whereas ZnO thin films were observed at $1000{ }^{\circ} \mathrm{C}$.

At this stage, experimental conditions for optimal $\mathrm{ZnO}$ NNs
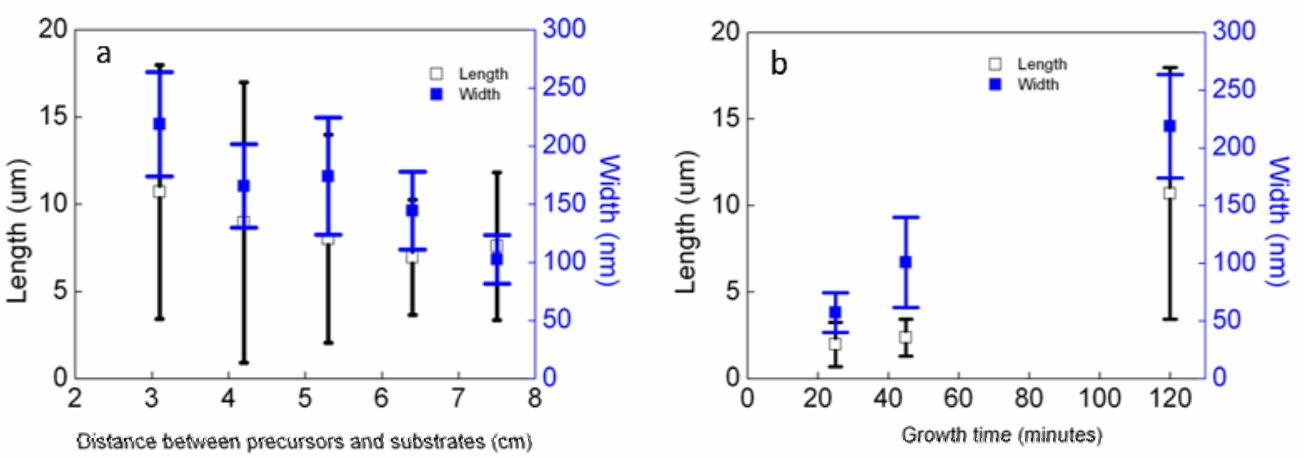

Figure 2.3. (a) The length and width of ZnO NNs vs the distance between precursors and substrates; (b) the length and width of ZnO NNs vs the growth time. 
(Fig. 2.2(c)-(d)) have been identified as: substrate position between $C$ and $D$, growth temperature of $900{ }^{\circ} \mathrm{C}$, and growth time of $2 \mathrm{~h}$. Following studies will be focused on samples prepared under such conditions. Please note that we set substrate position as 60 $\mathrm{mm}$ away from precursors.

An AFM image and sectional analysis of the ZnO NNs on rplane sapphire substrates was presented in Fig. 2.4. This result shows well connected NN structure and roughly patterned surface,

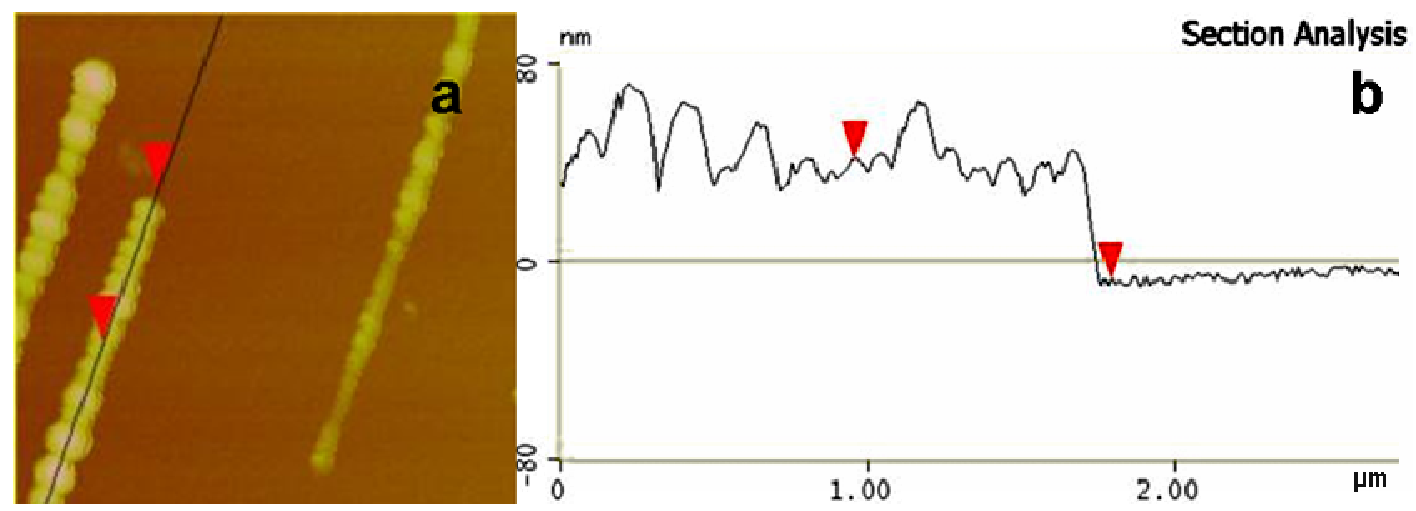

Figure 2.4. AFM image (a) and section analysis profile (b) of the ZnO NNs.

which is very likely to provide a large quantity of periodical SERS "hot spots" that are key to SERS.

\subsubsection{SERS measurement}

Figure 2.5 indicates that SERS signals acquired from the Au coated $\mathrm{ZnO} N N$ substrate with $45 \mathrm{~nm}$ Au coating were stronger than that from $60 \mathrm{~nm}$. Here, we propose two possible reasons to explain this difference. First, it is likely that thicker coating led to a 
smoother substrate surface with less sharp edge or fewer edges from the underneath faceted structures and consequently caused the decrease of the SERS signals. The second reason could be illustrated from the perspective of surface plasmon and its dependence on materials configuration. Basically, the Au coated ZnO NN could be considered as an incomplete core-shell structure (ZnO nanoparticle core not fully covered by $\mathrm{Au}$ coating shell). Regarding to core-shell structure, Prodan et al. [40] suggested that the plasmon resonance can be interpreted as an interaction between the essentially fixed-frequency plasmon response of a nanosphere and that of a nanocavity. And the plasmon resonance of nanoshell is highly tunable by adjusting the thickness of the Au coating. Therefore, the reason for weakened SERS sensitivity with thicker Au coating might be that the plasmon resonance was tuned

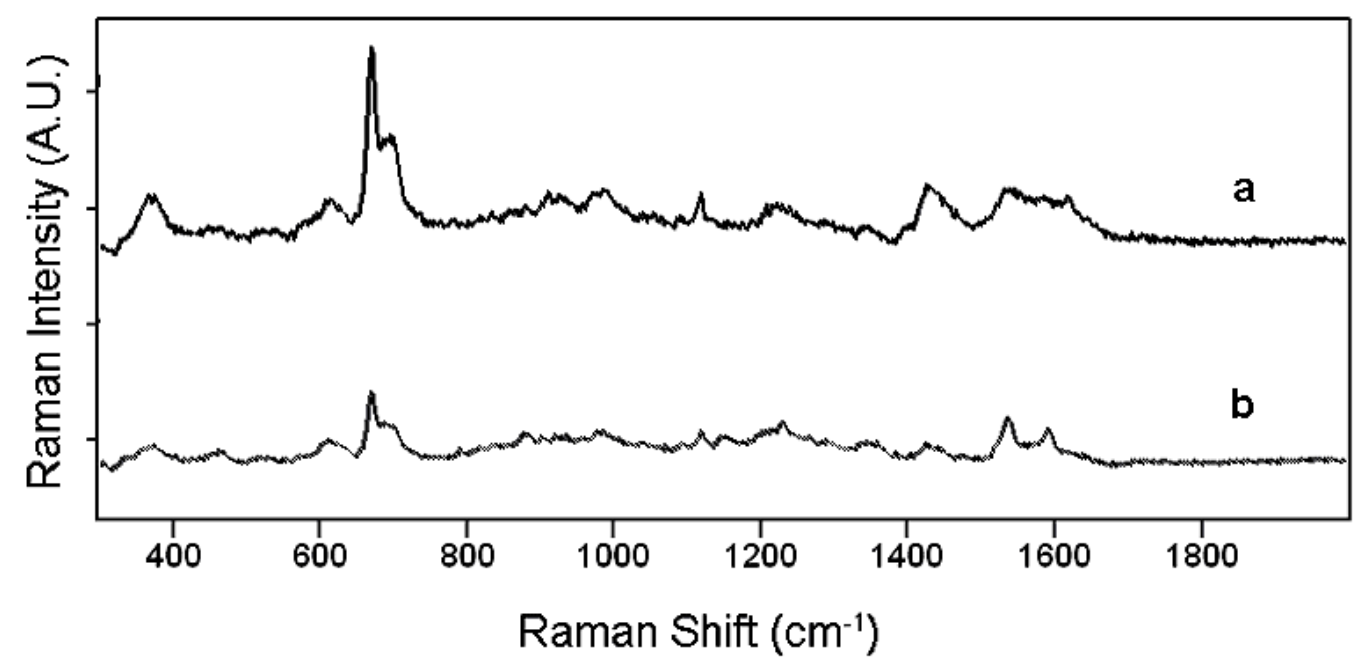

Figure 2.5. Average Raman spectra $(n=3)$ of $10^{-4} \mathrm{~mol} / \mathrm{L}$ melamine on a $\mathrm{ZnO}$ NN substrate with $45 \mathrm{~nm}$ (a) and $60 \mathrm{~nm}$ (b) Au coating. Raman measurements were taken under the same conditions. 
away from the $785 \mathrm{~nm}$ excitation frequency for a $60 \mathrm{~nm}$ Au coating. Based on the result of Fig. 2.5, we decided to use $45 \mathrm{~nm} \mathrm{Au}$ coating for the rest of the study.

In order to evaluate SERS analytical enhancement factor (AEF), an Au coated ZnO NN substrate and a plain Au film substrate were used for calculation. AEF is defined as follows,

$$
\operatorname{AEF}=\left(\mathrm{C}_{\mathrm{R}} / \mathrm{C}_{\mathrm{SERS}}\right) *\left(\mathrm{I}_{\mathrm{SERS}} / \mathrm{I}_{\mathrm{R}}\right)
$$

where $C_{S E R S}$ and $C_{R}$ are concentrations of analyte solutions for SERS and non-SERS measurements, respectively. $I_{S E R S}$ and $I_{R}$ are Raman intensities of the same peak for SERS and non-SERS measurements

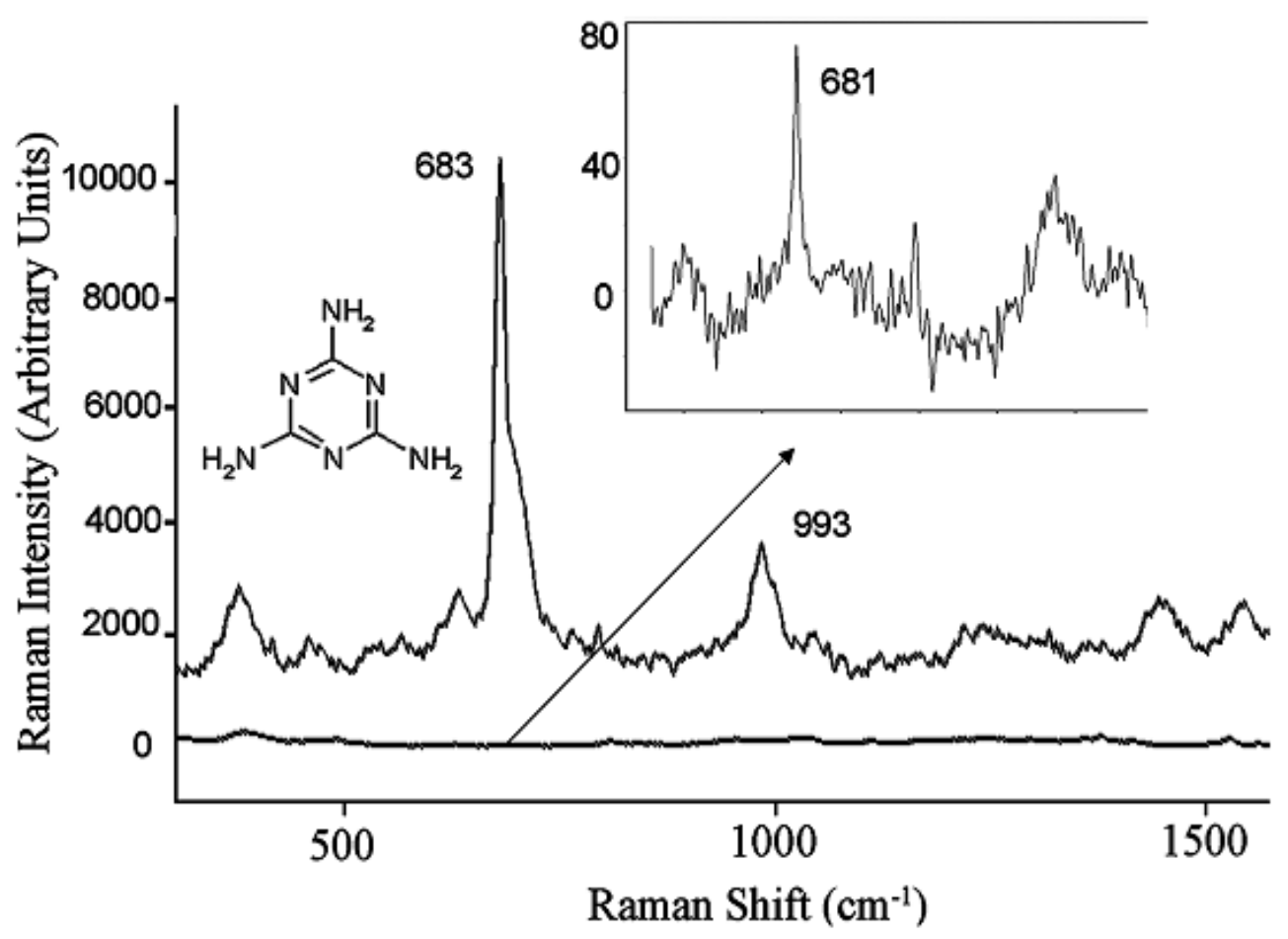

Figure 2.6. Average Raman spectra $(\mathrm{n}=3)$ of $10^{-4} \mathrm{~mol} / \mathrm{L}$ melamine on a $\mathrm{Au}$ coated $\mathrm{ZnO} N N$ substrate and $10^{-2} \mathrm{~mol} / \mathrm{L}$ melamine (inset) on a Au film substrate. Raman measurements were taken under the same conditions. 
performed under exactly same conditions. Figure $\mathbf{2 . 6}$ shows their SERS spectra of melamine at different concentrations. No or low interfering signals from the background of the substrate were observed. By comparing the peak intensity $\left(683 \mathrm{~cm}^{-1}\right)$ from the $\mathrm{Au}$ coated ZnO NN substrate with that from a plain Au film substrate, the AEF was determined to be higher than $10^{4}$, which is competitive with a commercial Au based SERS substrate.

Figure 2.7 shows the SERS spectra of different concentrations of melamine $\left(10^{-6}, 10^{-5}, 10^{-4}, 10^{-3}\right.$, and $\left.10^{-2} \mathrm{~mol} / \mathrm{L}\right)$ analyzed using Au coated ZnO NNs. Melamine characteristic peaks could be still observed when the concentration was down to $10^{-5}$ $\mathrm{mol} / \mathrm{L}$. Figure $\mathbf{2 . 8}$ shows the relationship between melamine concentrations and the intensity of Raman signals of the melamine peak at $683 \mathrm{~cm}^{-1}$. A linear regression $\left(R^{2}=0.9561\right)$ was established between log value of melamine concentration and Raman intensity of the peak at around $683 \mathrm{~cm}^{-1}$. The error bars in Fig. 2.8 indicate the standard deviation of Raman intensity of signals acquired from three randomly picked spots on the substrate. These results suggest that SERS method coupled with ZnO NNs has a great potential for quantitative analysis, though, further research is needed to improve the performance. Compared to the current expensive and time consuming analytical techniques, SERS could be an alternative 
method for qualitative and quantitative analysis with a large capacity.

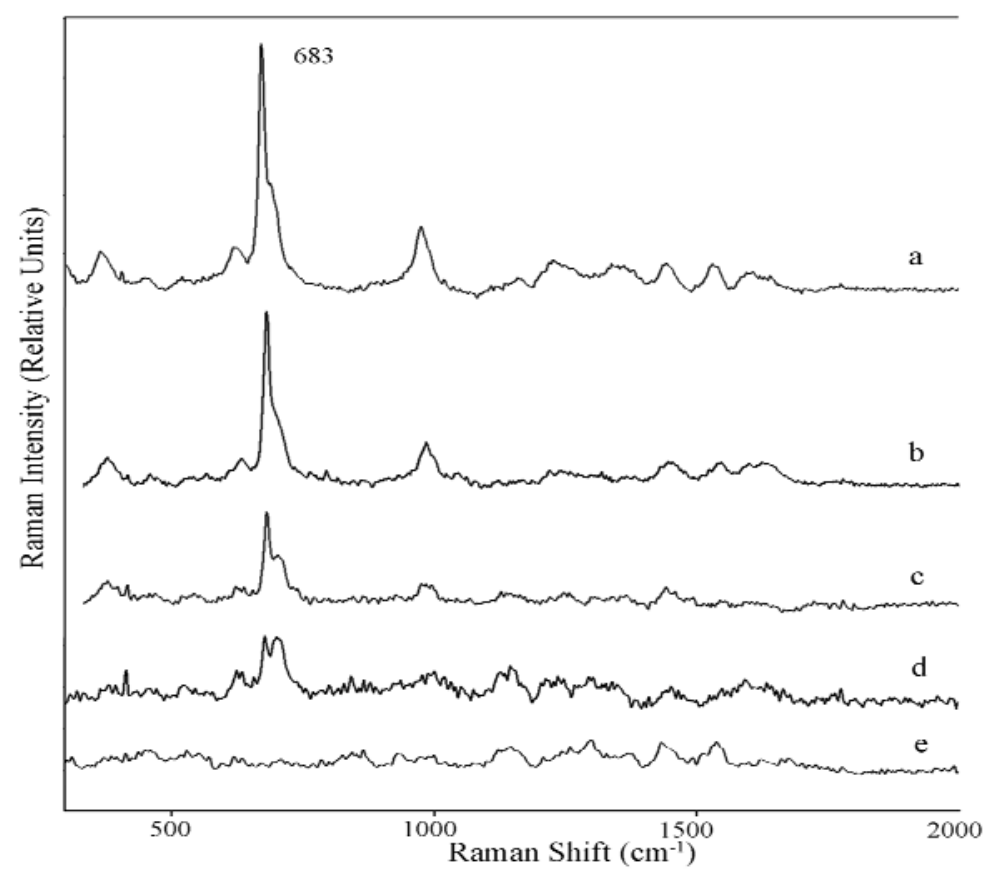

Figure 2.7. Average SERS spectra of a serial concentration of melamine (a: $10^{-2}, \mathrm{~b}: 10^{-3}, \mathrm{c}: 10^{-4}, \mathrm{~d}: 10^{-5}$, and e: $10^{-6} \mathrm{~mol} / \mathrm{L}$ ) deposited onto Au coated ZnO NNs. 


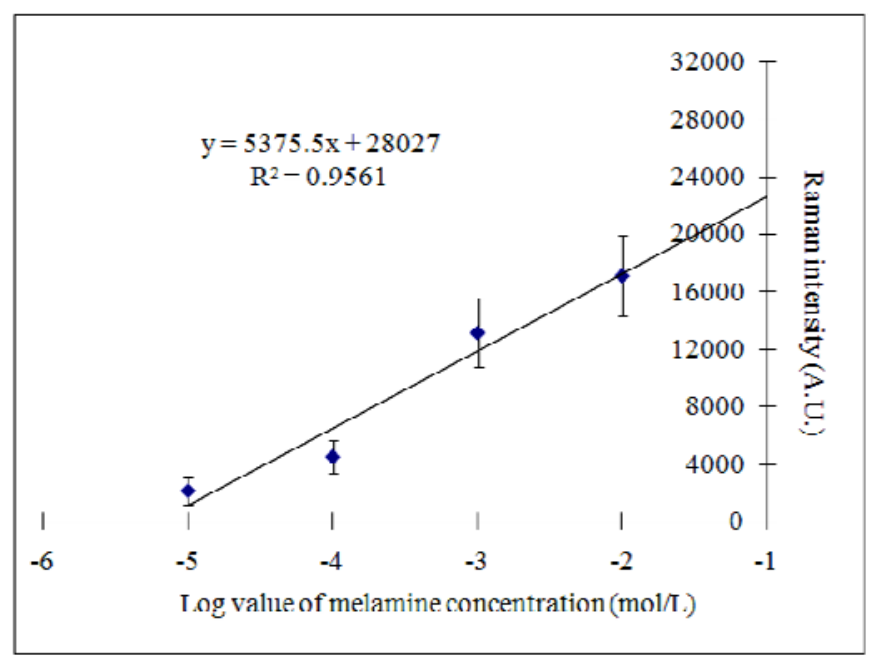

Figure 2.8. Relationship between log value of melamine concentration (mol/L) and Raman intensity of a melamine peak at $683 \mathrm{~cm}^{-1}$ (average of three spectra).

Two more chemical food contaminants, namely crystal violet (CV) and malachite green (MG), were chosen to test the SERS selectivity of these Au coated ZnO NN substrates. Both CV and MG

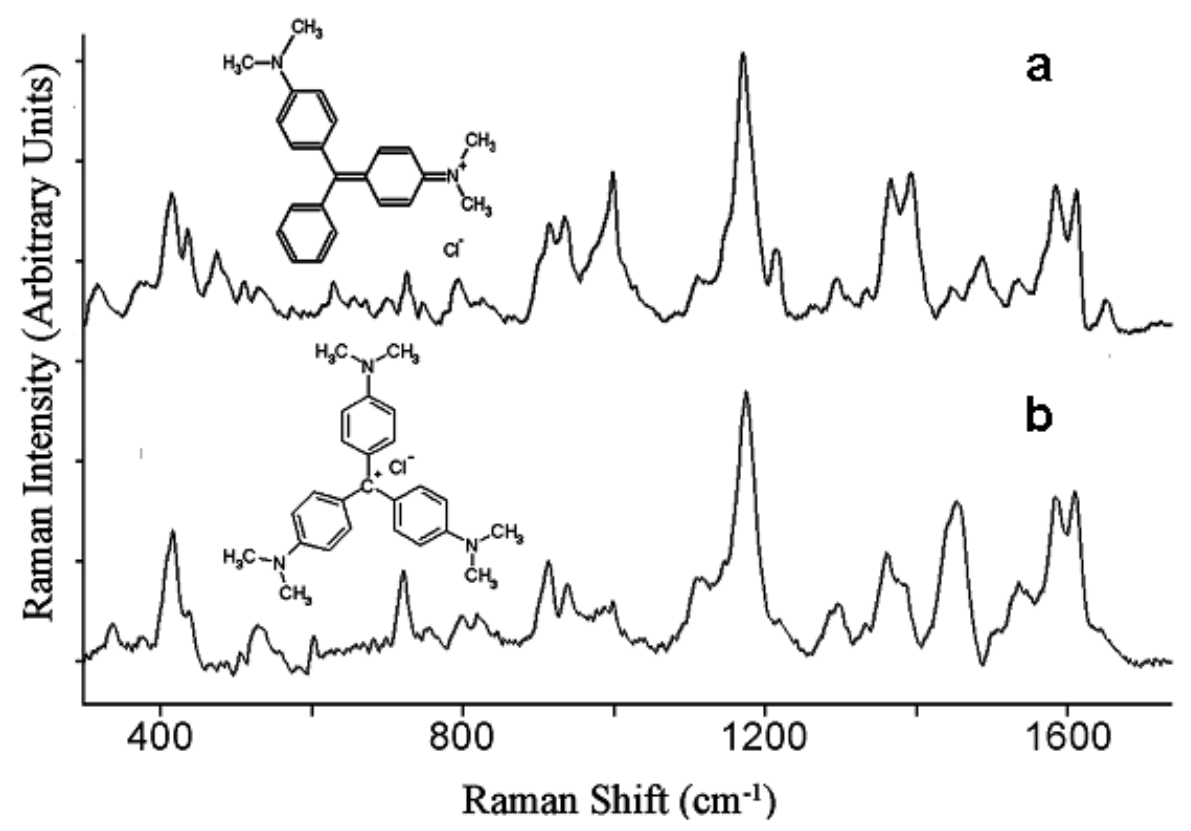

Figure 2.9. Average Raman spectra $(\mathrm{n}=3)$ of $10^{-4} \mathrm{~mol} / \mathrm{L}$ malachite green (a) and $10^{-4} \mathrm{~mol} / \mathrm{L}$ crystal violet (b) on an Au coated ZnO NN substrate. 
were implicated in 2007 seafood alert in the US. Zero tolerance policy has been set for the residues of CV and MG in fish by the US Food and Drug Administration (FDA) [41]. These two compounds have very similar chemical structures, both more complex than melamine, making themselves perfect candidates for evaluating the selectivity of SERS substrates. Distinctive Raman spectra of these two chemicals were shown in Fig. 2.9(a) and (b), respectively. Although appearing very similar, these two spectra present critical features to differentiate each other, in accordance with our previous study [42]. In short, the result in Fig. 2.9 demonstrates that $\mathrm{Au}$ coated $\mathrm{ZnO} \mathrm{NN}$ substrate is capable of identifying a variety of chemicals with good specificity.

As mentioned in section 2.3.1, when sapphire substrates were positioned at different positions $(50,40,30,20 \mathrm{~mm})$ away from the precursors, nanoblades (NBs) ZnO was obtained along with different ZnO NNs. Figure 2.10 shows nanoblades (NBs) structure with a certain angle grown upon the NNs. The NBs were typically found grown out of the tip of NNs. It was observed that more ZnO NBs and NNs were grown on the sapphire substrate as they were closer to precursor. Compared to NNs, NBs decreased more dramatically with increasing distance away from the precursor. When substrate was placed $60 \mathrm{~mm}$ away from the precursor, almost all nanostructures were NNs. It was discussed in our previous study 
that the Au NPs with diameter of $\sim 5 \mathrm{~nm}$ and lower density of such NPs are critical for the formation of horizontally aligned ZnO NN arrays while higher density or bigger size of Au NPs resulted in vertical ZnO NBs [36].

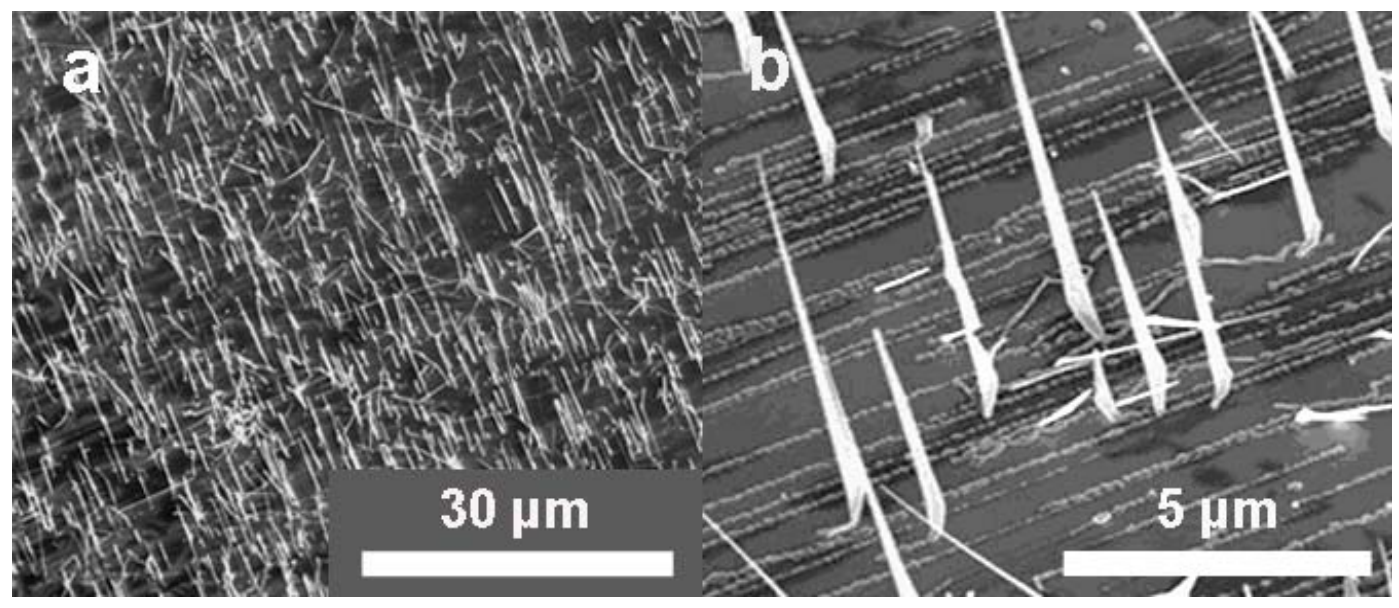

Figure 2.10. SEM images of $\mathrm{ZnO}$ nanoblades and nanonecklaces grown on sapphire substrate that was $20 \mathrm{~mm}$ away from the precursors.

SERS effects from five Au-coated $\mathrm{ZnO}$ NN substrates at different positions $(60,50,40,30,20 \mathrm{~mm})$ were evaluated using melamine $\left(10^{-4} \mathrm{~mol} / \mathrm{L}\right)$ as the analyte. As shown in Fig. 2.11, the substrate at $40 \mathrm{~mm}$ position yielded the highest SERS effects among all samples. We propose explanations to this result in two aspects. On one hand, it appears that the presence of NB was responsible for the lower SERS effects of near field samples (20 and $30 \mathrm{~mm}$ ). There are several factors leading to such claim. First, these long tilted NBs in micrometer may reduce the reflectivity of the substrate and partially block Raman scattering signals. Second, the NBs may impede the Au sputtering process and prevent Au from covering bottom $\mathrm{ZnO}$ and thus influence the SERS effects. On the 


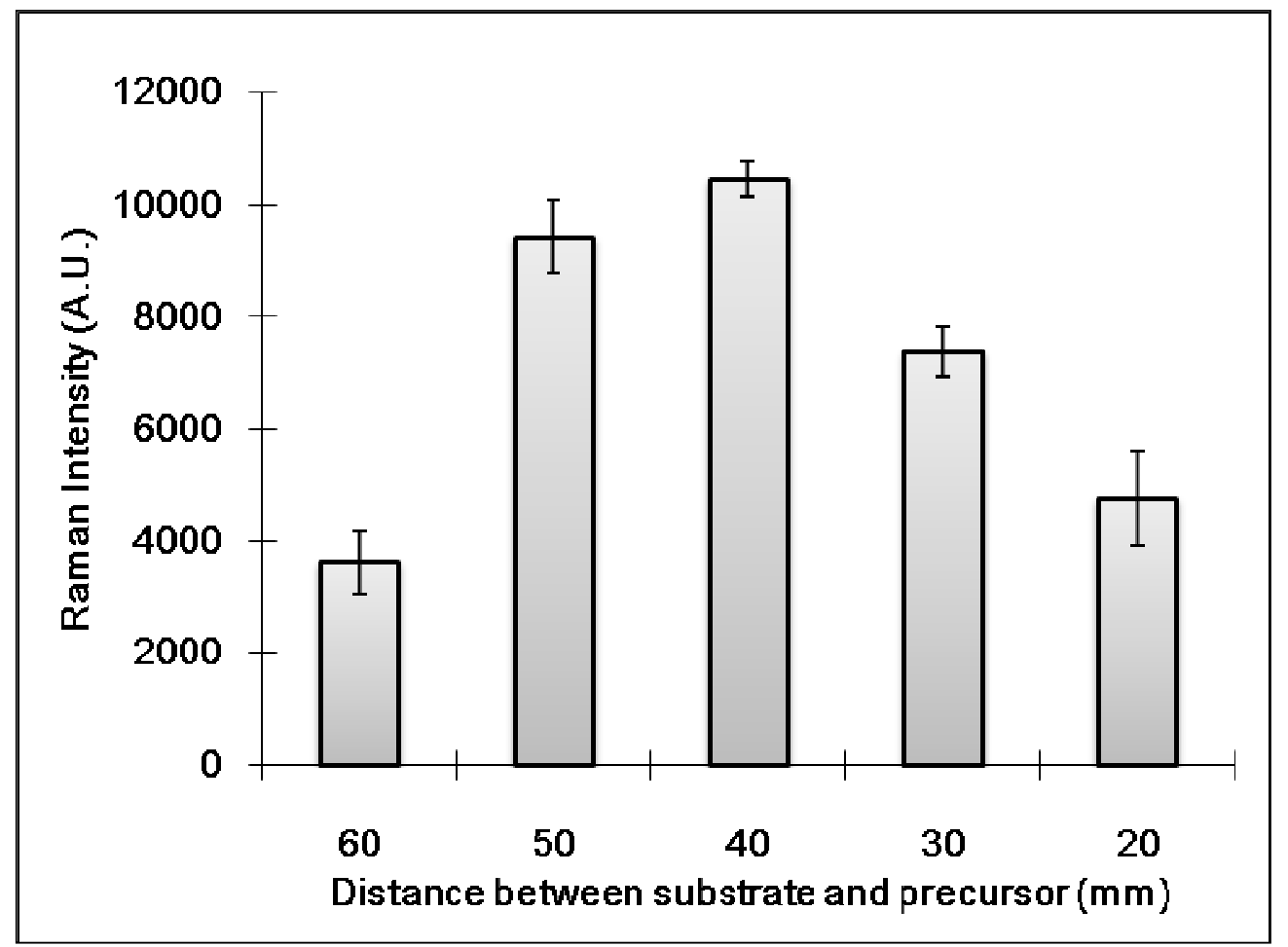

Figure 2.11. Raman intensity of melamine $\left(10^{-4} \mathrm{~mol} / \mathrm{L}\right)$ peak at $683 \mathrm{~cm}^{-1}$ acquired from five different $\mathrm{ZnO} N \mathrm{~N}$ substrates (average of three spectra). All substrates were coated with $\sim 45 \mathrm{~nm}$ Au coating.

other hand, the decrease of SERS effects of far field samples (50 and $60 \mathrm{~nm}$ ) was possibly due to the lower density of NNs. We would like to declare that the $\mathrm{ZnO} \mathrm{NN}$ arrays with different densities were intentionally designed in the present study to investigate the influence of ZnO NN structure on SERS effects. Uniform ZnO NN arrays with desired density could be achieved by precisely controlling the precursor concentration, such as using a shower head design in a commercial CVD system.

\subsection{Conclusion}


In summary, we investigated the potential SERS application of novel faceted $\mathrm{ZnO} \mathrm{NN}$ arrays that were horizontally-aligned on rplane sapphire substrates. Our results show that Au coated $\mathrm{ZnO} N \mathrm{~N}$ arrays can serve as a viable substrate for SERS applications and provide satisfactory enhancement when used to detect different analytes such as melamine and other food contaminates. It was found that $\mathrm{ZnO} \mathrm{NNs}$ contributed to the SERS effects while ZnO NBs, when grown with larger Au nanoparticles or higher density of Au nanoparticles as catalysts in the CVD process, reduced the SERS enhancement factor. We expect that $\mathrm{ZnO} \mathrm{NN}$ arrays at wafer scale could be fabricated with reproducible structures by precisely controlling the experimental conditions. Despite promising preliminary data, more work needs to be conducted to 1) further improve the uniformity and the density of ZnO NN arrays and 2) optimize the thickness of the Au coating. In addition, different coating techniques can be explored for further improvement of consistency of the ZnO NN based SERS substrates.

\section{$2.5 \quad$ Reference}

1. Wang, Z. L., Novel nanostructures of ZnO for nanoscale photonics, optoelectronics, piezoelectricity, and sensing, Applied Physics A: Materials Science \& Processing, 2007, 88, p. 7-15.

2. Lieber, C. M. and Wang, Z. L., Functional Nanowires, MRS Bulletin, 2007, 32, p. $99-108$. 
3. Ding, Y. and Wang, Z. L., Structure Analysis of Nanowires and Nanobelts by Transmission Electron Microscopy, The Journal of Physical Chemistry B, 2004, 108, p. 12280-12291.

4. Fu, Z., Wang, Z., Yang, B., Yang, Y., Yan, H. and Xia, L., Shape-control of nano-ZnO by changing the solvent, Materials Letters, 2007, 61, p. 48324835.

5. Gao, P. and Wang, Z. L., Self-Assembled Nanowire-Nanoribbon Junction Arrays of ZnO, The Journal of Physical Chemistry B, 2002, 106, p. 1265312658.

6. Gao, P. X. and Wang, Z. L., Nanopropeller arrays of zinc oxide, Applied Physics Letters, 2004, 84, p. 2883-2885.

7. Greene, L. E., Yuhas, B. D., Law, M., Zitoun, D. and Yang, P., SolutionGrown Zinc Oxide Nanowires, Inorganic Chemistry, 2006, 45, p. 75357543.

8. Wang, X., Song, J., Liu, J. and Wang, Z. L., Direct-Current Nanogenerator Driven by Ultrasonic Waves, Science, 2007, 316, p. 102-105.

9. Wang, X. D., Summers, C. J. and Wang, Z. L., Mesoporous Single-Crystal ZnO Nanowires Epitaxially Sheathed with Zn2SiO4, Advanced Materials, 2004, 16, p. 1215-1218.

10. Wang, Z. L., Kong, X. Y. and Zuo, J. M., Induced Growth of Asymmetric Nanocantilever Arrays on Polar Surfaces, Physical Review Letters, 2003, 91, p. 185502.

11. Yang, P., Yan, H., Mao, S., Russo, R., Johnson, J., Saykally, R., Morris, N., Pham, J., He, R. and Choi, H. J., Controlled Growth of ZnO Nanowires and Their Optical Properties, Advanced Functional Materials, 2002, 12, p. 323331.

12. Kong, X. Y., Ding, Y. and Wang, Z. L., Metal-Semiconductor Zn-ZnO Core-Shell Nanobelts and Nanotubes, The Journal of Physical Chemistry B, 2003, 108, p. 570-574.

13. Wang, Z. L., Nanostructures of zinc oxide, Materials Today, 2004, 7, p. 26-33.

14. Sunamura, H., Usami, N., Shiraki, Y. and Fukatsu, S., Observation of lateral confinement effect in Ge quantum wires self-aligned at step edges on Si(100), Applied Physics Letters, 1996, 68, p. 1847-1849. 
15. Deng, X., Yang, B. K., Hackney, S. A., Krishnamurthy, M. and Williams, D. R. M., Formation of Self-Assembled Quantum Wires during Epitaxial Growth of Strained GeSn Alloys on Ge(100): Trench Excavation by Migrating Sn Islands, Physical Review Letters, 1998, 80, p. 1022-1025.

16. Kavanagh, K. L., Reuter, M. C. and Tromp, R. M., High-temperature epitaxy of PtSiSi(l 0 1), Journal of Crystal Growth, 1997, 173, p. 393-401.

17. Chen, Y., Ohlberg, D. A. A. and Williams, R. S., Nanowires of four epitaxial hexagonal silicides grown on Si(001), Journal of Applied Physics, 2002, 91, p. 3213-3218.

18. Evans, M. M. R. and Nogami, J., Indium and gallium on Si(001): A closer look at the parallel dimer structure, Physical Review B, 1999, 59, p. 76447648.

19. Viernow, J., Petrovykh, D. Y., Men, F. K., Kirakosian, A., Lin, J. L. and Himpsel, F. J., Linear arrays of CaF[sub 2] nanostructures on Si, Applied Physics Letters, 1999, 74, p. 2125-2127.

20. Miki, K., Bowler, D. R., Owen, J. H. G., Briggs, G. A. D. and Sakamoto, K., Atomically perfect bismuth lines on Si(001), Physical Review B, 1999, 59, p. $14868-14871$.

21. Nikoobakht, B., Michaels, C. A., Stranick, S. J. and Vaudin, M. D., Horizontal growth and in situ assembly of oriented zinc oxide nanowires, Applied Physics Letters, 2004, 85, p. 3244-3246.

22. Sohn, J. I., Joo, H. J., Porter, A. E., Choi, C.-J., Kim, K., Kang, D. J. and Welland, M. E., Direct Observation of the Structural Component of the Metal-Insulator Phase Transition and Growth Habits of Epitaxially Grown VO2 Nanowires, Nano Letters, 2007, 7, p. 1570-1574.

23. Jian, J. K., Wang, C., Lei, M., Zhang, Z. H., Wang, T. M. and Chen, X. L., Quasi-horizontal GaN nanowire array network grown by sublimation sandwich technique, Applied Surface Science, 2008, 254, p. 6637-6641.

24. Hsin, C. L., He, J. H., Lee, C. Y., Wu, W. W., Yeh, P. H., Chen, L. J. and Wang, Z. L., Lateral Self-Aligned p-Type In2O3 Nanowire Arrays Epitaxially Grown on Si Substrates, Nano Letters, 2007, 7, p. 1799-1803.

25. Ago, H., Nakamura, K., Ikeda, K.-i., Uehara, N., Ishigami, N. and Tsuji, M., Aligned growth of isolated single-walled carbon nanotubes programmed by atomic arrangement of substrate surface, Chemical Physics Letters, 2005, 408, p. 433-438. 
26. Tersoff, J. and Tromp, R. M., Shape transition in growth of strained islands: Spontaneous formation of quantum wires, Physical Review Letters, 1993, 70, p. 2782-2785.

27. Ago, H., Uehara, N., Ikeda, K.-i., Ohdo, R., Nakamura, K. and Tsuji, M., Synthesis of horizontally-aligned single-walled carbon nanotubes with controllable density on sapphire surface and polarized Raman spectroscopy, Chemical Physics Letters, 2006, 421, p. 399-403.

28. Han, S., Liu, X. and Zhou, C., Template-Free Directional Growth of SingleWalled Carbon Nanotubes on a- and r-Plane Sapphire, Journal of the American Chemical Society, 2005, 127, p. 5294-5295.

29. Yu, Q., Qin, G., Li, H., Xia, Z., Nian, Y. and Pei, S.-S., Mechanism of Horizontally Aligned Growth of Single-Wall Carbon Nanotubes on R-Plane Sapphire, The Journal of Physical Chemistry B, 2006, 110, p. 2267622680.

30. Nikoobakht, B., Toward Industrial-Scale Fabrication of Nanowire-Based Devices, Chemistry of Materials, 2007, 19, p. 5279-5284.

31. Yang, Y., Shi, J., Tanaka, T. and Nogami, M., Self-Assembled Silver Nanochains for Surface-Enhanced Raman Scattering, Langmuir, 2007, 23, p. $12042-12047$.

32. Qin, Y., Lee, S.-M., Pan, A., Gosele, U. and Knez, M., Rayleigh-InstabilityInduced Metal Nanoparticle Chains Encapsulated in Nanotubes Produced by Atomic Layer Deposition, Nano Letters, 2007, 8, p. 114-118.

33. Zhan, Y.-J. and Yu, S.-H., Necklace-Like Cu@Cross-Linked Poly(vinyl alcohol) Core-Shell Microcables by Hydrothermal Process, Journal of the American Chemical Society, 2008, 130, p. 5650-5651.

34. Li, Z., Xu, F., Sun, X. and Zhang, W., Oriented Attachment in Vapor: Formation of ZnO Three-Dimensional Structures by Intergrowth of ZnO Microcrystals, Crystal Growth \& Design, 2008, 8, p. 805-807.

35. Maheshwari, V. and Saraf, R. F., Mineralization of Monodispersed CdS Nanoparticles on Polyelectrolyte Superstructure Forming an Electroluminescent "Necklace-of-Beads", Langmuir, 2006, 22, p. 86238626.

36. Shi, J., Sun, X., Zhang, J., Lian, J., Yu, Q., Lin, M. and Li, H., Epitaxial Growth of Horizontally Aligned Zinc Oxide Nanonecklace Arrays on r-Plane Sapphire, The Journal of Physical Chemistry C, 2009, 113, p. 2084520854. 
37. Hao, E. and Schatz, G. C., Electromagnetic fields around silver nanoparticles and dimers, The Journal of Chemical Physics, 2004, 120, p. 357-366.

38. Zhang, J., Li, X., Sun, X. and Li, Y., Surface Enhanced Raman Scattering Effects of Silver Colloids with Different Shapes, The Journal of Physical Chemistry B, 2005, 109, p. 12544-12548.

39. Vispute, R. D., Talyansky, V., Choopun, S., Sharma, R. P., Venkatesan, T., He, M., Tang, X., Halpern, J. B., Spencer, M. G., Li, Y. X., Salamanca-Riba, L. G., Iliadis, A. A. and Jones, K. A., Heteroepitaxy of ZnO on GaN and its implications for fabrication of hybrid optoelectronic devices, Applied Physics Letters, 1998, 73, p. 348-350.

40. Prodan, E., Radloff, C., Halas, N. J. and Nordlander, P., A Hybridization Model for the Plasmon Response of Complex Nanostructures, Science, 2003, 302, p. 419-422.

41. FDA. 2007; Available from: http://www.foodsafety.gov/ dms/fs-toc.html

42. He, L., Kim, N.-J., Li, H., Hu, Z. and Lin, M., Use of a Fractal-like Gold Nanostructure in Surface-Enhanced Raman Spectroscopy for Detection of Selected Food Contaminants, Journal of Agricultural and Food Chemistry, 2008, 56, p. 9843-9847. 


\section{Chapter 3-Au Nanoisland Arrays by Repeated Deposition and Post-Deposition Annealing for}

SERS

\subsection{Introduction}

Metal island films have long been employed in a wide variety of research areas as this class of nanometer scaled material is readily accessible with low cost laboratory equipments. It is well known that, at temperatures well below the melting point, surface melting migration causes metal films of 0.5 to $10 \mathrm{~nm}$ thickness to form a stochastic array of particulates in the form of spheroid on such substrate as $\mathrm{Si}$, quartz, or glass $[1,2]$. Over the past two decades, metal island films have attracted particular interests as they do not behave, optical wise, similarly to the corresponding bulk materials [3]. In fact, the discontinuous island films interact with the incoming light over an interesting phenomenon-surface plasmon [4]. Due to this unique property, metal island films have become important elements of present-day optics, optoelectronics and chemical and biological 
sensors [5-9]. Recently, an increasing number of reports have demonstrated that $\mathrm{Au}$ and $\mathrm{Ag}$ island films are promising candidates for SERS $[10,11]$

Herein, we report a simple SERS substrates fabrication technique on the basis of Au island film, as schematically illustrated in Fig. 3.1, which only involves controllable and scalable sputtering and heattreatment processes. The technique, primarily based on solid-state dewetting, was developed to create quasi-periodic Au nanoisland arrays without involving other complicated procedures, such as lithography [12-14] or template $[15,16]$ methods. While Au and Ag island films by physical vapor deposition and post-deposition annealing have been reported elsewhere [17-24], our work distinguished from those studies with a particular design of repeated processes of deposition and annealing, leading to nanoisland arrays with wellcontrolled size for SERS applications. With such design, we were able to achieve uniform nanoisland arrays with proper gaps that demonstrated promising SERS performance. Compared with the periodic nanostructures created by lithography and template methods, our processes are simpler, more reliable, and more cost-effective. In addition, our research also provides a simple way of investigating the influence of size and spacing of nanoparticles on SERS performance. 


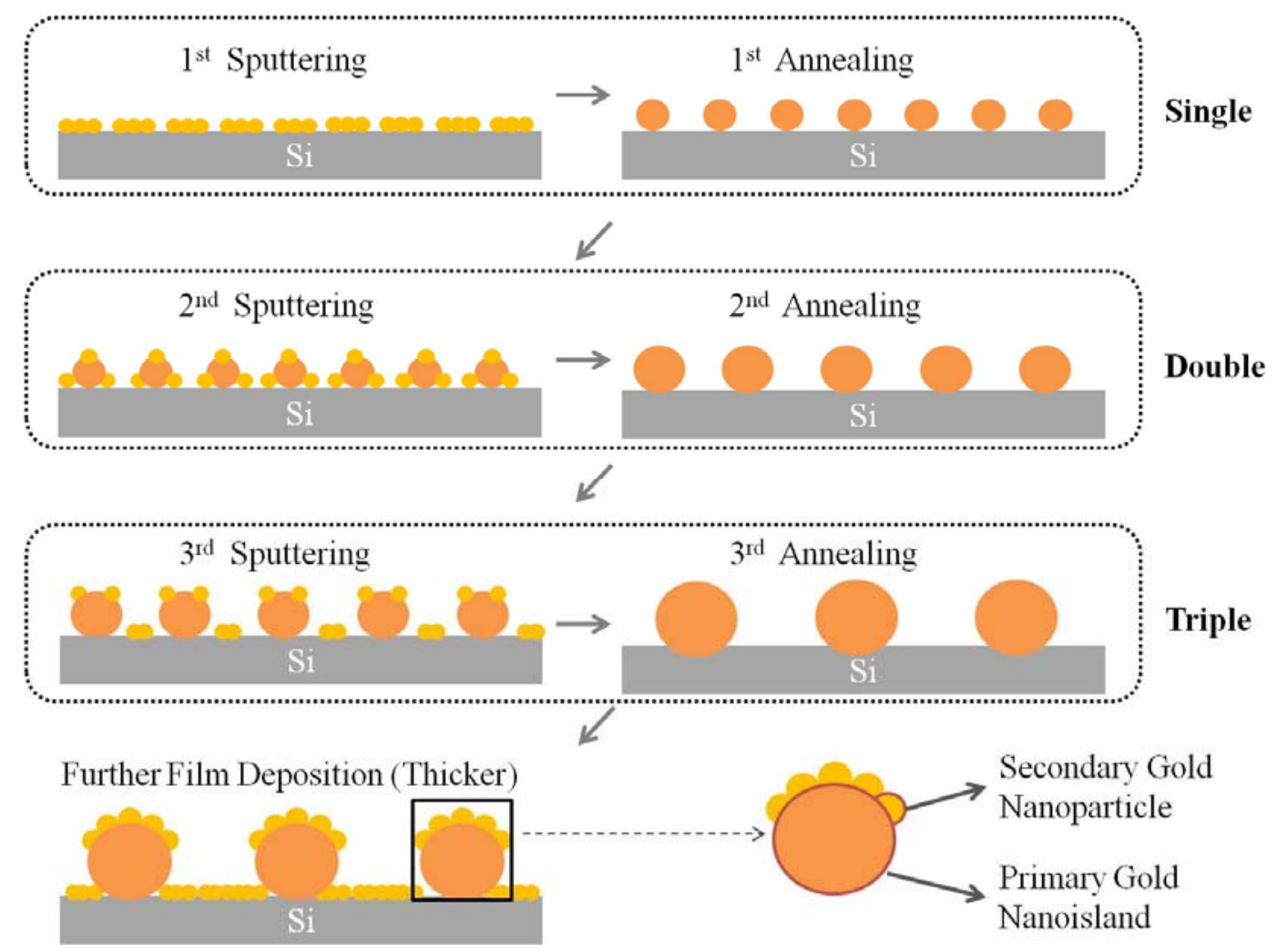

Figure 3.1. Schematic fabrication process of SERS substrates.

\subsection{Experimental section}

Si (100) wafer was rinsed by acetone, methanol, and de-ionized water sequentially, and then dried by nitrogen gas flow. An ultra thin Au film with $5 \mathrm{~nm}$ nominal thickness was sputtered on Si wafer by an Emitech K650X sputtering machine. In the present work, nominal thickness was measured by a quartz crystal balance connected with an Emitech K150 film thickness monitor. The as-coated Si wafer was annealed in a quartz tube furnace at $200{ }^{\circ} \mathrm{C}$ for $2 \mathrm{~h}$, during which 100 standard cubic centimeters per minute ( $\mathrm{sccm}$ ) mixed forming gas (95\% 
argon and $5 \%$ hydrogen) was used as the protective agent. After the $1^{\text {st }}$ annealing as shown in Fig. 3.1, typical Au nanoisland arrays had been formed. Then we repeated the $5 \mathrm{~nm}$ Au sputtering deposition and post-deposition annealing on this Au nanoisland arrays for one and two times as shown in Fig. 3.1. The three samples were referred as $A u$ nanoisland arrays with single, double, and triple sputtering deposition and post-deposition annealing processes, respectively. Then we further coated the triple processed Au nanoisland arrays with a final layer of Au thin film $(10 \mathrm{~nm}, 20 \mathrm{~nm}, 30 \mathrm{~nm}, 40 \mathrm{~nm}, 50 \mathrm{~nm}$, and $60 \mathrm{~nm}$ nominal thicknesses) with the same Emitech sputtering machine for SERS measurements. In this paper, we refer the Au nanoislands after each annealing as primary $\mathrm{Au}$ nanoislands and the final layer of $\mathrm{Au}$ deposition as secondary Au nanoparticles. Obviously, the secondary Au nanoparticles were smaller than the primary $\mathrm{Au}$ nanoislands. In addition, for the purpose of comparison, a single process of $\mathrm{Au}$ sputtering deposition and post-deposition annealing was also performed with the nominal thickness of sputtered film being set at 10 $\mathrm{nm}$ and $15 \mathrm{~nm}$. The planar morphology of Au nanoisland arrays at different stages was characterized with field emission SEM (FESEM, Hitachi S-4700), and the heights of $\mathrm{Au}$ nanoisland arrays were measured with tapping mode atomic force microscopy (AFM, Agilent 5500) coupled with VISTA T300R probe. The size distribution analysis 
of Au nanoisland arrays was carried out by measuring and counting nanoislands across $1.2 \mu \mathrm{m}$ by $1.2 \mu \mathrm{m}$ SEM images. The simulation on scattered electric field was performed with COMSOL Multiphysics.

The Raman spectrometer employed in this study was a portable system (i-Raman, B\&W Tek Inc.) with a $785 \mathrm{~nm}$ near-infrared diode laser excitation (maximum at $330 \mathrm{~mW}$ ). The Raman spectra were collected using a $20 \times$ objective with 10-second exposure time and $\sim 50$ mW laser output. Malachite green (MG) and 1,2-benzenedithiol (BDT), both from Sigma-Aldrich, were used as analytes without further purification. The analyte solutions of MG at various concentrations were prepared in acetonitrile and water mixture (1:1 in volume). For each measurement, $0.2 \mu \mathrm{l} \mathrm{MG}$ analyte solution was dropped onto a SERS substrate and dried in air. Additionally, self-assembled monolayer (SAM) of 1,2-BDT was formed on a SERS substrate in order to study its uniformity. Given the inner diameter $(5.08 \mathrm{~cm})$ of the tube used for annealing, the dimension of this SERS substrate was confined at $5 \mathrm{~cm}$ by $5 \mathrm{~cm}$. In detail, the uniformity study was started by preparing $10^{-5} \mathrm{M} 1,2-\mathrm{BDT}$ solution in 200-proof ethanol. Then the $5 \mathrm{~cm}$ by $5 \mathrm{~cm}$ SERS substrate was incubated in this solution for $2 \mathrm{~h}$. After being dried with nitrogen gas flow, the substrate was divided evenly into 100 grids ( $5 \mathrm{~mm}$ by $5 \mathrm{~mm}$ each) for SERS measurement. For each 
grid, peak intensity at band of $1030 \mathrm{~cm}^{-1}$ was recorded for uniformity analysis.

\subsection{Results and discussion}

SEM characterization shows nearly round nanoisland arrays (from top view) were formed with an average diameter of $\sim 16 \mathrm{~nm}$ after a single sputtering deposition ( $5 \mathrm{~nm}$ nominal thickness) and postdeposition annealing process (Fig. $3.2(\mathbf{a})$ corresponding to $" 1^{\text {st }}$ sputtering" and Fig. 3.2(b) to "1 $1^{\text {st }}$ annealing" in Fig. 3.1). After this process was repeated one time (Fig. $\mathbf{3 . 2 ( C )}$ corresponding to " $2^{\text {nd }}$ sputtering" and Fig. 3.2(d) to "2 ${ }^{\text {nd }}$ annealing" in Fig. 3.1) and two times (Fig. 3.2(e) corresponding to " $3^{\text {rd }}$ sputtering" and Fig. 3.2(f) to " $3^{\text {rd }}$ annealing" in Fig. 3.1), the average diameter of nanoislands grew to $\sim 24 \mathrm{~nm}$ and $\sim 38 \mathrm{~nm}$, respectively. In other words, the size of nanoisland could be controlled by adding additional process of deposition and annealing, which is evidenced by the histogram in Fig. 3.3 as well. It is also worthwhile to mention that the density of nanoisland declined (values shown in Fig. 3.3) as the nanoislands grew bigger, indicative of merging among adjacent nanoislands during the subsequent annealing process. We speculate this merging effect, along with the new sputtered $\mathrm{Au}$, contributed to the growth of nanoislands. 

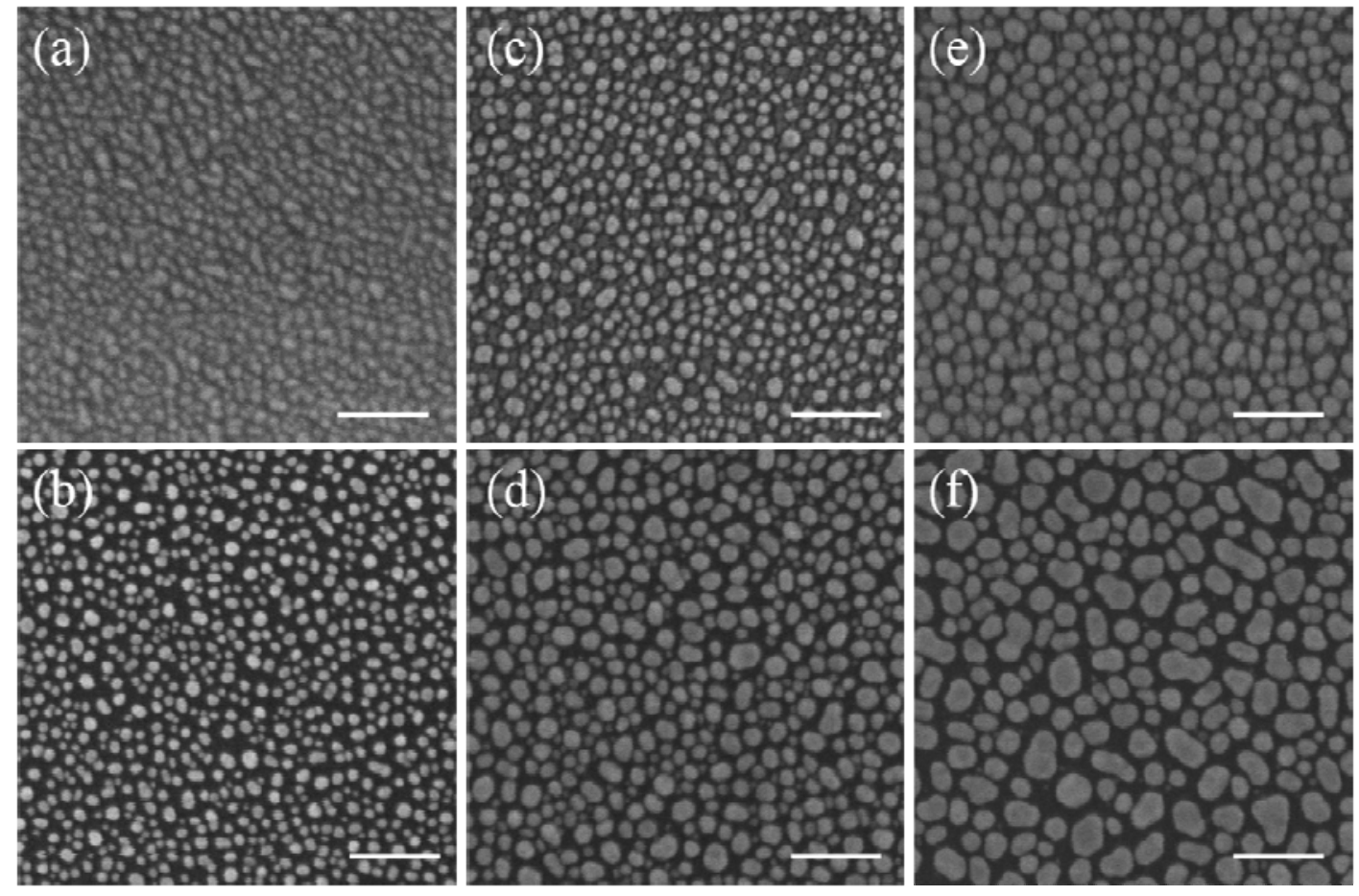

Figure 3.2. SEM images of Au nanoisland arrays at different stages during growth. Morphology of nanoisland arrays after $1^{\text {st }}(a), 2^{\text {nd }}(c)$, and $3^{\text {rd }}(e)$ sputtering. Morphology of nanoisland arrays after $1^{\text {st }}(b), 2^{\text {nd }}(d)$, and $3^{\text {rd }}(f)$ annealing. Alternatively, (b), (d), and (f) represent single, double, and triple processed Au nanoisland arrays, respectively. All scale bars equal to $200 \mathrm{~nm}$.

In addition, the height information acquired by AFM measurement (Fig. 3.4) confirms the vertical growth of nanoislands with repeated deposition and annealing processes. Specifically, the average heights of single, double, and triple processed nanoisland arrays were $\sim 11 \mathrm{~nm}, \sim 15 \mathrm{~nm}$, and $\sim 24 \mathrm{~nm}$, respectively. Apparently, the heights of the nanoislands were smaller than their corresponding planar diameters in all three samples. On one hand, it is quite possible that the nanoislands were indeed shaped like pancakes, as hinted by the SEM and AFM measurements, considering the nanoislands were 


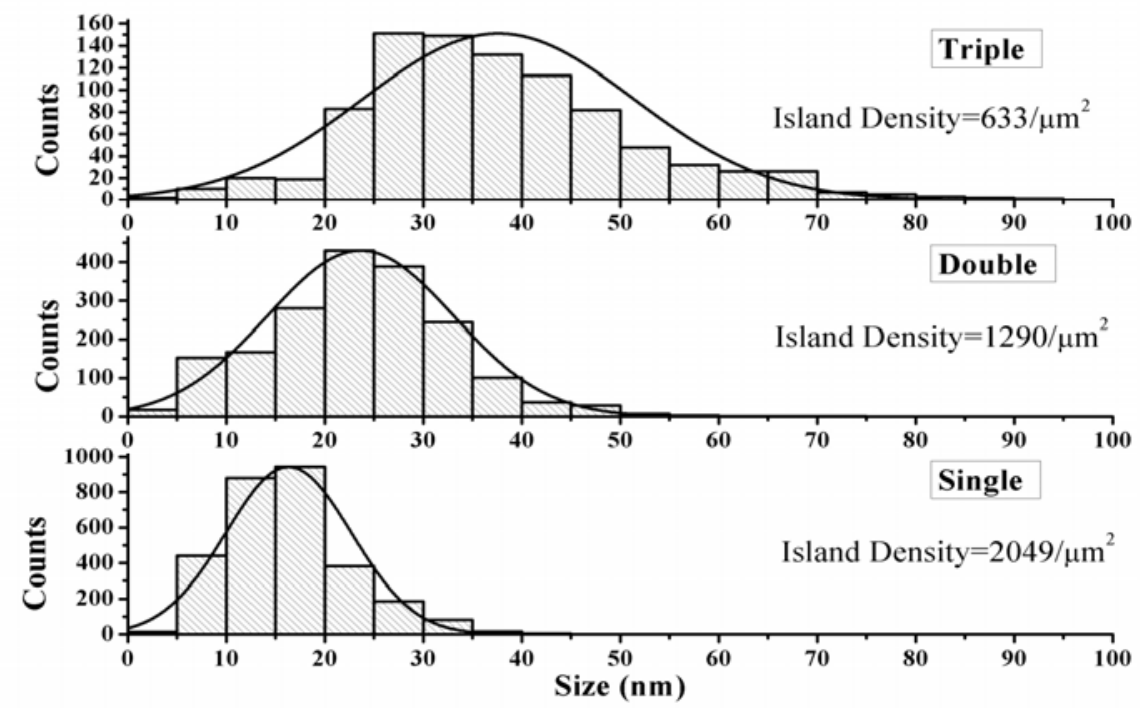

Figure 3.3. Histogram of nanoislands size distribution after single, double, and triple deposition and annealing processes.

originated from a thin film. On the other hand, it is also likely that the pancake shape resulted from AFM artifacts. That is, considering the tip radius (10 $\mathrm{nm}$ ) of AFM probe was comparable to the width of gaps between nanoislands, the probe hardly touched the bottom of the gaps, leaving actificailly raised baseline of AFM images and, in turn, reduced heights of nanoislands.

By contrast, a single process of sputtering deposition and postdeposition annealing was also applied on samples with initial nominal thicknesses of $10 \mathrm{~nm}$ (equivalent to $2 \times 5 \mathrm{~nm}$ ) and $15 \mathrm{~nm}$ (equivalent to $3 \times 5 \mathrm{~nm})$. The planar morphology of these two thin films before annealing is shown in Fig. 3.5(a) and (b), respectively. After annealing, Fig. 3.5(c) and (d) depict that the resulted structures 
were larger, non-uniform, and irregular in shape. This comparison justified our efforts of adopting repeated deposition and annealing processes in order to achieve large nanoisland arrays with excellent uniformity and shape control.

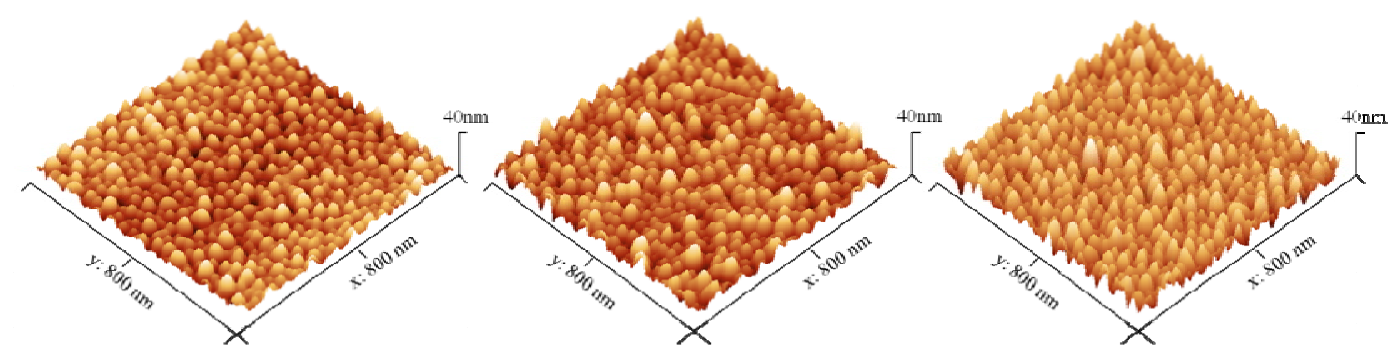

Figure 3.4. AFM images of single (left), double (middle), and triple (right) processed Au nanoisland arrays. 

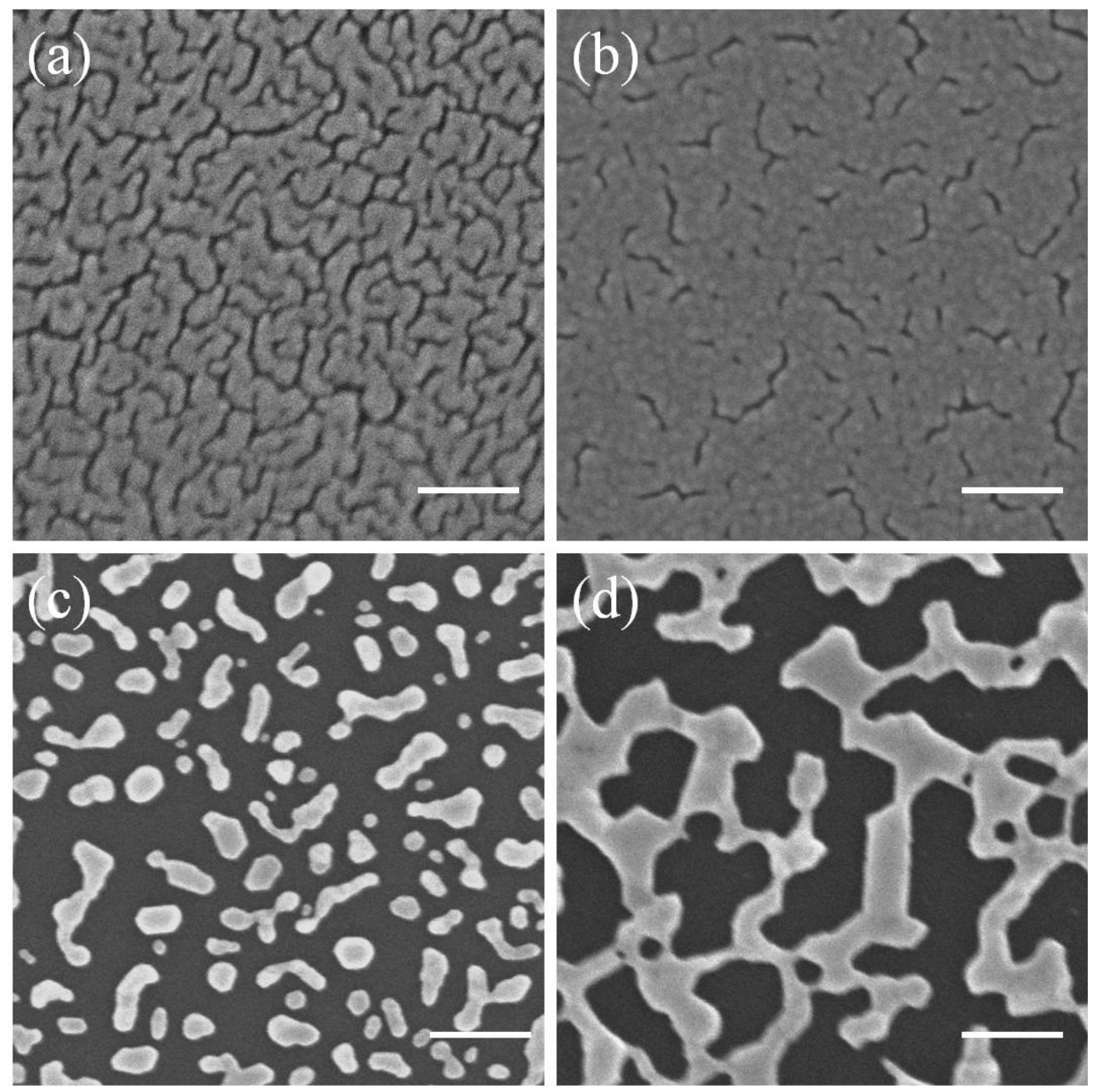

Figure 3.5. SEM images of $10 \mathrm{~nm}$ and $15 \mathrm{~nm}$ Au thin films before and after annealing on Si substrate. (a) $10 \mathrm{~nm}$ before annealing; (b) $15 \mathrm{~nm}$ before annealing; (c) $10 \mathrm{~nm}$ after annealing; (d) $15 \mathrm{~nm}$ after annealing. All scale bars equal to $200 \mathrm{~nm}$.

In essence, sputtered $\mathrm{Au}$ is composed of tiny nanoparticles with diameter of a few nanometers. Au sputtered onto substrates evolves to completely covered thin film state through a series of stages, namely isolated islands, percolation, holes filling, and finally thin film [2]. When induced by annealing, the sputtered Au dramatically 
transforms into larger islands with different shapes. This process is usually referred as solid-state dewetting. The reduction of surface free energy (through the reduction of surface area) and the difference in thermal expansion coefficient between $\mathrm{Au}$ and substrate are considered as the driving forces for the morphology change [18]. Specifically, inhomogeneous stress distribution at the Au-Si interface promotes the migration of $\mathrm{Au}$ atoms to more relaxed regions. In addition, elevated temperatures enhance the mobility of Au atoms and facilitate small Au nanoparticles coalescing into larger islands to reduce the surface free energy [25]. In general, the morphology of resulted islands is determined by two factors. One is the initial film thickness or the initial stage of the sputtered Au (isolated islands, percolation, or holes filling) [26]. The other one is the annealing condition, including temperature, atmosphere, and time [27-29]. In our study, it seems that $5 \mathrm{~nm} \mathrm{Au}$ deposition was at the stage of isolated islands (Fig. 3.2(a)), $10 \mathrm{~nm}$ Au deposition was at the stage of percolation (Fig. 3.5(a)), and $15 \mathrm{~nm} \mathrm{Au} \mathrm{deposition} \mathrm{was} \mathrm{at} \mathrm{the} \mathrm{stage} \mathrm{of} \mathrm{holes} \mathrm{filling} \mathrm{(Fig.}$ 3.5(b)). Since the annealing condition was kept unchanged in our experiments, the initial film thickness apparently accounted for the morphological variation of Au nanoislands we observed in Fig. 3.2(b), Fig. 3.5(c), and Fig. 3.5(d). 
Size and spacing of Au nanoparticles strongly affect localized surface plasmon resonance (LSPR) $[30,31]$ which constitutes the foundation of electromagnetic enhancement of SERS [32-34]. With interparticle coupling effect being neglected, it was demonstrated both theoretically [35] and experimentally [36] that an isolated spherical Au nanoparticle yielded the maximum enhancement when particle diameter was $\sim 60 \mathrm{~nm}$. Before reaching to the maximum, the enhancement climbed with the increasing particle size. Regarding to spacing, with particle diameter being fixed, Zhu et al. [37] observed that interparticle coupling effect started to take place when the particle center to center distance was less than twice of particle diameter, and the effect then rose up dramatically with the decreasing interparticle distance. In other words, once the interparticle coupling took effect, the enhancement increased sharply with the increasing ratio of particle diameter to interparticle distance. Although smaller interparticle distance generally leads to higher enhancement, the chances for molecules to locate in such "hot spot" zone may also decrease as the spacing between particles decreases. As a result, there might be an optimal interparticle distance, not necessarily the smallest, that provides the highest enhancement for the substrates. In this study, we carried out the first SERS measurement on as-grown primary Au nanoisland arrays. Figure 3.6 shows the SERS spectra of 2 parts per 
million (ppm) MG on these single, double, and triple deposition and annealing processed substrates. As indicated in the spectra, triple processed sample exhibited the highest enhancement, which was consistent with others' works [30, 31, 35-37]. In details, as the process was repeated, the nanoisland diameter was increasing towards $60 \mathrm{~nm}$, the preferred size for maximum SERS performance when not considering particle interactions; meanwhile, the nanoisland diameter to interisland distance ratio was getting higher as well (Fig. 3.2(b), (d), and (f)). Both these two factors suggested triple processed

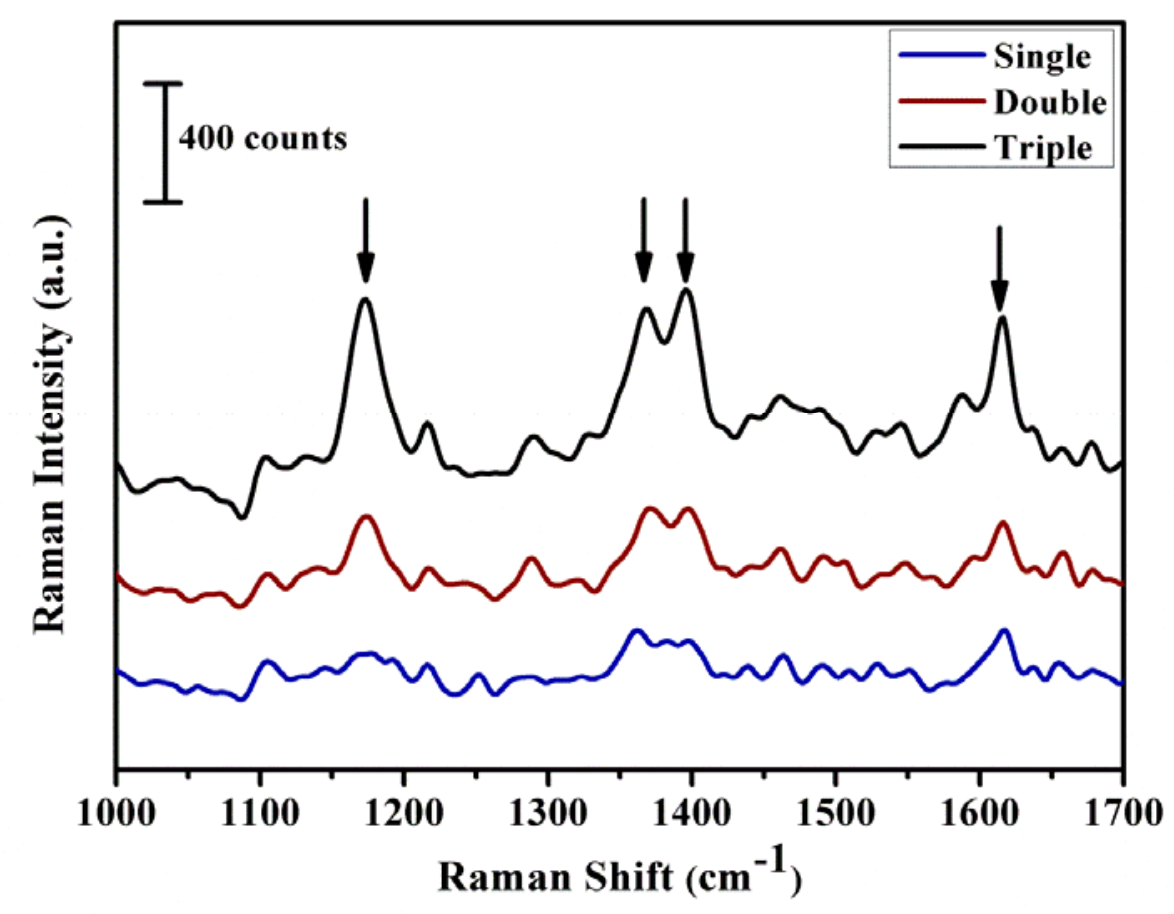

Figure 3.6. SERS spectra of $2 \mathrm{ppm} \mathrm{MG}$ on single, double, and triple processed $\mathrm{Au}$ nanoisland arrays. 
substrate the best one for SERS purpose.

In an attempt to produce better SERS effect, a series of Au thin films $(10 \mathrm{~nm}, 20 \mathrm{~nm}, 30 \mathrm{~nm}, 40 \mathrm{~nm}, 50 \mathrm{~nm}$, and $60 \mathrm{~nm}$ nominal thicknesses) were deposited over the primary Au nanoisland arrays as such thin film is actually made of nanoparticles in a few nanometers. For this study, we selected nanoisland arrays with triple deposition and annealing process, which generated the best performance prior to the final layer of $\mathrm{Au}$ thin film deposition. In order to investigate the influence of the thin film thickness on SERS performance, these substrates were tested by measuring 200 parts per billion (ppb) MG with all other experimental conditions being equal. Figure 3.7(a) shows that the Raman signal was initially increasing as the film became thicker and then started to decrease when the film was thicker than $40 \mathrm{~nm}$. To confirm it, a quantitative analysis was performed by normalizing the Raman intensity (peak height) at band of $1173 \mathrm{~cm}^{-1}$. In details, the peak height of the $40 \mathrm{~nm}$ sample was set as $I_{\max }$ and the normalized Raman intensity was defined by dividing all peak heights by $I_{\max }$. The relationship between film thickness and normalized Raman intensity $\left(\mathrm{I} / \mathrm{I}_{\max }\right)$ is presented in Fig. 3.7(b). The graph further suggests the optimal thickness residing at $\sim 40 \mathrm{~nm}$. We speculate that the final layer of $\mathrm{Au}$ thin film exerted two opposite effects on SERS performance. One of them was to boost the SERS 
performance with (i) the improved surface roughness at a few nanometer scale through the addition of secondary Au nanoparticles and (ii) the decrease of spacing between primary Au nanoislands with relatively thin Au coating. The other effect was to degrade the SERS performance with the reduction of roughness at tens of nanometer scale as the gaps between primary Au nanoislands were closed up with thicker Au coating. The following two paragraphs elaborate the two opposite effects with the support of SEM images and additional
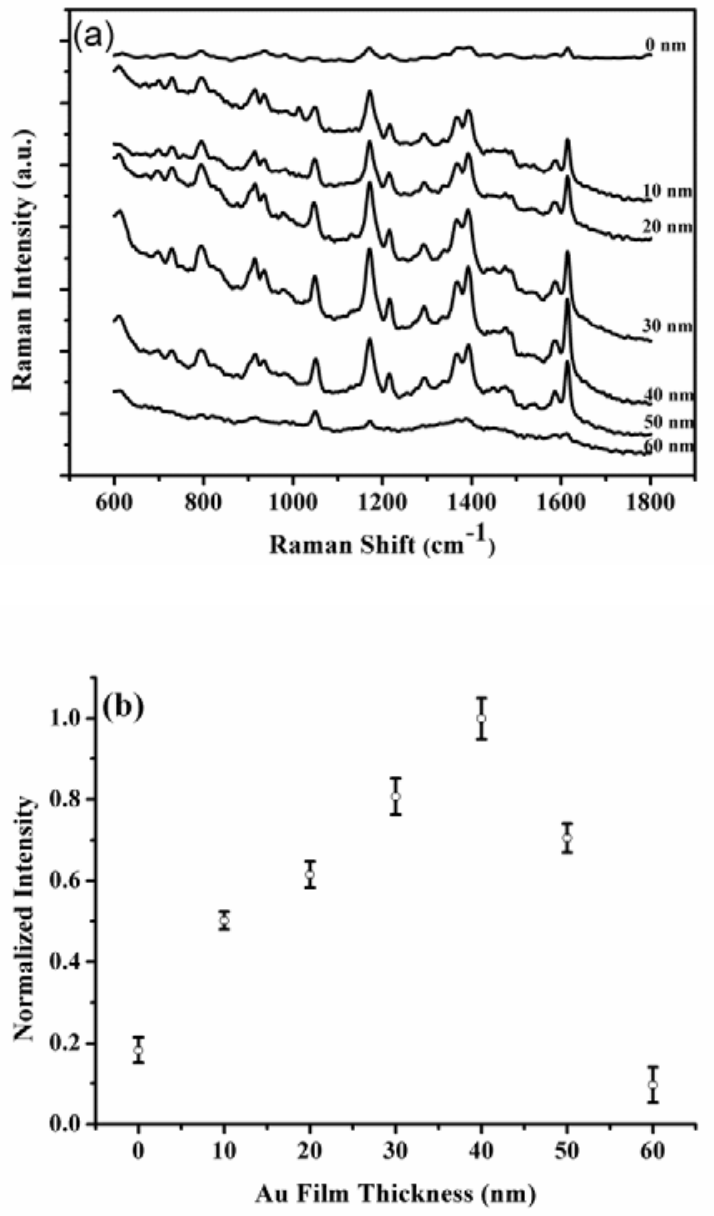

Figure 3.7. (a) SERS spectra of 200 ppb MG on triple processed Au nanoislands arrays with $0 \mathrm{~nm}$, $10 \mathrm{~nm}, 20 \mathrm{~nm}, 30 \mathrm{~nm}, 40 \mathrm{~nm}, 50$ $\mathrm{nm}$, and $60 \mathrm{~nm}$ gold depositions. (b) Relationship between normalized intensity at $v=1173$ $\mathrm{cm}^{-1}$ and Au deposition thickness. 
analysis.

First, the individual primary nanoisland was examined. As shown in Fig. 3.8(a-c), the surface of primary nanoisland was relatively clean and smooth after $20 \mathrm{~nm} \mathrm{Au} \mathrm{deposition,} \mathrm{and} \mathrm{was} \mathrm{then} \mathrm{getting}$ rougher after $40 \mathrm{~nm}$ and $60 \mathrm{~nm}$ Au depositions. In other words, the $\mathrm{Au}$ deposition gradually introduced small secondary nanoparticles on top of the primary nanoislands, resulting in the addition of roughness at a few nanometer scale. To find out the effect produced by these secondary nanoparticles, finite element method simulation of scattered electric field with a vertical incident plane wave $(\lambda=785 \mathrm{~nm})$ was carried out on two equally sized objects: a smooth sphere and a secondary nanoparticle-coated sphere. Figure $\mathbf{3 . 8 ( d )}$ and (e) resulted from the simulation demonstrate that the latter with additional roughness induced more intense scattered electric field close to sphere surface, which suggested secondary nanoparticle-coated nanoislands produced higher Raman enhancement. This result was in good agreement with other researchers' conclusion as they found out spheres with rough surface textures (at a few nanometer scale) displayed higher SERS sensitivity [38-40]. In summary, the simulation on individual primary nanoisland favored $40 \mathrm{~nm}$ and $60 \mathrm{~nm}$ films for superior SERS performance. 

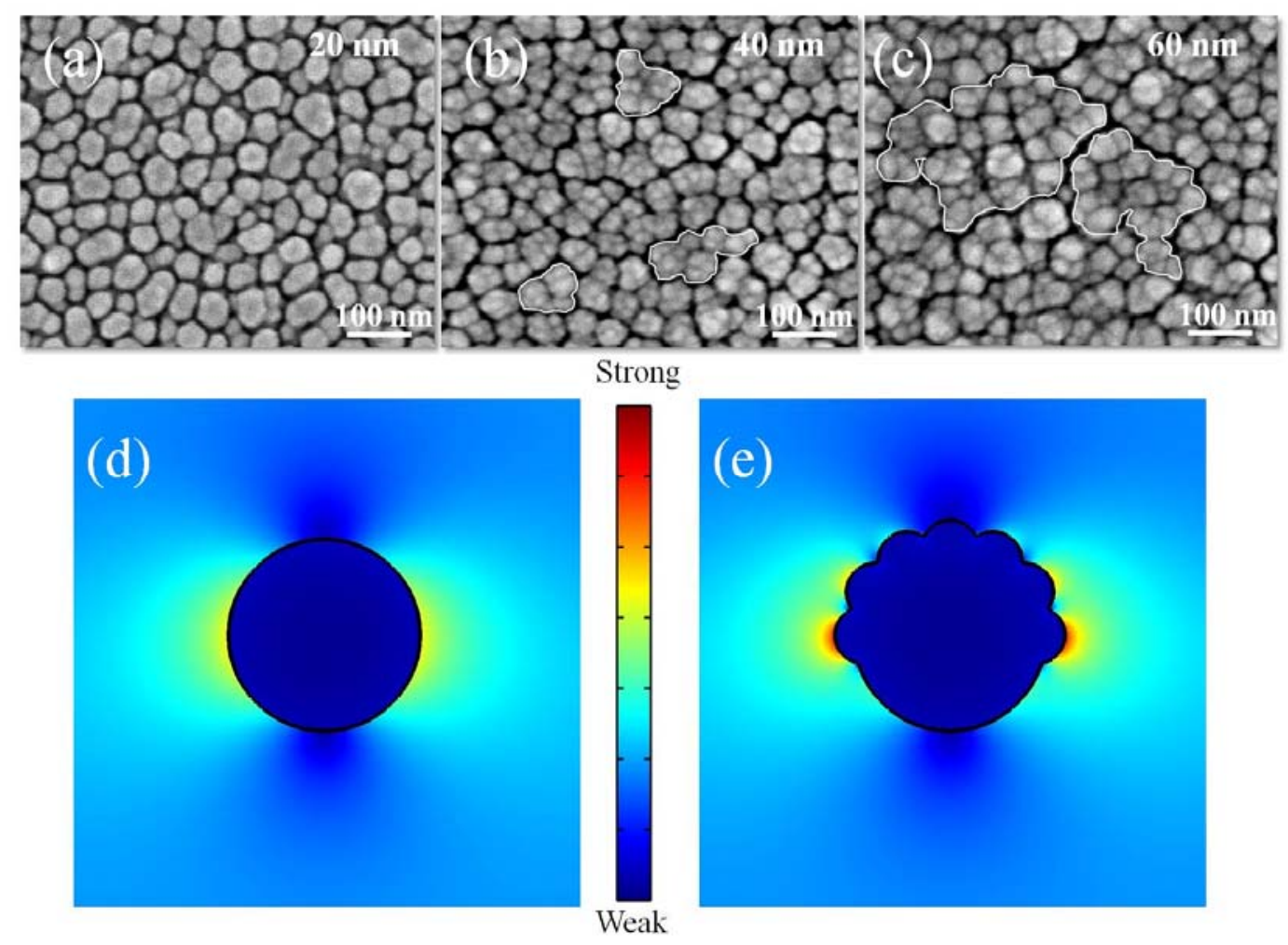

Figure 3.8. SEM images of triple processed Au nanoisland arrays with $20 \mathrm{~nm}$ (a), $40 \mathrm{~nm}$ (b), and $60 \mathrm{~nm}$ (c) Au depositions, respectively. Marks in (b) and (c) show the formation of bigger islands. Simulation of scattered electric field induced by a vertical incident plane wave $(\lambda=785 \mathrm{~nm})$ on a smooth sphere (d) and a secondary nanoparticle-coated sphere (e).

Second, according to previous studies [41-43], gaps between adjacent nanoparticles are believed to be the region where the highest enhancement is present and thus often referred as SERS "hot spots". As shown in Fig. 3.8(a), the primary nanoislands remained separated from each other, and, in turn, lots of gaps were present after $20 \mathrm{~nm}$ Au deposition. In addition, this layer of Au deposition made the gaps narrower, which ultimately helped to improve the SERS performance due to a higher ratio of particle diameter to interparticle distance. 
When the film thickness reached to $40 \mathrm{~nm}$ and then $60 \mathrm{~nm}$, however, the gaps gradually disappeared as they were filled by the Au deposition. As marked in Fig. 3.8(b) and (c), some initially isolated primary nanoislands formed into bigger nanoislands. As a consequence, the density of so called "hot spots" declined due to the loss of gaps, and the SERS performance was adversely affected.

Based on such analysis, there should be a compromise to balance the positive and negative effects of the final layer $\mathrm{Au}$ deposition. In fact, the SERS measurement in Fig. 3.7 agrees upon the existence of such compromising behavior in that the optimal thickness resides in between too thin and too thick coatings.

In order to evaluate the sensitivity of this SERS substrate, a series of low level MG analytes were tested. Triple processed Au nanoisland arrays with $40 \mathrm{~nm}$ Au deposition were used for the sensitivity analysis. Figure $\mathbf{3 . 9 ( a )}$ exhibits the stacking spectra of these MG analytes, along with a blank scan for reference. As shown, the responses at bands of $1173 \mathrm{~cm}^{-1}$ and $1615 \mathrm{~cm}^{-1}$ were still observable at the concentration of as low as $20 \mathrm{ppb}$. Moreover, the relationship between Raman intensity $\left(1615 \mathrm{~cm}^{-1}\right)$ and analyte concentration was plotted in Fig. 3.9(b). For each concentration, five spectra were collected and the corresponding peak heights were 
recorded. The well fitted straight line in Fig. 3.9(b) demonstrates the linear behavior $\left(R^{2}=0.9903\right)$ of this substrate, suggesting good potential for quantitative SERS analysis.

In addition, the analytical enhancement factor (AEF) of the best sample (triple processed nanoisland arrays with $40 \mathrm{~nm}$ Au coating) was studied to quantify its sensitivity. AEF has already been defined in Chapter 2. In this case, as shown in Fig. 3.10, $C_{S E R S}$ and $c_{R}$ are $10^{-5} \mathrm{M}$

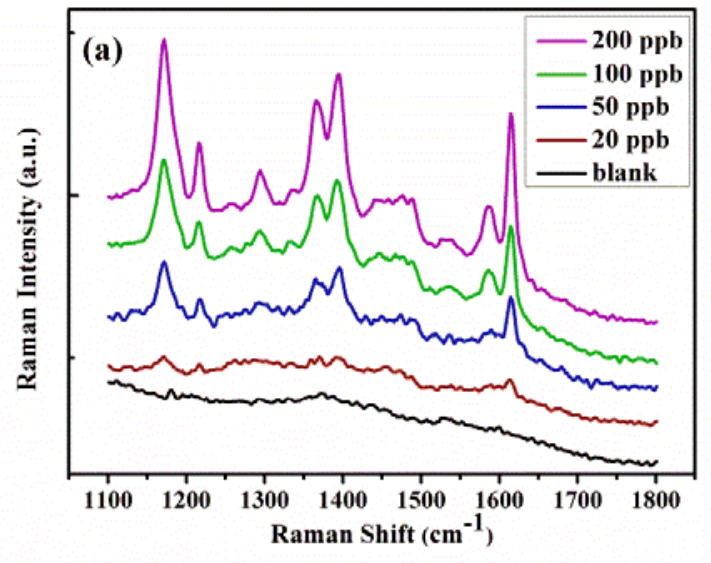

Figure 3.9. (a) SERS spectra of a series of low level MGs and a blank reference collected on a triple processed $\mathrm{Au}$ nanoisland arrays with 40 $\mathrm{nm}$ Au deposition. (b) Relationship between Raman intensity at $v=1615 \mathrm{~cm}^{-1}$ and concentration of analyte solution.

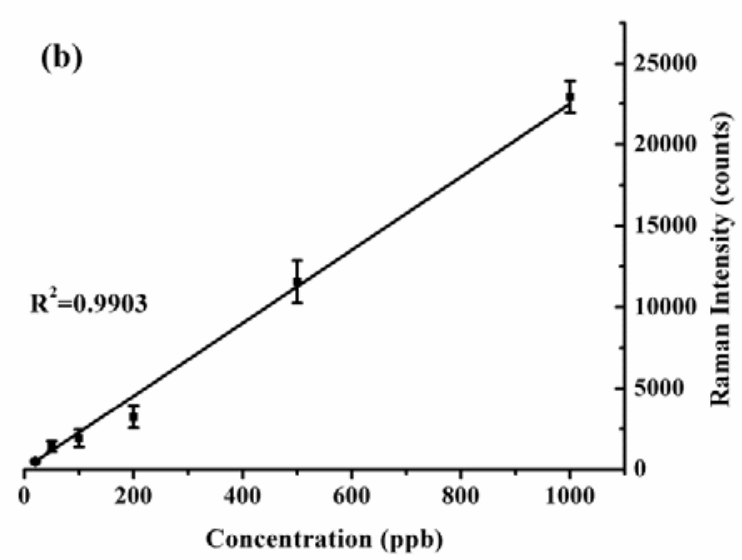


and $10^{-1} \mathrm{M}$ BDT, respectively, while Ramen intensities at $\mathrm{v}=1030 \mathrm{~cm}^{-1}$ $I_{\text {SERS }}$ and $I_{R}$ are about 1000 and 350, respectively. Hence, this SERS substrate is projected to have an AEF of $3 \times 10^{4}$ over $50 \mathrm{~nm}$ bare $\mathrm{Au}$ film.

In practical application, uniformity of SERS substrate is as important as enhancement factor and sometimes is even considered as the most important parameter of SERS substrates. According to previous studies [44-46], enhancement factor of $10^{6}-10^{9}$ is sufficiently high for single molecule detection. Thus, research on the subject of

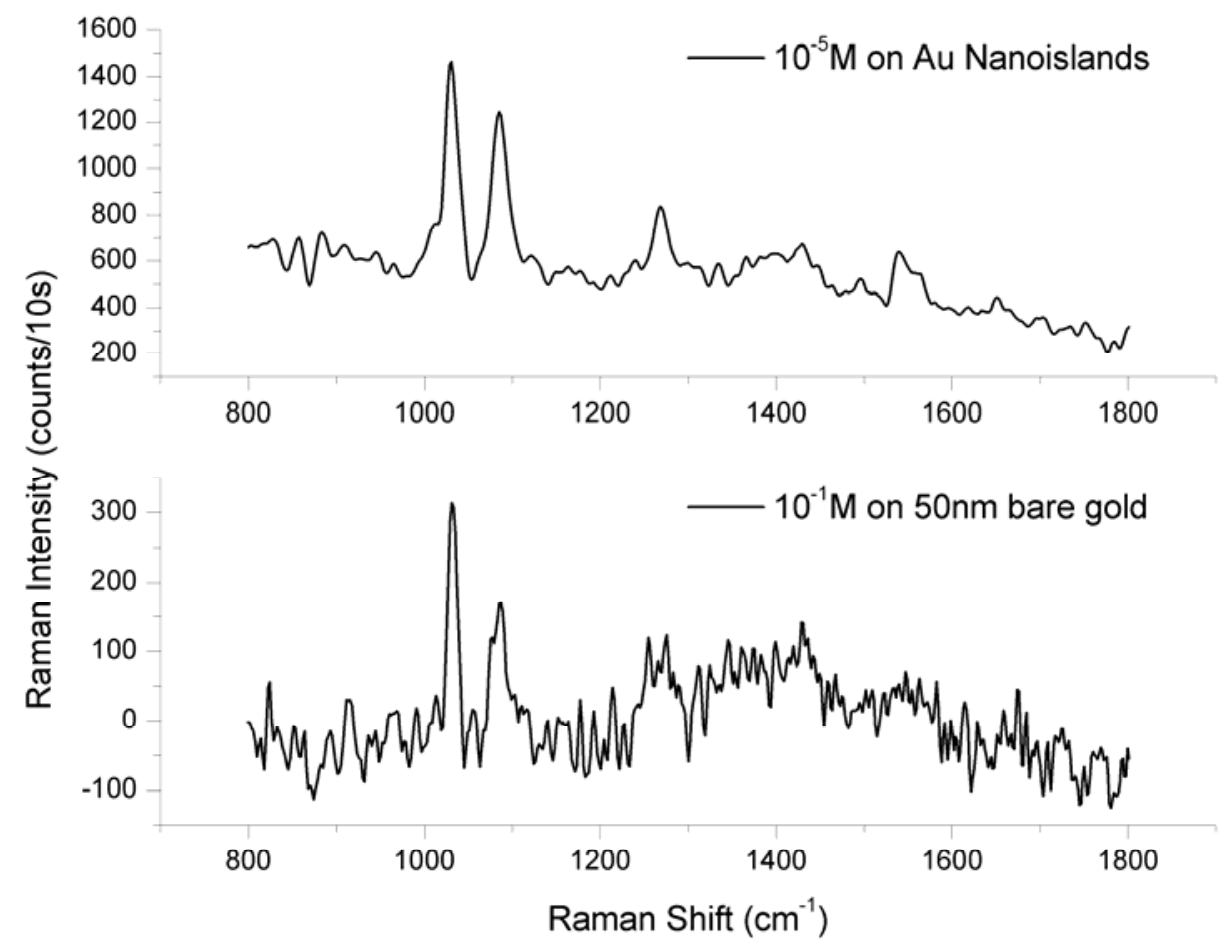

Figure 3.10. SERS spectrum of $10^{-5} \mathrm{M}$ BDT acquired on triple processed nanoisland arrays with $40 \mathrm{~nm}$ Au coating (top). Raman spectrum of $10^{-1} \mathrm{M}$ BDT acquired on $50 \mathrm{~nm}$ Au film on un-etched Si (bottom). 
uniformity has become more valuable and meaningful. In the present work, BDT was used to investigate the uniformity of our SERS substrate since BDT was expected to form monolayer on Au surface. Under such circumstances, aggregation or poor distribution of analyte could be precluded and the results would more likely reflect the intrinsic property of substrate. The SERS substrate used for uniformity study was triple processed Au nanoisland arrays with $40 \mathrm{~nm}$ Au deposition. The dimension of this SERS substrate was set at $5 \mathrm{~cm}$ by 5 $\mathrm{cm}$ and this dimension was the upper limit we were able to reach because the tube used for annealing has an inner diameter of $5.08 \mathrm{~cm}$. Considering the fact that $5 \mathrm{~cm}$ by $5 \mathrm{~cm}$ dimension was too large to obtain very uniform deposition by our sputtering machine, we purposely placed the bottom-left corner (the origin in Fig. 3.11) of this substrate at the center of sample stage during all sputtering processes. With such setup, we could to a certain degree track the deposition variation over the entire substrate. After the formation of self assembled BDT monolayer, the $5 \mathrm{~cm}$ by $5 \mathrm{~cm}$ SERS substrate was divided evenly into 100 grids ( $5 \mathrm{~mm}$ by $5 \mathrm{~mm}$ each). The intensity of peak at band of $1030 \mathrm{~cm}^{-1}$ from each grid was collected and plotted versus substrate's $X-Y$ position, shown in Fig. 3.11. The relative standard deviation (RSD) of Raman intensity was estimated to be $\sim 7 \%$ over 100 grids. Please note that the intensities of SERS signals around 
all corners of the square substrate, except for bottom-left, were lower than those at the rest area. This phenomenon may be due to the deposition variation generated by our sputtering machine whose deposition rate is higher at center and lower at edges. Lastly, though 5 $\mathrm{cm}$ by $5 \mathrm{~cm}$ was the upper limit to us, this SERS substrate is highly likely to be further scaled up with large capacity equipments.

\subsection{Conclusion}

In this chapter, Au nanoisland arrays with well controlled growth

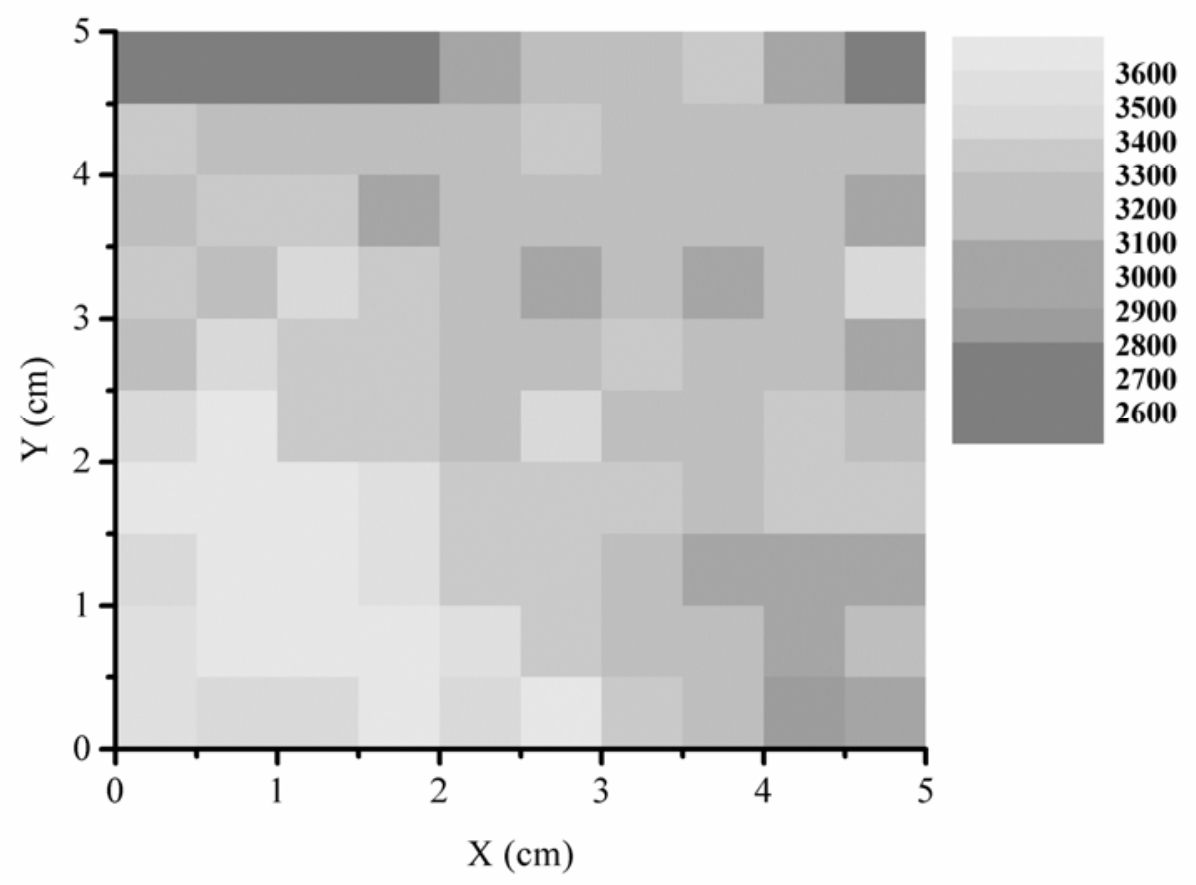

Figure 3.11. The distribution of Raman intensity $\left(v=1030 \mathrm{~cm}^{-1}\right.$ of $\left.10^{-4} \mathrm{M} B D T\right)$ over a $5 \mathrm{~cm}$ by $5 \mathrm{~cm}$ SERS substrate (same recipe as the one in Fig. 3.9) divided with 100 equal-sized grids. 
were achieved by repeated sputtering deposition and post-deposition annealing processes. SEM and AFM characterizations of the Au nanoisland arrays at different stages revealed the evolution of nanoisland structure and demonstrated the superiority of repeated processes for fabricating large Au nanoisland arrays with excellent uniformity. SERS testing on single, double, and triple processed Au nanoisland arrays confirmed the necessity of pursuing nanoislands with larger size as the triple processed sample exhibited the highest SERS enhancement among the three primary Au nanoisland arrays. By further sputtering the triple processed sample with a final layer of $\mathrm{Au}$ thin film, we were able to obtain the optimized SERS substrate with an analytical enhancement factor (AEF) as high as $3 \times 10^{4}$. In addition, the positive and negative effects of the final layer Au film were investigated and their compromising behavior was demonstrated. In the end, we studied the uniformity of this SERS substrate on a $5 \mathrm{~cm}$ by $5 \mathrm{~cm}$ platform. SERS measurement showed a RSD of $\sim 7 \%$ in terms of spectral variation over the entire substrate, suggesting the approach in the present work is promising for large scale fabrication of SERS substrates with high enhancement factors and good uniformity while at low cost.

\subsection{Reference}


1. Royer, P., Goudonnet, J. P., Warmack, R. J. and Ferrell, T. L., Substrate effects on surface-plasmon spectra in metal-island films, Physical Review B, 1987, 35, p. 3753-3759.

2. Yu, X., Duxbury, P. M., Jeffers, G. and Dubson, M. A., Coalescence and percolation in thin metal films, Physical Review B, 1991, 44, p. 13163-13166.

3. Kaiser, N., Review of the Fundamentals of Thin-Film Growth, Applied Optics, 2002, 41, p. 3053-3060.

4. Xu, G., Tazawa, M., Jin, P. and Nakao, S., Surface plasmon resonance of sputtered Ag films: substrate and mass thickness dependence, Applied Physics A, 2005, 80, p. 1535-1540.

5. Aslan, K., Leonenko, Z., Lakowicz, J. and Geddes, C., Annealed Silver-Island Films for Applications in Metal-Enhanced Fluorescence: Interpretation in Terms of Radiating Plasmons, Journal of Fluorescence, 2005, 15, p. 643-654.

6. Hung, W.-C., Cheng, W.-H., Lin, Y.-S., Jang, D.-J., Jiang, I. M. and Tsai, M.S., Surface plasmons induced extra diffraction band of cholesteric liquid crystal grating, Journal of Applied Physics, 2008, 104, p. 063106-5.

7. Leitner, A., Zhao, Z., Brunner, H., Aussenegg, F. R. and Wokaun, A., Optical properties of a metal island film close to a smooth metal surface, Applied Optics, 1993, 32, p. 102-110.

8. Yamaguchi, T., Takahashi, H. and Sudoh, A., Optical behavior of a metal island film, Journal of the Optical Society of America, 1978, 68, p. 1039-1044.

9. Jensen, T. R., Duyne, R. P. V., Johnson, S. A. and Maroni, V. A., SurfaceEnhanced Infrared Spectroscopy: A Comparison of Metal Island Films with Discrete and Nondiscrete Surface Plasmons, Applied Spectroscopy, 2000, 54, p. 371-377.

10. Pavaskar, P., Hsu, I. K., Theiss, J., Hung, W. H. and Cronin, S. B., A microscopic study of strongly plasmonic $A u$ and $A g$ island thin films, Journal of Applied Physics, 2013, 113, p. 034302-6.

11. Whitney, A. V., Van Duyne, R. P. and Casadio, F. Silver island films as substrate for surface-enhanced Raman spectroscopy (SERS): a methodological study on their application to artists' red dyestuffs. 2005. 
12. Félidj, N., Aubard, J., Lévi, G., Krenn, J. R., Salerno, M., Schider, G., Lamprecht, B., Leitner, A. and Aussenegg, F. R., Controlling the optical response of regular arrays of gold particles for surface-enhanced Raman scattering, Physical Review B, 2002, 65, p. 075419.

13. Cialla, D., Hübner, U., Schneidewind, H., Möller, R. and Popp, J., Probing Innovative Microfabricated Substrates for their Reproducible SERS Activity, ChemPhysChem, 2008, 9, p. 758-762.

14. Haynes, C. L. and Van Duyne, R. P., Nanosphere Lithography: A Versatile Nanofabrication Tool for Studies of Size-Dependent Nanoparticle Optics, Journal of Physical Chemistry B, 2001, 105, p. 5599-5611.

15. Lin, T.-H., Linn, N. C., Tarajano, L., Jiang, B. and Jiang, P., Electrochemical SERS at Periodic Metallic Nanopyramid Arrays, Journal of Physical Chemistry C, 2009, 113, p. 1367-1372.

16. Lee, W., Lee, S. Y., Briber, R. M. and Rabin, O., Self-Assembled SERS Substrates with Tunable Surface Plasmon Resonances, Advanced Functional Materials, 2011, 21, p. 3424-3429.

17. Galopin, E., Noual, A., Niedziółka-Jönsson, J., Jönsson-Niedziółka, M., Akjouj, A., Pennec, Y., Djafari-Rouhani, B., Boukherroub, R. and Szunerits, S., Shortand Long-Range Sensing Using Plasmonic Nanostrucures: Experimental and Theoretical Studies, Journal of Physical Chemistry C, 2009, 113, p. 1592115927.

18. Serrano, A., de la Fuente, O. R. and Garcia, M. A., Extended and localized surface plasmons in annealed Au films on glass substrates, Journal of Applied Physics, 2010, 108, p. 074303-7.

19. Gupta, R., Dyer, M. J. and Weimer, W. A., Preparation and characterization of surface plasmon resonance tunable gold and silver films, Journal of Applied Physics, 2002, 92, p. 5264-5271.

20. Luo, Y., Ruff, J., Ray, R., Gu, Y., Ploehn, H. J. and Scrivens, W. A., VaporAssisted Remodeling of Thin Gold Films, Chemistry of Materials, 2005, 17, p. 5014-5023.

21. Kalyuzhny, G., Vaskevich, A., Schneeweiss, M. A. and Rubinstein, I., Transmission Surface-Plasmon Resonance (T-SPR) Measurements for Monitoring Adsorption on Ultrathin Gold Island Films, Chemistry - A European Journal, 2002, 8, p. 3849-3857. 
22. Sabine, S., Vera, G. P., Mael, M. and Rabah, B., Gold island films on indium tin oxide for localized surface plasmon sensing, Nanotechnology, 2008, 19, p. 195712.

23. Warmack, R. J., Ferrell, T. L. and Becker, R. S., Scanning tunneling microscopy of silver and gold islands on silicon, Physica Scripta, 1988, 38, p. 159.

24. Van Duyne, R. P., Hulteen, J. C. and Treichel, D. A., Atomic force microscopy and surface-enhanced Raman spectroscopy. I. Ag island films and Ag film over polymer nanosphere surfaces supported on glass, The Journal of Chemical Physics, 1993, 99, p. 2101-2115.

25. Doron-Mor, I., Barkay, Z., Filip-Granit, N., Vaskevich, A. and Rubinstein, I., Ultrathin Gold Island Films on Silanized Glass. Morphology and Optical Properties, Chemistry of Materials, 2004, 16, p. 3476-3483.

26. Tesler, A. B., Chuntonov, L., Karakouz, T., Bendikov, T. A., Haran, G., Vaskevich, A. and Rubinstein, I., Tunable Localized Plasmon Transducers Prepared by Thermal Dewetting of Percolated Evaporated Gold Films, Journal of Physical Chemistry C, 2011, 115, p. 24642-24652.

27. Karakouz, T., Holder, D., Goomanovsky, M., Vaskevich, A. and Rubinstein, I., Morphology and Refractive Index Sensitivity of Gold Island Films, Chemistry of Materials, 2009, 21, p. 5875-5885.

28. Roark, S. E. and Rowlen, K. L., Thin silver films: influence of substrate and postdeposition treatment on morphology and optical properties, Anal Chem, 1994,66 , p. 261-270.

29. Presland, A. E. B., Price, G. L. and Trimm, D. L., Hillock formation by surface diffusion on thin silver films, Surface Science, 1972, 29, p. 424-434.

30. Fang, P.-P., Li, J.-F., Yang, Z.-L., Li, L.-M., Ren, B. and Tian, Z.-Q., Optimization of SERS activities of gold nanoparticles and gold-core-palladiumshell nanoparticles by controlling size and shell thickness, Journal of Raman Spectroscopy, 2008, 39, p. 1679-1687.

31. Njoki, P. N., Lim, I. I. S., Mott, D., Park, H.-Y., Khan, B., Mishra, S., Sujakumar, R., Luo, J. and Zhong, C.-J., Size Correlation of Optical and Spectroscopic Properties for Gold Nanoparticles, Journal of Physical Chemistry C, 2007, 111, p. 14664-14669. 
32. Jeanmaire, D. L. and Van Duyne, R. P., Surface raman spectroelectrochemistry: Part I. Heterocyclic, aromatic, and aliphatic amines adsorbed on the anodized silver electrode, Journal of Electroanalytical Chemistry and Interfacial Electrochemistry, 1977, 84, p. 1-20.

33. Emory, S. R., Haskins, W. E. and Nie, S., Direct Observation of SizeDependent Optical Enhancement in Single Metal Nanoparticles, Journal of the American Chemical Society, 1998, 120, p. 8009-8010.

34. Haynes, C. L. and Van Duyne, R. P., Plasmon-Sampled Surface-Enhanced Raman Excitation Spectroscopy†, Journal of Physical Chemistry B, 2003, 107, p. 7426-7433.

35. Zeman, E. J. and Schatz, G. C., An accurate electromagnetic theory study of surface enhancement factors for silver, gold, copper, lithium, sodium, aluminum, gallium, indium, zinc, and cadmium, The Journal of Physical Chemistry, 1987, 91, p. 634-643.

36. Krug, J. T., Wang, G. D., Emory, S. R. and Nie, S., Efficient Raman Enhancement and Intermittent Light Emission Observed in Single Gold Nanocrystals, Journal of the American Chemical Society, 1999, 121, p. 92089214.

37. Zihua, Z., Tao, Z. and Zhongfan, L., Raman scattering enhancement contributed from individual gold nanoparticles and interparticle coupling, Nanotechnology, 2004, 15, p. 357.

38. Rodriguez-Fernandez, J., Funston, A. M., Perez-Juste, J., Alvarez-Puebla, R. A., Liz-Marzan, L. M. and Mulvaney, P., The effect of surface roughness on the plasmonic response of individual sub-micron gold spheres, Physical Chemistry Chemical Physics, 2009, 11, p. 5909-5914.

39. Oubre, C. and Nordlander, P., Optical Properties of Metallodielectric Nanostructures Calculated Using the Finite Difference Time Domain Method, Journal of Physical Chemistry B, 2004, 108, p. 17740-17747.

40. Wang, H., Goodrich, G. P., Tam, F., Oubre, C., Nordlander, P. and Halas, N. J., Controlled Texturing Modifies the Surface Topography and Plasmonic Properties of Au Nanoshells, Journal of Physical Chemistry B, 2005, 109, p. 11083-11087.

41. Moskovits, M., Surface-enhanced Raman spectroscopy: a brief retrospective, Journal of Raman Spectroscopy, 2005, 36, p. 485-496. 
42. Svedberg, F., Li, Z., Xu, H. and Käll, M., Creating Hot Nanoparticle Pairs for Surface-Enhanced Raman Spectroscopy through Optical Manipulation, Nano Letters, 2006, 6, p. 2639-2641.

43. Futamata, M., Single molecule sensitivity in SERS: importance of junction of adjacent Ag nanoparticles, Faraday Discussions, 2006, 132, p. 45-61.

44. Blackie, E. J., Ru, E. C. L. and Etchegoin, P. G., Single-Molecule SurfaceEnhanced Raman Spectroscopy of Nonresonant Molecules, Journal of the American Chemical Society, 2009, 131, p. 14466-14472.

45. Lim, D.-K., Jeon, K.-S., Hwang, J.-H., Kim, H., Kwon, S., Suh, Y. D. and Nam, J.-M., Highly uniform and reproducible surface-enhanced Raman scattering from DNA-tailorable nanoparticles with 1-nm interior gap, Nat Nano, 2011, 6, p. $452-460$.

46. Park, W.-H. and Kim, Z. H., Charge Transfer Enhancement in the SERS of a Single Molecule, Nano Letters, 2010, 10, p. 4040-4048. 


\section{Chapter 4-Deep Etched Porous Si Decorated with Au Nanoparticles for SERS}

\subsection{I ntroduction}

Porous solids are of significant importance due to their ability to interact with gases and liquids not only at the surface, but throughout their bulk [1]. As a result, porous nanostructures have exhibited exceptional edges in terms of optical [2], catalytic $[3,4]$, electocatalytic [5], biomedical $[6,7]$, energy storage [8] and electrical $[9,10]$ properties over their bulk solid counterparts. In recent works, porous nanosubstrates, including porous Si [11-13], porous GaN [14], porous $\mathrm{Al}_{2} \mathrm{O}_{3}[15,16]$, porous glass [17], and porous noble metal [18], have been explored for their new application for SERS in attempting to utilize their large surface areas and networked structures. Results from these works strongly support that porous nanosubstrates can serve as high quality SERS substrates with regards to sensitivity, repeatability, and simplicity in fabrication process. 
Among porous nanosubstrates, porous $\mathrm{Si}$ has been the most widely used one for SERS applications as it is a well characterized and readily accessible material. A typical porous Si-based SERS substrate consists of two parts: a porous Si wafer, serving as the base, and a noble metal coating on top of the porous $\mathrm{Si}$, serving as the SERSactive layer. According to previous studies [19-21], porous Si could be readily achieved by electrochemical or electroless etching. As for the SERS-active layer, noble metal deposition is usually conducted in such ways as immersion plating [11], thermal decomposition [12], or physical vapor deposition (PVD) [22]. Herein, we report a simple method of fabricating porous Si-based SERS substrates and present a systematical investigation of SERS dependence on experimental parameters. Although the method was designed on traditional rationale as described by others [11-13], our study explored new approaches with unique findings compared to previous works. Firstly, rather than shallow etching, we extended the etching process to relatively long periods and identified the significance of deep etching on SERS performance. Secondly, besides the analysis of SERS dependence on individual experimental parameter, namely Au coating thickness and etching time, we examined the combinational effect of these two parameters on SERS in order to optimize the SERS substrates. In the end, we, for the first time, employed Au nanoisland 
arrays instead of plain PVD Au as catalysts for metal-assisted wet etching in hopes of obtaining better SERS robustness, repeatability, and enhancement.

\subsection{Experimental section}

\subsubsection{Fabrication of SERS substrates}

P-type Si wafer with (100) orientation was rinsed with acetone, methanol, and de-ionized (DI) water in sequence. The native oxide was not removed since it was not considered critical for the etching process [23]. An ultra thin film of Au ( $5 \mathrm{~nm}$ nominal thickness) was sputtered onto Si wafer by an Emitech K650X sputter coater. In this study, the nominal film thickness was measured by a built-in quartz crystal balance. Then the Au-deposited substrate was annealed in a tube furnace at $200{ }^{\circ} \mathrm{C}$ for $2 \mathrm{~h}$, accompanied by 200 standard cubic centimeters per minute $(\mathrm{sccm})$ gas mixture of $\operatorname{Ar}(95 \%)$ and $\mathrm{H}_{2}(5 \%)$. Hereafter, wet etching was performed at room temperature without light by immersing the annealed substrates into a freshly prepared aqueous solution which was composed of $\mathrm{HF}(49 \%), \mathrm{H}_{2} \mathrm{O}_{2}(30 \%)$, and DI water in volumetric ratio of $2: 1: 7$. The etching process was set up for various periods of time, namely $0.5,1,2,4,6,8,10,15,20,30$, and $40 \mathrm{~min}$. In this work, $0.5-2 \mathrm{~min}, 4-8 \mathrm{~min}$, and $10-40 \mathrm{~min}$ are referred as shallow, medium, and deep etching, respectively. In the 
end, a layer of Au thin film $(30,50,70,90,120,150,200$, and 300 $\mathrm{nm}$ in nominal thicknesses) was deposited over the as-etched substrates by the abovementioned Emitech sputtering machine to finish the fabrication of SERS substrates. In addition, $50 \mathrm{~nm}$ Au film sputtered onto bare Si wafer (without $5 \mathrm{~nm}$ Au pre-coating, annealing, or etching) was prepared as the reference for evaluating SERS enhancement factor. The morphological characterization of SERS substrates was taken with a field-emission scanning electron microscope (SEM, Hitachi S-4700). The surface roughness analysis was carried out with a tapping mode atomic force microscope (AFM, Agilent 5500) and an image processing package (Gwyddion). Specifically, root mean square (RMS) surface roughness was collected from SERS substrates based on 0.5 ( 500 by $500 \mathrm{~nm}$ scan size), 10, ( 5 by $5 \mu \mathrm{m}$ scan size) and $30 \mathrm{~min}$ ( 5 by $5 \mu \mathrm{m}$ scan size) porous $\mathrm{Si}$.

\subsubsection{SERS measurements}

The spectrometer employed in Raman analysis was a B\&W Tek iRaman portable system equipped with a $785 \mathrm{~nm}$ near-infrared diode laser. SERS spectra were collected with $40 \mathrm{~mW}$ output power and $10 \mathrm{~s}$ acquisitions. In this study, 1,2-benzenedithiol (BDT, Sigma-Aldrich) was used as the analyte without further purification. BDT analyte solutions with various concentrations were prepared in 200-proof 
ethanol. Prior to measurements, SERS substrates were incubated in BDT analyte solutions for $1 \mathrm{~h}$ and then dried in air.

\subsubsection{Effects of predefined Au nanoisland arrays by annealing}

In our study, Au nanoisland arrays were created by annealing plain sputtered Au prior to etching. In order to evaluate the effects of predefined Au nanoisland arrays, alternatively, annealing, on SERS performance, a comparison between annealed (Au nanoisland arrays) and un-annealed (plain sputtered $\mathrm{Au}$ ) substrates was carried out; that is, $5 \mathrm{~nm}$ (nominal thickness) Au was deposited on two pieces of Si wafers, then one of them was annealed as described in section 4.2.1 while the other one was not. Hereafter, both substrates were etched, sputtered with $\mathrm{Au}$ (30-300 $\mathrm{nm}$ in nominal thickness), and tested for SERS measurements. For simplicity, only one etching condition (10 min) was selected for this comparison. In addition, this experiment was conducted three times independently in order to investigate the repeatability.

\subsection{Results and discussion}

\subsubsection{Metal-assisted wet etching of $\mathrm{Si}$}

During a typical metal-assisted wet etching process, the chemical reaction preferentially takes place at the interface between 
noble metal and $\mathrm{Si}$. As a result, the $\mathrm{Si}$ beneath noble metal is etched much faster than the Si without metal coverage. The etching in our work was designed on such mechanism with minor modification. Specifically, instead of using plain sputtered Au as the catalyst, we annealed sputtered Au to create nanoisland arrays prior to etching. The primary purpose of this annealing was to stabilize Au on Si for the subsequent etching process and achieve better SERS performance. Detailed results and discussion supporting this objective will be presented in section 4.3.5.

SEM image in Fig. 4.1(a) shows Au nanoisland arrays of $\sim 15$ $\mathrm{nm}$ in average island size and $\sim 15 \mathrm{~nm}$ in average inter-island spacing uniformly distributing over the Si wafer after the annealing of $5 \mathrm{~nm}$ ultra thin Au film. Once this Au-coated Si was in contact with etchant, the pores were expected to form at the sites of Au nanoislands. In this study, time-dependent samples were utilized to monitor the morphological evolution of pore structure. Figure 4.1(b) shows that the pores were quickly formed after 0.5 min etching. As the reaction continued, some pores started to merge due to lateral etching, as evident by the image of 5 min etched porous $\mathrm{Si}$ in Fig. 4.1(c). Moreover, the cross sectional view of 5 min etched porous Si (inset of Fig. 4.1(c)) reveals Au nanoislands sitting at the bottom of pores. This observation implies that $\mathrm{Au}$ nanoislands sank into $\mathrm{Si}$, which 
confirms their role of catalyzing in the etching process. When the etching was extended to $10 \mathrm{~min}$, the porous Si transitioned into a sponge-like structure as shown in Fig. 4.1(d). In addition, it is worthwhile mentioning that samples with further longer etching time showed no significant morphological variation compared to $10 \mathrm{~min}$ etched porous Si.

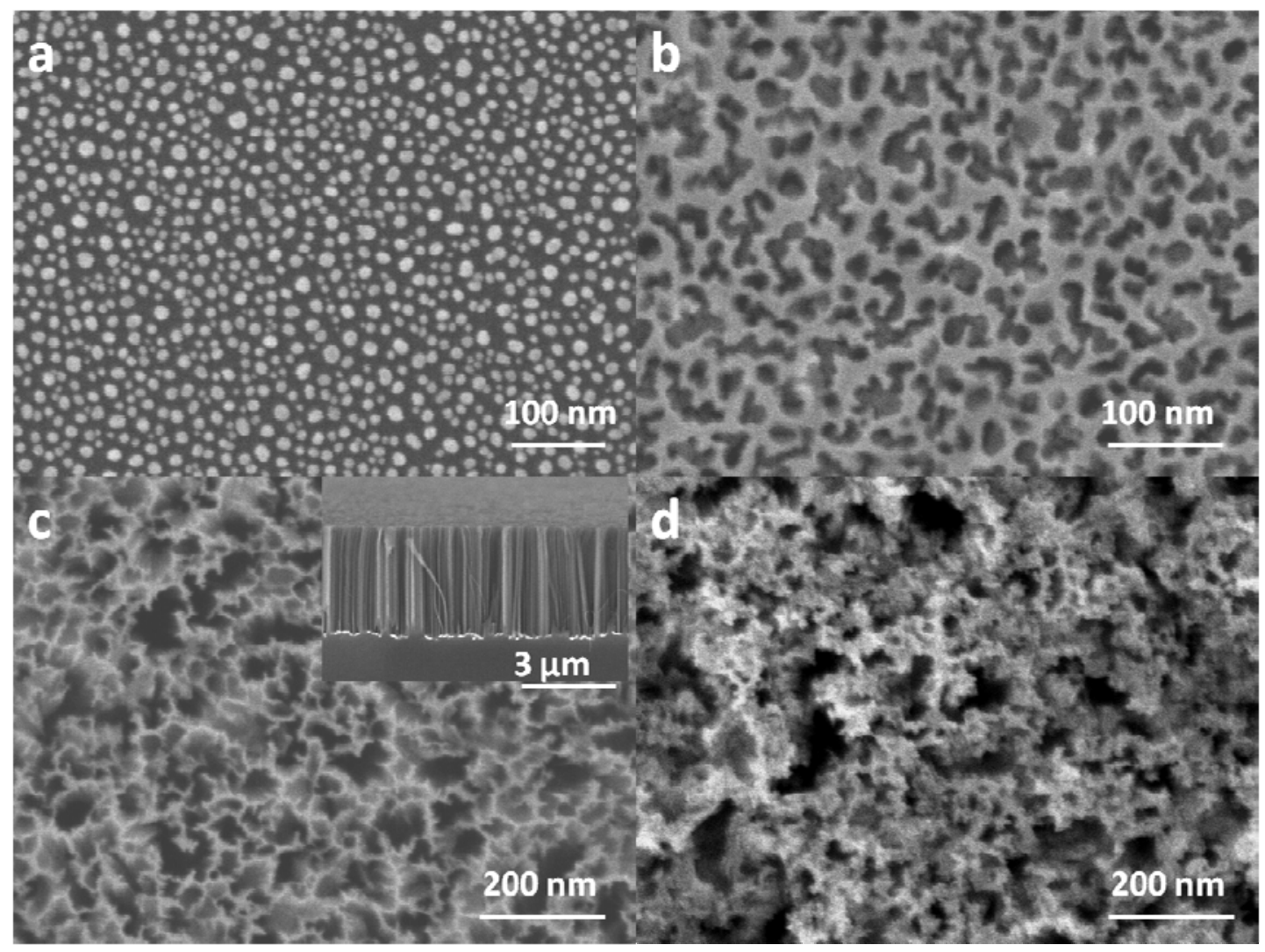

Figure 4.1. Top view SEM images of Au nanoislands arrays (a) and porous $\mathrm{Si}$ fabricated from (a) with $0.5 \mathrm{~min}$ (b), $5 \mathrm{~min}$ (c) and $10 \mathrm{~min}$ (d) etching periods. Cross sectional SEM image of 5 min etched porous Si (inset of $c$ ).

\subsubsection{Morphology of SERS substrates}


In this work, porous $\mathrm{Si}$ with a host of etching periods $(0.5-40$ min) were prepared for study. Apparently, morphology of these porous Si varied greatly both in-plane and vertical. Hence, in order to obtain the complete understanding of SERS dependence, we accordingly investigated the Au coating with thickness ranging from very thin (30 $\mathrm{nm}$ ) to very thick $(300 \mathrm{~nm})$. Selective SEM images of these SERS substrates are exhibited in Fig. 4.2.

Figure 4.2(a)-(d) illustrate the morphological evolution of SERS substrates based on 0.5 min (shallow) etched porous Si. These images represent SERS substrates with 30,90, 150, and $300 \mathrm{~nm} \mathrm{Au}$ coatings, respectively. With $30 \mathrm{~nm}$ Au coating (Fig. 4.2(a)), the pores were still observable and the substrate in general appeared similar to the nanoforest structure reported by M.L. Seol et al. [13] as they had experimental conditions (10-70 s etching and $50 \mathrm{~nm}$ Au coating) close to ours. We purposely included these parameters in our data set for the comparison with medium and deep etching. When the Au coating accumulated to $90 \mathrm{~nm}$, most of the pores were closed up by the sputtered Au as shown in Fig. 4.2(b). Figure 4.2(c) depicts the continuing enclosure process as the Au coating built up to $150 \mathrm{~nm}$. Finally, with the thickest Au coating of $300 \mathrm{~nm}$ (Fig. 4.2(d)), the morphology of the SERS substrate was virtually same as that of the 


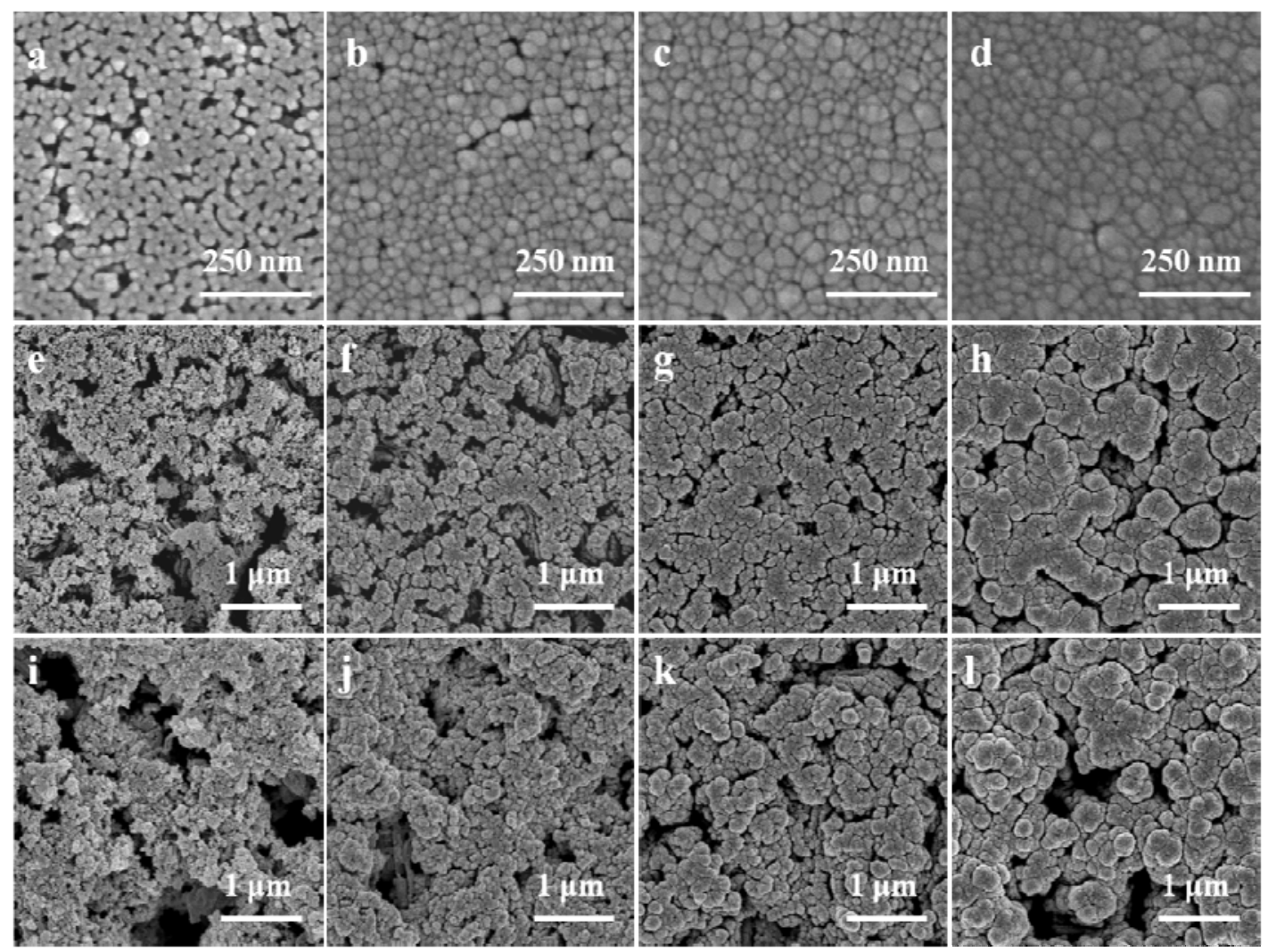

Figure 4.2. Top row: top view SEM images of $30 \mathrm{~nm}$ (a), $90 \mathrm{~nm}$ (b), $150 \mathrm{~nm}$ (d), and $300 \mathrm{~nm}$ (d) Au coatings on 0.5 min etched porous Si, respectively. Middle row: top view SEM images of $30 \mathrm{~nm}(\mathrm{e}), 90 \mathrm{~nm}(\mathrm{f}), 150 \mathrm{~nm}(\mathrm{~g})$, and $300 \mathrm{~nm}(\mathrm{~h})$ Au coatings on 10 min etched porous $\mathrm{Si}$, respectively. Bottom row: top view SEM images of $30 \mathrm{~nm}$ (i), $90 \mathrm{~nm}(\mathrm{j}), 150 \mathrm{~nm}(\mathrm{k})$, and $300 \mathrm{~nm}$ (I) Au coatings on $30 \mathrm{~min}$ etched porous $\mathrm{Si}$, respectively.

sputtered Au directly on bare Si wafer, which would lead to inferior SERS performance.

With regards to SERS substrates based on 10 min (deep) etched porous Si, Fig. 4.2(e)-(h) show their morphology with 30, 90, 150, and $300 \mathrm{~nm}$ Au coatings, respectively. As mentioned in section 4.3.1, sponge-like porous Si was formed under this etching condition. In fact, even with $30 \mathrm{~nm}$ Au coating (Fig. 4.2(e)), the SERS substrate 
remained sponge-like as if without Au. When the Au coating reached $90 \mathrm{~nm}$ (Fig. 4.2(f)), the branches of porous Si were covered and the trunk of porous Si presented itself with Au nanoparticles as decoration, indicating the completion of Au coverage on porous Si surface. Figure 4.2(g) shows the pores were filled to a certain degree as the Au coating went up to $150 \mathrm{~nm}$. With $300 \mathrm{~nm}$ Au coating, a relatively smooth surface was formed because of pores filling, shown in Fig. 4.2(h). Furthermore, Fig. 4.2(i)-(I) list the SEM images of 30, 90, 150, and $300 \mathrm{~nm}$ Au coatings on 30 min etched porous Si, respectively. These images suggest that SERS substrates based on 30 min etched porous Si were very similar to SERS substrates based on $10 \mathrm{~min}$ etched porous $\mathrm{Si}$, despite the morphological evolution of 30 min etched SERS substrates lagged a slight extent with increasing Au coating thickness.

\subsubsection{SERS dependence on Au coating thickness and etching time}

To study the dependence of SERS on Au coating thickness and etching time, we conducted SERS measurements on various SERS substrates with all other experimental parameters being equal, including laser power, acquisition time, and analyte concentration. Please note, under such circumstances, the Raman intensity of each 
spectrum was a direct indicator of the SERS substrate's enhancement factor (EF), according to the definition of EF [24]. Therefore, in this study, SERS enhancement was evaluated by analyzing the Raman intensity at $v=1030 \mathrm{~cm}^{-1}$ of $10^{-4} \mathrm{M}$ BDT instead of the completely converted EF.

Figure 4.3 exhibits the relationship between Raman intensity and $\mathrm{Au}$ coating thickness for a series of SERS substrates with varying etching periods. Curves of shallow (0.5-2 min) etched SERS substrates indicate that the Raman intensity initially increased with increasing $\mathrm{Au}$ coating thickness till it reached maximum. Then, as Au coating passed beyond the optimal thickness, the Raman intensity started to fall and even eventually regressed to zero for cases of 0.5 and 1 min etched SERS substrates (data showing no Raman signal was not plotted in Fig. 3). Similarly, curves of medium (4-8 $\mathrm{min}$ ) and deep (10-40 min) etched SERS substrates show that the Raman intensity followed the same pattern of rising up to maximum and then declining with increasing Au coating thickness. In order to explain such relationship, electromagnetic enhancement (EM) mechanism of SERS was firstly examined. According to EM mechanism [25-30], surface roughness at nanometer scale induces localized surface plasmon resonance (LSPR) that significantly amplifies scattered electromagnetic field and Raman intensity. In this study, it is shown in Fig. $\mathbf{4 . 2}$ along horizontal 
direction that the surface roughness seemed to gradually diminish with increasing Au coating. To confirm it, AFM images were taken and root mean square (RMS) surface roughness was measured on all SERS substrates shown in Fig. 4.2. Figure 4.4 clearly indicates that surface roughness decreased monotonically with thicker $\mathrm{Au}$ coating for all three representative etching conditions. We speculate this loss of surface roughness accounted for the decreasing part of each curve. On the other hand, the increasing part might be attributed to the degree of $\mathrm{Au}$ coverage on porous $\mathrm{Si}$ surface. Specifically, there was no complete Au coverage when the Au coating was too thin, and, in turn,

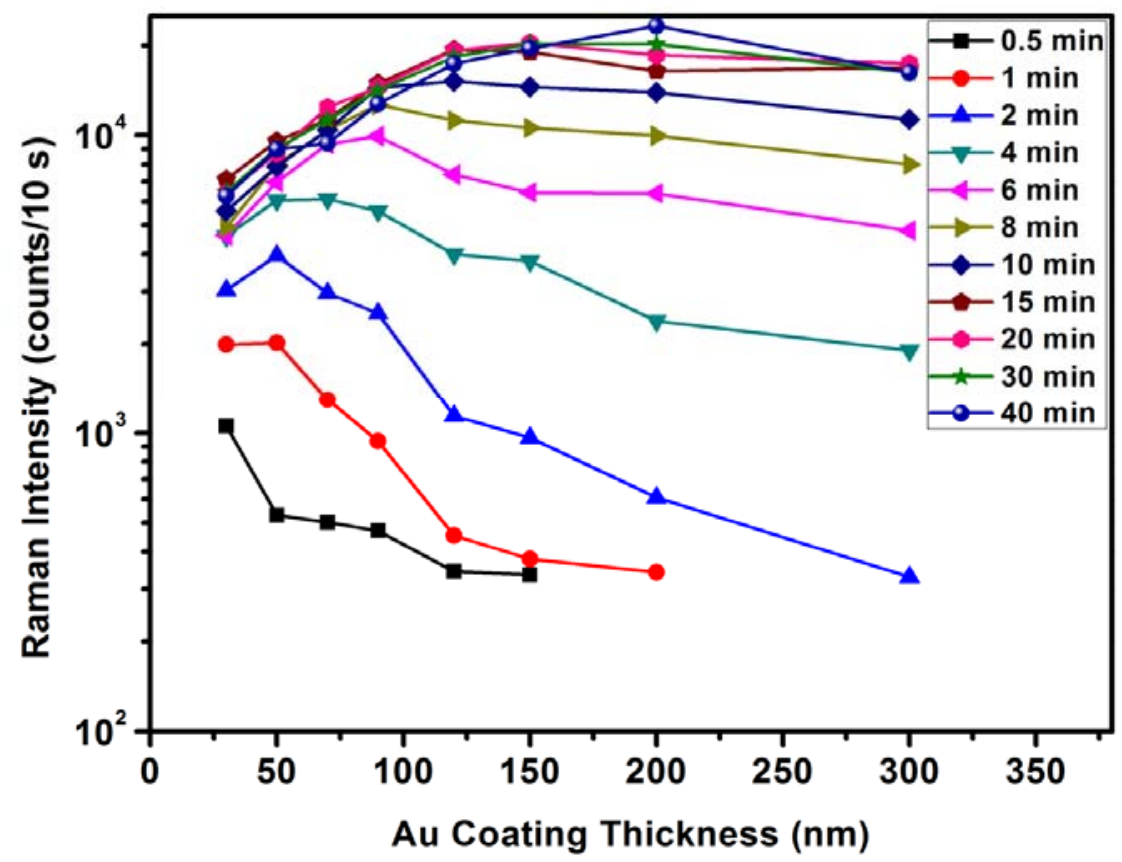

Figure 4.3. Au coating thickness dependence of Raman intensity $\left(v=1030 \mathrm{~cm}^{-1}\right.$ of $10^{-4} \mathrm{M}$ BDT). 
the Raman intensity was expected to be low. As the Au coverage neared toward completion, the Raman intensity climbed correspondingly. At the moment of achieving complete coverage, the Raman intensity reached maximum with the minimized trade-off of surface roughness.

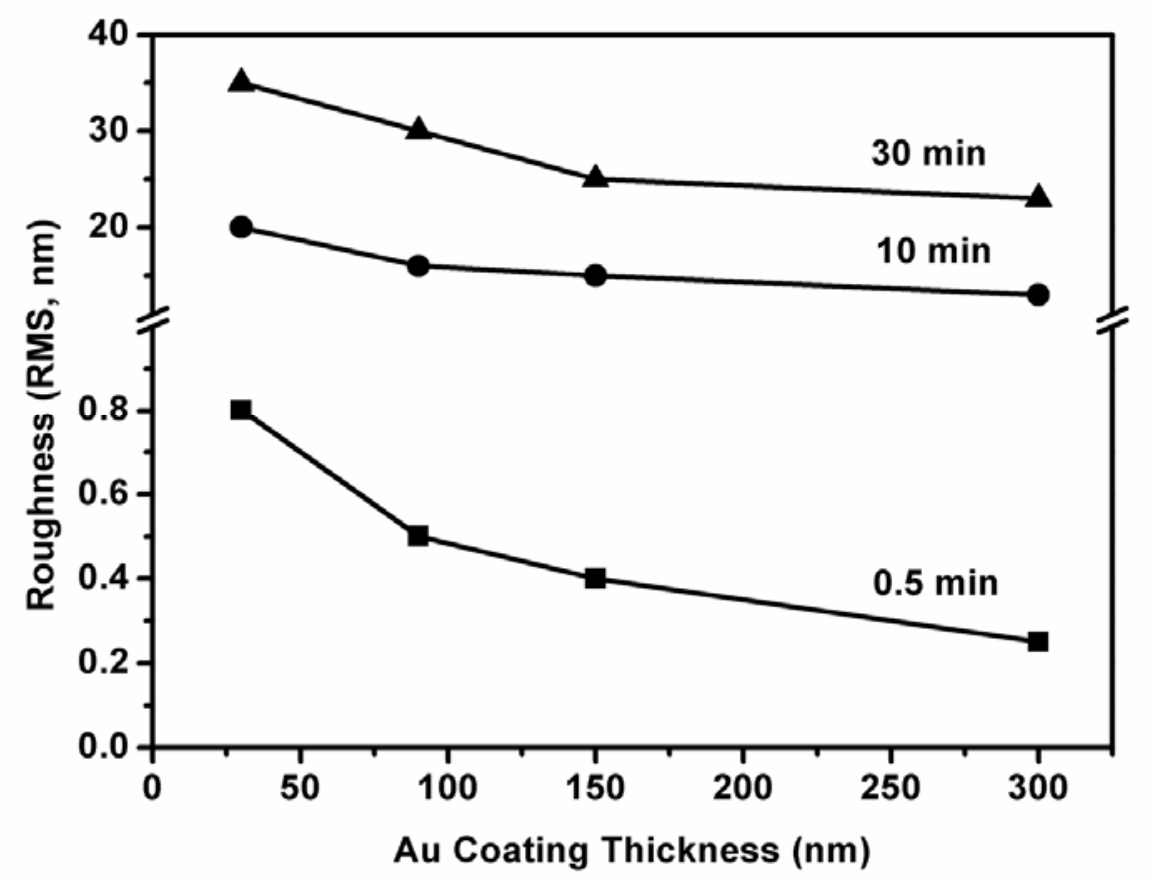

Figure 4.4. Relationship between root mean square (RMS) surface roughness and Au coating thickness for three representative etching periods $(0.5 \mathrm{~min}, 10 \mathrm{~min}$, and $30 \mathrm{~min}$ ).

Furthermore, Fig. 4.3 shows that the optimal Au coating thickness shifted to right with the extended etching time. For example, the optimal Au coating thicknesses were $\sim 50, \sim 100$, and $\sim 150 \mathrm{~nm}$ for 1,8 , and 20 min etched SERS substrates, respectively. In fact, the 
right shift could also be explained by the degree of Au coverage on porous Si surface. As shown in Fig. 4.2 along vertical direction, the pores merged and formed structures in larger dimensions with the extended etching time. As a consequence, the amount of Au needed for complete coverage on porous Si surface increased correspondingly, resulting in a right shift of the optimal $A u$ coating thickness. In addition, Fig. 4.3 indicates that the Raman intensity was overall boosted by extending the etching time, hinted by the stacking order of curves. For instance, Raman intensity of 30 min etched SERS substrate was about an order of magnitude higher than that of 1 min etched SERS substrate, regardless of Au coating thickness.

In order to better present the underlying relationship, we plotted Fig. 4.5 with same data as in Fig. 4.3, but set etching time as $x$-axis instead. As shown in Fig. 4.5, each curve suggests that the Raman intensity sharply increased by an order of magnitude when the etching was prolonged from 0.5 to $10 \mathrm{~min}$. To unveil the cause to this enormous gain, we compared panel (a) with (e), (b) with (f), (c) with (g), and (d) with (h) in Fig. 4.2 and found that the surface morphology of SERS substrates changed dramatically from 0.5 to 10 min etching. Surface roughness analysis in Fig. $\mathbf{4 . 4}$ confirms that 10 min etching increased surface roughness by about two orders of magnitude over $0.5 \mathrm{~min}$ etching. Apparently, this huge creation of 


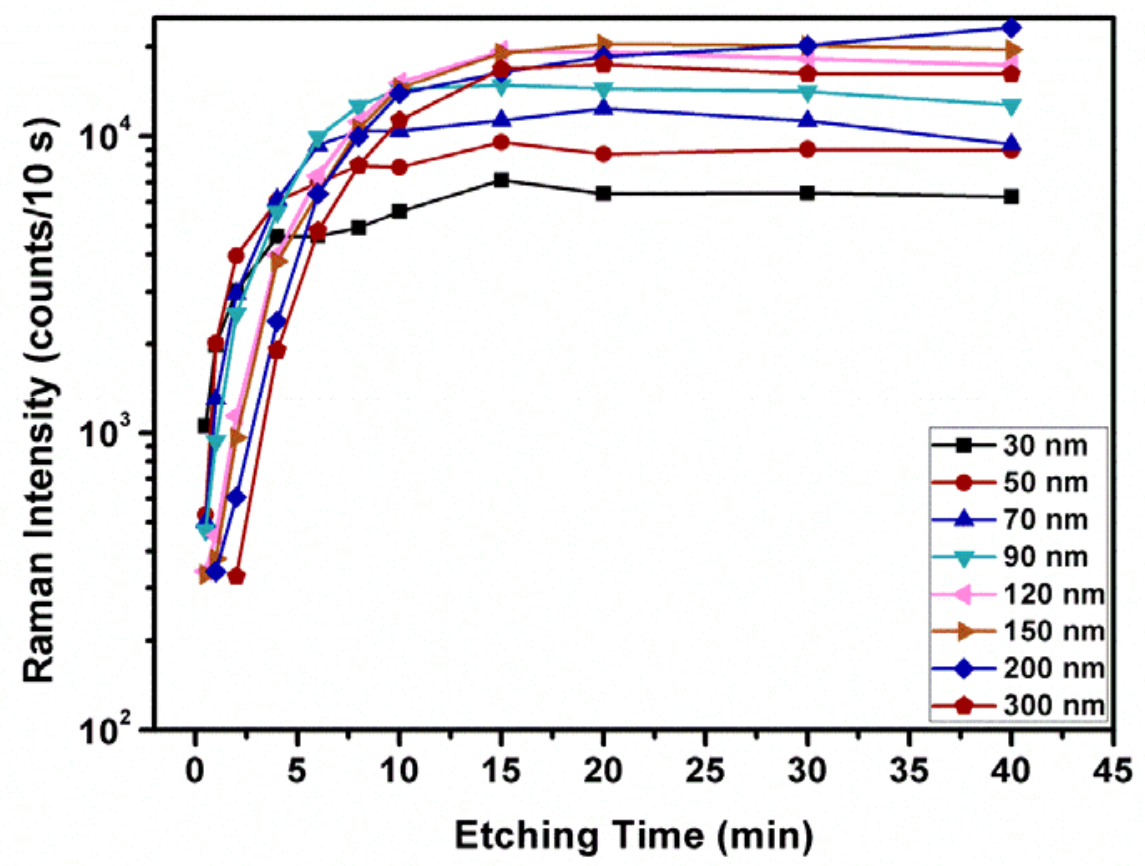

Figure 4.5. Etching time dependence of Raman intensity $\left(v=1030 \mathrm{~cm}^{-1}\right.$ of $10^{-4} \mathrm{M}$ BDT).

surface roughness contributed to the observed jump in Raman intensity. Still shown in Fig. 4.5, the Raman intensity of each curve remained nearly constant after etching time passed by $10 \mathrm{~min}$. Again, we sought explanation for this relationship from the perspective of surface morphological variation. Comparisons of panel (e) with (i), (f) with (j), (g) with (k), and (h) with (I) in Fig. 4.2 suggest that the surface morphology of SERS substrates changed limitedly as the etching was prolonged from 10 to $30 \mathrm{~min}$. As confirmed in Fig. 4.4, surface roughness of 10 and 30 min etched SERS substrates was in 
the same order of magnitude, which accounted for the nearly constant in Raman intensity. Lastly, unlike curves in Fig. 4.3, curves as a whole in Fig. 4.5 stay more compact, suggesting that Au coating thickness played less important role than etching time did in SERS performance. Specifically, with fixed etching time, the difference in Raman intensity between $30 \mathrm{~nm}$ and $300 \mathrm{~nm}$ Au coated SERS substrates was no more than three times, as evidenced in Fig. 4.5.

In addition to the individual analysis on Au coating thickness and etching time, the combinational effect of these two parameters was also examined in this study. A contour map representing the distribution of Raman intensity with varying Au coating thickness and etching time is shown in Fig. 4.6(a). The map clearly suggests that the deepest etched porous Si $(40 \mathrm{~min})$ coupled with $200 \mathrm{~nm} \mathrm{Au}$ coating yielded the highest enhancement, with its representative SEM image shown in Fig. 4.6(b). Moreover, the map hints an increasing tendency of Raman intensity along the diagonal pointing to the bottom-right direction. In light of this trend, we predict that further deeper etching coupled with further thicker Au coating is potentially capable of raising the Raman intensity to a further higher level. 


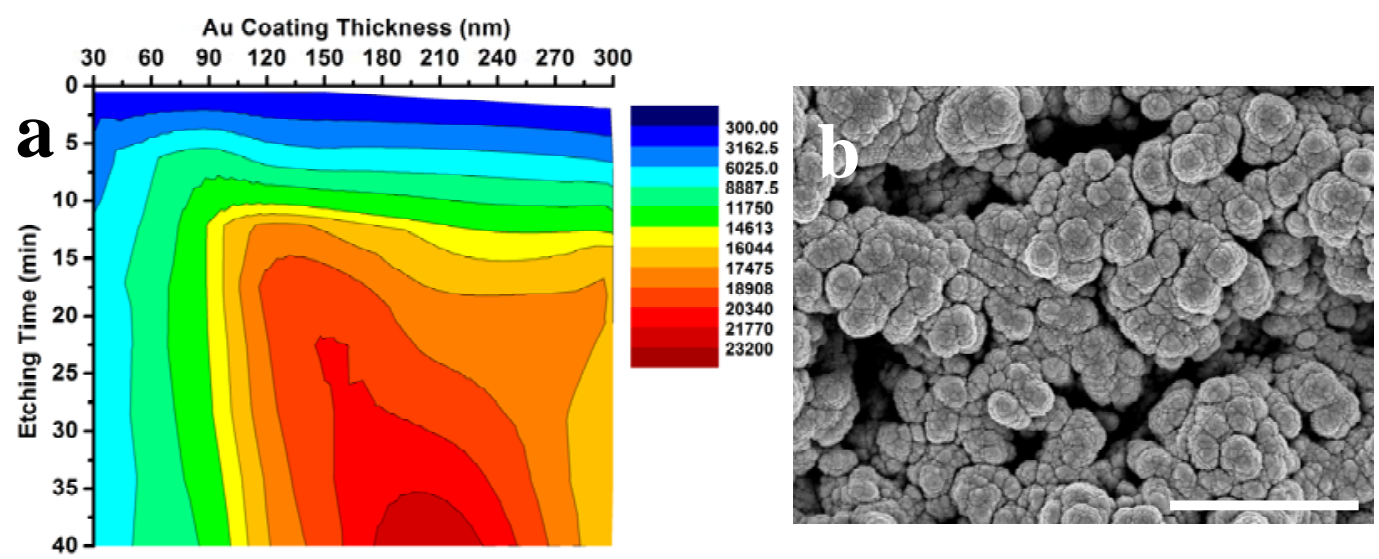

Figure 4.6. (a) Contour map of Raman intensity at $v=1030 \mathrm{~cm}^{-1}$ of $10^{-4} \mathrm{M}$ BDT solution with respect to two parameters: Au coating thickness and etching time. (b) SEM image of SERS substrate with optimized parameters: 40 min etched porous Si and $200 \mathrm{~nm}$ Au coating. Scale bar represents $1 \mu \mathrm{m}$.

\subsubsection{Evaluations of enhancement factor and uniformity}

Enhancement factor is one of the most important parameters for characterizing SERS substrates and has various forms of expressions [24]. In this study, we chose analytical enhancement factor (AEF) to quantify our SERS substrate. AEF is defined as following,

$$
\mathrm{AEF}=\left(\mathrm{C}_{\mathrm{R}} / \mathrm{C}_{\mathrm{SERS}}\right) *\left(\mathrm{I}_{\mathrm{SERS}} / \mathrm{I}_{\mathrm{R}}\right)
$$

where $C_{S E R S}$ and $C_{R}$ are concentrations of analyte solutions for SERS and non-SERS measurements, respectively. $I_{S E R S}$ and $I_{R}$ are Raman intensities of the same peak for SERS and non-SERS measurements performed under exactly same conditions. Here, the SERS substrate we used for evaluation was a 20 min etched porous Si with $150 \mathrm{~nm} \mathrm{Au}$ 
coating and the SERS analyte was $10^{-5} \mathrm{M}$ BDT. For reference, we measured non-SERS signal of $10^{-1} \mathrm{M}$ BDT on $50 \mathrm{~nm}$ Au coated bare Si wafer. The Raman intensities at $\mathrm{v}=1030 \mathrm{~cm}^{-1}$ for two measurements were recorded for AEF calculation. Figure 4.7 shows that the SERS signal was almost 20 times as high as that of non-SERS. According to AEF equation, an enhancement factor of $2 \times 10^{5}$ was obtained for this SERS substrate over bare Au film, indicative of high sensitivity considering the reference material used here was $\mathrm{Au}$.

In addition, we randomly collected BDT Raman spectra on this

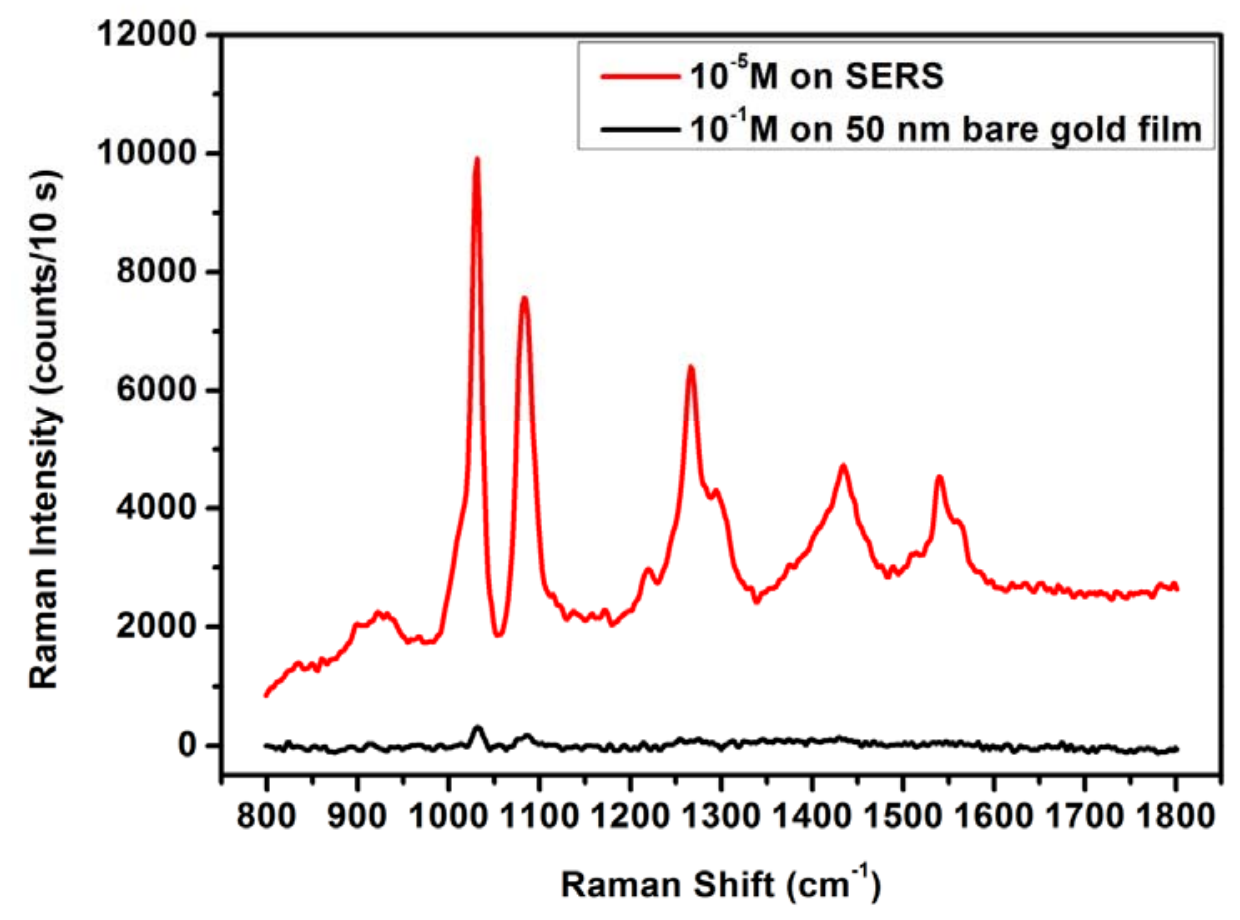

Figure 4.7. SERS spectrum of $10^{-5} \mathrm{M}$ BDT acquired on 20 min etched porous $\mathrm{Si}$ with $150 \mathrm{~nm}$ Au coating (red). Raman spectrum of $10^{-1} \mathrm{M}$ BDT acquired on $50 \mathrm{~nm}$ Au film on un-etched Si (black). 
SERS substrate $(1 \mathrm{~cm}$ by $1 \mathrm{~cm}$ ) as many as 20 times to characterize its uniformity. Figure $\mathbf{4 . 8}$ shows good resemblance among 20 acquisitions, suggesting the substrate possessed high repeatability that is usually considered as a valuable attribute for practical applications. In addition, a quantitative study was carried out by analyzing the peak $\left(v=1030 \mathrm{~cm}^{-1}\right)$ intensities of these 20 acquisitions. The relative standard deviation (RSD) is calculated to be $4.4 \%$ on this $1 \mathrm{~cm}$ by $1 \mathrm{~cm}$ SERS substrate, consistent with the resemblance presented in Fig. 4.8. Although the RSD appears promising, it should be admitted that the scale of this SERS substrate is too small to be applied for production. More work is needed to explore if the repeatability can hold up for scaled up samples. 


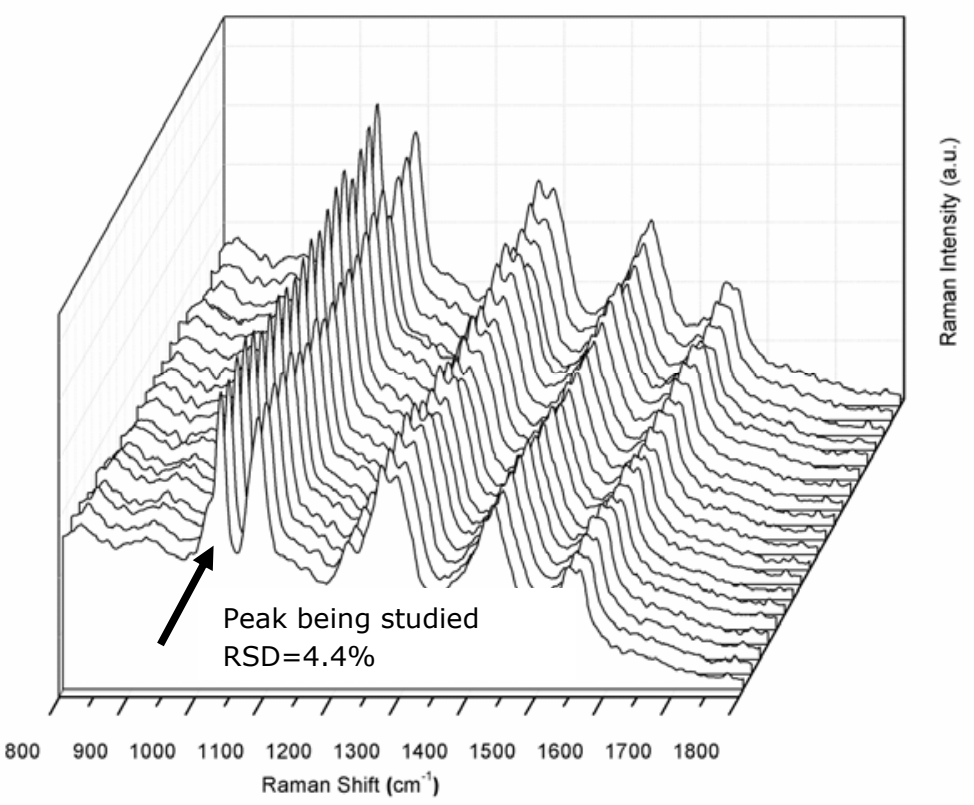

Figure 4.8. Raw SERS spectra of $10^{-5} \mathrm{M}$ BDT collected at 20 random spots on the SERS substrate $(1 \mathrm{~cm}$ by $1 \mathrm{~cm})$ of Fig. 4.7.

\subsubsection{Effects of predefined Au nanoisland arrays by annealing}

While wet etching of $\mathrm{Si}$ assisted by physical vapor deposited metals has been reported elsewhere [31, 32], the present work introduced one more step in between metal deposition and Si etching: annealing. The objective for this extra step was to stabilize Au on $\mathrm{Si}$ as it is commonly known that Au film does not adhere well to Si wafer due to the inhomogeneous stress distribution across the Au-Si interface [33]. The adhesion issue is particularly active when immersing Au-coated $\mathrm{Si}$ into aqueous medium as the surface tension may break Au film badly. To solve this problem, annealing is a viable 
option as it facilitates the migration of $\mathrm{Au}$ atoms at the interfaces to more relaxed regions, leaving a more stable Au-Si interface. Moreover, annealing gives rise to the formation of quasi-periodic Au nanoisland arrays, which may contribute to better SERS repeatability and enhancement.

In this study, we examined the effects of predefined $\mathrm{Au}$ nanoisland arrays, alternatively, annealing, by comparing the performance of SERS substrates etched from Au nanoisland arrays (annealed) and plain sputtered Au (un-annealed). The comparison was evaluated in aspects of SERS (i) robustness, (ii) uniformity within a batch, and (iii) enhancement. Firstly, during all three batches of independent experiments, the annealed substrates exhibited robustness when they were immersed into etching solutions, while small pieces of $\mathrm{Au}$ film indeed encountered peeling off for the unannealed substrates. It should be pointed out that no Raman spectrum was taken at those broken spots for the following test. Secondly, the relationship between Raman intensity and Au coating thickness for a 10 min etched SERS substrate (Fig. 4.9(a)) indicates that annealed substrates (red curves) were superior to un-annealed substrates (blue curves) in terms of repeatability among batches, as evident by the better repeated red curves. Figure 4.9(b) quantitatively confirms such a claim by presenting the relative standard deviation (RSD) of 
Raman intensity among three batches, where RSDs in the range of $1-5 \%$ and $2-25 \%$ were concluded for annealed (red columns) and unannealed (blue columns) substrates, respectively. Lastly, given the same Au coating thickness, Fig. 4.9(a) suggests that the Raman intensities of annealed substrates were slightly but unanimously higher than those of un-annealed substrates. In summary, our results demonstrated that using Au nanoisland arrays as etching catalyst led to better SERS performance in terms of robustness, batch to batch
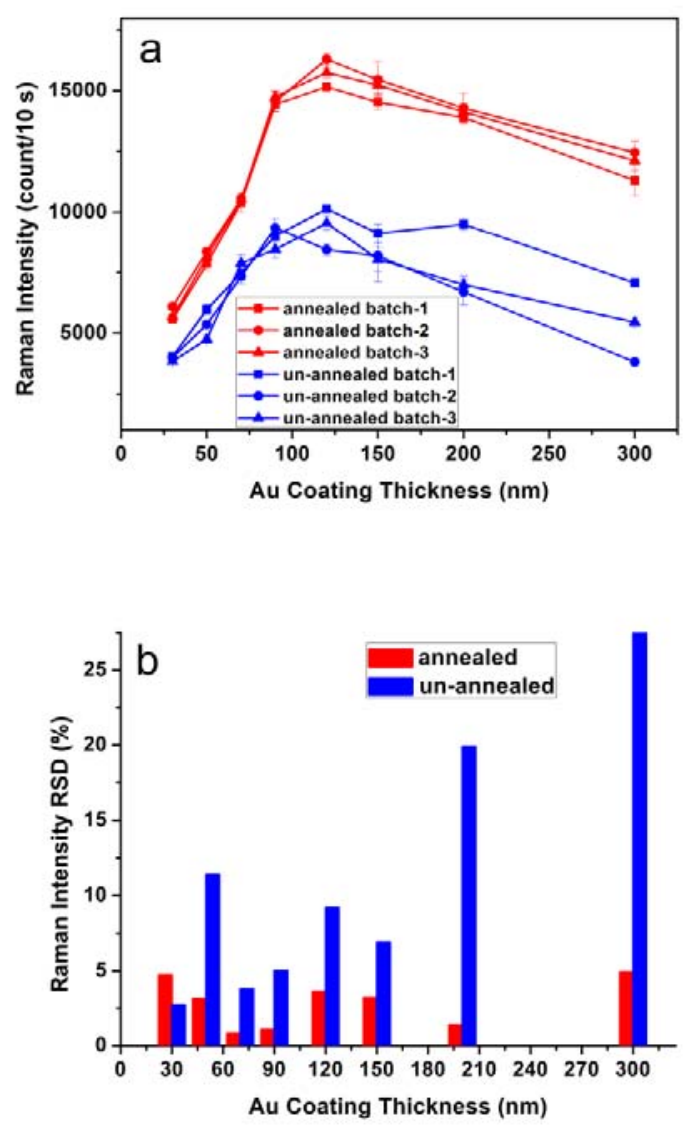

Figure 4.9. (a) Relationship between Raman intensity $\left(v=1030 \mathrm{~cm}^{-1}\right.$ of $10^{-}$ ${ }^{4} \mathrm{M} \mathrm{BDT}$ ) and Au coating thickness for SERS substrates based on $10 \mathrm{~min}$ etched porous Si. Red and blue curves represent SERS substrates etched from annealed and un-annealed substrates, respectively. (b) RSD of Raman intensity among three batches of experiments shown in (a). Red and blue bars represent SERS substrates etched from annealed and un-annealed substrates, respectively. 
repeatability, and enhancement. Further study is underway to establish a more in-depth understanding of the influences of predefined Au nanoisland arrays on SERS, as well as the mechanisms behind the observed difference in SERS enhancement.

\subsection{Conclusion}

Au nanoparticles decorated porous Si for SERS applications was prepared via metal-assisted wet etching and sputtering processes. Our work placed an emphasis on the influence of deep etching and concluded that deep etching increased SERS enhancement factor by an order of magnitude over shallow etching. In addition, the optimized experimental parameters for SERS substrates were obtained by investigating the SERS dependence on $\mathrm{Au}$ coating thickness and etching time. Moreover, the enhancement factor and uniformity within a batch of our SERS substrate were demonstrated to be very promising. Lastly, the effects of predefined Au nanoisland arrays (annealing) were experimentally proven beneficial for SERS performance, in terms of robustness, batch to batch repeatability, and enhancement.

\subsection{Reference}


1. Davis, M. E., Ordered porous materials for emerging applications, Nature 2002, 417, p. 813-821.

2. Biener, J., Nyce, G. W., Hodge, A. M., Biener, M. M., Hamza, A. V. and Maier, S. A., Nanoporous Plasmonic Metamaterials, Advanced Materials, 2008, 20, p. 1211-1217.

3. Wittstock, A., Zielasek, V., Biener, J., Friend, C. M. and Bäumer, M., Nanoporous Gold Catalysts for Selective Gas-Phase Oxidative Coupling of Methanol at Low Temperature, Science, 2010, 327, p. 319-322.

4. Chen, L., Yang, Y. and Jiang, D., CMPs as Scaffolds for Constructing Porous Catalytic Frameworks: A Built-in Heterogeneous Catalyst with High Activity and Selectivity Based on Nanoporous Metalloporphyrin Polymers, Journal of the American Chemical Society, 2010, 132, p. 9138-9143.

5. Zeis, R., Mathur, A., Fritz, G., Lee, J. and Erlebacher, J., Platinum-plated nanoporous gold: An efficient, low Pt loading electrocatalyst for PEM fuel cells, Journal of Power Sources, 2007, 165, p. 65-72.

6. Yoshikawa, H. and Myoui, A., Bone tissue engineering with porous hydroxyapatite ceramics, Journal of Artificial Organs, 2005, 8, p. 131-136.

7. Chang, B.-S., Lee, gt, inits, C.K, fnm, Choon, K., Hong, K.-S., Youn, H.-J., Ryu, H.-S., Chung, S.-S. and Park, K.-W., Osteoconduction at porous hydroxyapatite with various pore configurations, Biomaterials, 2000, 21, p. 1291-1298.

8. Morris, R. E. and Wheatley, P. S., Gas Storage in Nanoporous Materials, Angewandte Chemie International Edition, 2008, 47, p. 4966-4981.

9. Danishevskii, A. M., Popov, V. V., Kyutt, R. N. and Gordeev, S. K., Electrical and galvanomagnetic properties of nanoporous carbon samples impregnated with bromine, Physics of the Solid State, 2013, 55, p. 1487-1493.

10. Wahl, P., Traussnig, T., Landgraf, S., Jin, H.-J., Weissmuller, J. and Wurschum, R., Adsorption-driven tuning of the electrical resistance of nanoporous gold, Journal of Applied Physics, 2010, 108, p. 073706-6.

11. Panarin, A. Y., Terekhov, S. N., Kholostov, K. I. and Bondarenko, V. P., SERSactive substrates based on n-type porous silicon, Applied Surface Science, 2010, 256, p. 6969-6976. 
12. Chan, S., Kwon, S., Koo, T. W., Lee, L. P. and Berlin, A. A., Surface-Enhanced Raman Scattering of Small Molecules from Silver-Coated Silicon Nanopores, Advanced Materials, 2003, 15, p. 1595-1598.

13. Myeong-Lok, S., Sung-Jin, C., David, J. B., Tae Jung, P., Jae-Hyuk, A., Sang Yup, L. and Yang-Kyu, C., A nanoforest structure for practical surfaceenhanced Raman scattering substrates, Nanotechnology, 2012, 23, p. 095301.

14. Williamson, T. L., Guo, X., Zukoski, A., Sood, A., Díaz, D. J. and Bohn, P. W., Porous GaN as a Template to Produce Surface-Enhanced Raman ScatteringActive Surfaces, The Journal of Physical Chemistry B, 2005, 109, p. 2018620191.

15. Du, Y., Shi, L., He, T., Sun, X. and Mo, Y., SERS enhancement dependence on the diameter and aspect ratio of silver-nanowire array fabricated by anodic aluminium oxide template, Applied Surface Science, 2008, 255, p. 1901-1905.

16. Choi, D., Choi, Y., Hong, S., Kang, T. and Lee, L. P., Self-Organized Hexagonal-Nanopore SERS Array, Small, 2010, 6, p. 1741-1744.

17. Pan, Z., Zavalin, A., Ueda, A., Guo, M., Groza, M., Burger, A., Mu, R. and Morgan, S. H., Surface-Enhanced Raman Spectroscopy Using Silver-Coated Porous Glass-Ceramic Substrates, Appl. Spectrosc., 2005, 59, p. 782-786.

18. Zhang, L., Chang, H., Hirata, A., Wu, H., Xue, Q.-K. and Chen, M., Nanoporous Gold Based Optical Sensor for Sub-ppt Detection of Mercury Ions, ACS Nano, 2013, 7, p. 4595-4600.

19. Stewart, M. P. and Buriak, J. M., Chemical and Biological Applications of Porous Silicon Technology, Advanced Materials, 2000, 12, p. 859-869.

20. Janshoff, A., Dancil, K.-P. S., Steinem, C., Greiner, D. P., Lin, V. S. Y., Gurtner, C., Motesharei, K., Sailor, M. J. and Ghadiri, M. R., Macroporous pType Silicon Fabry-Perot Layers. Fabrication, Characterization, and Applications in Biosensing, Journal of the American Chemical Society, 1998, 120, p. $12108-12116$.

21. Menna, P., Di Francia, G. and La Ferrara, V., Porous silicon in solar cells: $A$ review and a description of its application as an AR coating, Solar Energy Materials and Solar Cells, 1995, 37, p. 13-24. 
22. Huang, Z., Fang, H. and Zhu, J., Fabrication of Silicon Nanowire Arrays with Controlled Diameter, Length, and Density, Advanced Materials, 2007, 19, p. 744-748.

23. Li, X. and Bohn, P. W., Metal-assisted chemical etching in $\mathrm{HF} / \mathrm{H}_{2} \mathrm{O}_{2}$ produces porous silicon, Applied Physics Letters, 2000, 77, p. 2572-2574.

24. Le Ru, E. C., Blackie, E., Meyer, M. and Etchegoin, P. G., Surface Enhanced Raman Scattering Enhancement Factors: A Comprehensive Study, The Journal of Physical Chemistry C, 2007, 111, p. 13794-13803.

25. Haynes, C. L. and Van Duyne, R. P., Plasmon-Sampled Surface-Enhanced Raman Excitation Spectroscopyt, The Journal of Physical Chemistry B, 2003, 107, p. 7426-7433.

26. Emory, S. R., Haskins, W. E. and Nie, S., Direct Observation of SizeDependent Optical Enhancement in Single Metal Nanoparticles, Journal of the American Chemical Society, 1998, 120, p. 8009-8010.

27. Jeanmaire, D. L. and Van Duyne, R. P., Surface raman spectroelectrochemistry: Part I. Heterocyclic, aromatic, and aliphatic amines adsorbed on the anodized silver electrode, Journal of Electroanalytical Chemistry and Interfacial Electrochemistry, 1977, 84, p. 1-20.

28. Xu, H., Aizpurua, J., Käll, M. and Apell, P., Electromagnetic contributions to single-molecule sensitivity in surface-enhanced Raman scattering, Physical Review E, 2000, 62, p. 4318-4324.

29. Schatz, G., Young, M. and Duyne, R., Electromagnetic Mechanism of SERS, in Surface-Enhanced Raman Scattering, K. Kneipp, M. Moskovits, and H. Kneipp, Editors. 2006, Springer Berlin Heidelberg. p. 19-45.

30. Schatz, G. C. and Van Duyne, R. P., Electromagnetic Mechanism of SurfaceEnhanced Spectroscopy, in Handbook of Vibrational Spectroscopy2006, John Wiley \& Sons, Ltd.

31. Hui, F., Yin, W., Jiahao, Z. and Jing, Z., Silver catalysis in the fabrication of silicon nanowire arrays, Nanotechnology, 2006, 17, p. 3768.

32. Huang, Z., Zhang, X., Reiche, M., Liu, L., Lee, W., Shimizu, T., Senz, S. and Gösele, U., Extended Arrays of Vertically Aligned Sub-10 nm Diameter [100] 
Si Nanowires by Metal-Assisted Chemical Etching, Nano Letters, 2008, 8, p. 3046-3051.

33. Serrano, A., de la Fuente, O. R. and Garcia, M. A., Extended and localized surface plasmons in annealed Au films on glass substrates, Journal of Applied Physics, 2010, 108, p. 074303-7. 


\title{
Chapter 5- Influence of Trimethylsilane Plasma \\ Coating on SERS Substrates and the
}

Commercialization Potential of Plasma Coated SERS

\author{
Substrates
}

\subsection{Introduction}

\subsubsection{Plasma nanocoating}

Plasma nanocoating technology is known as a powerful tool in surface modification and surface engineering of materials for various applications. Non-equilibrium plasmas or low-temperature plasmas, which can be easily created by electrical discharges under reduced pressures (e.g., 10 mtorr to 10 torr), are composed of highly reactive atomic, molecular, ionic and free radical species. When depositing gases such as organosilicons, hydrocarbons, or fluorocarbons are used, these highly reactive plasma species can develop ultrathin layers of 
plasma coatings in nanometer scale and tailor the surface chemistry/characteristics in accordance with the targeted functionalities. Typical application examples of plasma nanocoatings include significant improvement in adhesion, wettability, printability, corrosion resistance, and surface modification of nanomaterials [1-9]. In general, plasma nanocoating technology possesses many unique advantageous features including that: 1) plasma nanocoating technology can create a variety of desirable surface functionalities to tailor surface characteristics of various materials for desired applications; 2) plasma nanocoatings are chemically inert and corrosion-resistant; 3) plasma deposition is a dry process with no hazardous effluent and therefore an environmental compatible technology.

The use of plasma nanocaotings in surface-enhanced Raman spectroscopy (SERS) substrate development is expected to present several benefits. First, compare to conventional overlayer materials, the ultrathin plasma nanocoatings are extremely stable to both aging and temperature due to their highly three dimensional crosslinking chemical structures. This helps to maintain the desired high stability of SERS activity with minimal decrease in sensitivity. Second, the plasma coating can be made optically transparent and do not interfere with SERS measurement. Third, the surface chemistry and surface 
properties of the plasma nanocoatings can be tailored and controlled by simply adjusting the plasma chemistry or plasma gas composition. The control of the surface chemistry will enable us to improve the surface affinity of specific analyte to SERS substrates and thus fabricate SERS active substrates with an analyte-specific affinity plasma nanocoating. In comparison with the previously used thiolate self-assembled monolayers (SAM) that are thermally unstable, plasma nanocoating when used in SERS substrate fabrication enjoys the capability in surface chemistry control for adjustable analyte affinity, greater physical and chemical stability, more complete surface coverage, and simplicity and reliability for large scale fabrication of the SERS substrates.

\subsubsection{Commercialization of SERS substrates}

Recently, SERS has attracted wide interests from a vast number of research areas owing to the rapid development of nanoscience and nanotechnology. Numerous micro and nano fabrication techniques have been explored in the past two decades in an attempt to obtain reliable and scalable SERS substrates, including electron beam lithography $[10-12]$, nanosphere lithography $[13,14]$, plating $[15,16]$, colloidal chemistry [17], chemical and physical roughening [18, 19], oblique angle deposition [20, 21], de-alloying [22, 23], etc. While 
great efforts have been made towards the real-world applications of SERS, there are still several technical and non-technical challenges that significantly impede the commercialization of SERS. First, most SERS substrates exhibit inconsistent activities, which is mainly due to the lack of surface structural/characteristic control and uniformity over the entire area of the substrate. It is also worthy of pointing out that sensitivity is important, but not as important as other parameters such as the repeatability, stability, and reliability in many real-world applications. Second, although many manufacturing methods have been proposed and used for fabrication of SERS substrates, reliable large scale (wafer scale) fabrication need to be achieved in order to reduce the manufacturing cost and deliver affordable economic SERS substrates. Third, it is also a common problem that a subtle change in substrate manufacturing process can lead to significant changes of the Raman signals; an ideal SERS substrate should also allow relatively large processing tolerance for reliable performance. At last, considering that the real-world samples/analytes might contain a mixture of different chemicals, improved selectivity of SERS substrates and/or a better mechanism for cross-referencing will be needed.

At present, there are a few vendors that have translated SERS technology into commercialized products. Among them, Klarite [24] who recently emerged with Renishaw Diagnostic is taking a leading 
role in SERS market. Although the lithography based Au array made by Klarite has demonstrated a satisfactory combination of sensitivity and reliability, the high cost of such product has limited itself from wide acceptance. Other SERS products are mostly launched by startups, such as Ag sol-gel based SERS Vials by Real-Time Analyzers [25], SERS Media by Agiltron [26], ink-jet printed SERS paper substrates by Diagnostic anSERS Inc. [27], etc. Despite the promising prospect for these cost-effective SERS solutions, the break out year of commercial SERS products is still on the way.

\subsubsection{Our aims}

In the first half of this chapter, we present a design that combines plasma nanocoating with regular SERS substrate fabrication, followed by a direct comparison between plasma coated and uncoated SERS substrates in terms of SERS performance and a brief summary of the influence of plasma coating on SERS substrates. As shown in Fig. 5.1, the plasma coated SERS substrate consists of three components: base wafer, SERS-active structure, and plasma nanocoating. Base material used in this design is Si wafer because of its readiness for processing and low cost for large scale production. The SERS-active nanostructures are achieved through Au capsulated $\mathrm{SiO}_{2}$ nanoparticles. Certain aggregation is introduced and well controlled by the addition of 


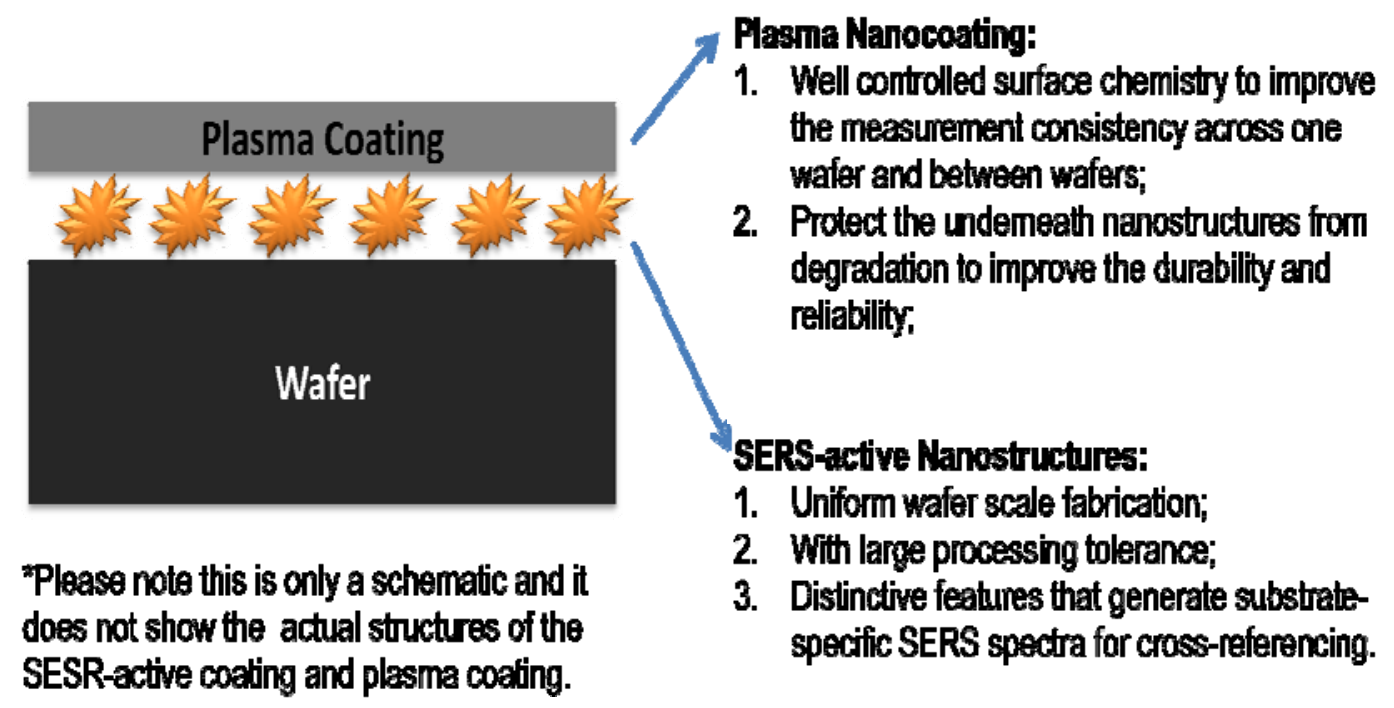

Figure 5.1. The design of combining plasma nanocoating with regular SERS substrates fabrication.

surfactants, which can not only improve the SERS sensitivity at small scale (a few nanometers) but maintain uniformity at large scale (a few hundred nanometers). On top of the SERS-active nanostructures, an innovational nanocoating is deposited with cold plasma, aiming to $\mathbf{1})$ control surface chemistry and improve measurement consistency and 2) protect the underneath nanostructures from degradation to improve the durability and reliability.

In the second half of this chapter, we evaluate the potential of commercializing plasma coated SERS substrate in several aspects, including a straight battle versus a commercial available product, repeatability, shelf life, applicability to different chemical species, and a real case study of detecting chemicals extracted from polluted fruits. 


\subsection{Experimental section}

\subsubsection{Fabrication of plasma coated SERS substrates}

$40 \mathrm{~nm} \mathrm{SiO} 2$ nanoparticles colloid (Nissan Chemical America Corporation) was diluted to $6 \mathrm{wt} \%$ in de-ionized (DI) water. $1 \mathrm{wt} \%$ polyvinylpyrrolidone (PVP, Sigma-Aldrich) was added into the above colloidal suspension as capping agent. Then the mixture was magnetically stirred for $2 \mathrm{~h}$ and ultrasonically treated to get satisfactory dispersion. The colloid was spun-coated (Laurell Technologies, WS-650sz-6NPP) onto Si wafer which had been rinsed by acetone, methanol and DI water in sequence and dried by nitrogen gas flow. The as-coated substrate was baked at $400{ }^{\circ} \mathrm{C}$ for $2 \mathrm{~h}$ in ambient oven to evaporate solvent residue, if any, and improve the adhesion between $\mathrm{SiO}_{2}$ nanoparticles and $\mathrm{Si}$ substrate. After baking, 40 $\mathrm{nm} \mathrm{Au}$ in mass thickness was deposited onto the $\mathrm{SiO}_{2}$ nanoparticles with an Emitech K650X sputtering tool. Please note, at this stage, SERS active substrates were already in place and they are referred to as SERS substrates in this work.

Plasma coating on as-prepared SERS substrates was carried out by room temperature plasma polymerization of trimethylsilane (TMS, United Chemical Tech). The process took place in a Pyrex bell-jar type plasma reactor. The internal electrodes were set up with two cathode 
electrodes and a grounded SERS substrate in the middle. After pumping down the reactor to its base pressure below 1 mTorr, reactive gas was introduced and controlled by $247 \mathrm{C}$ mass flow meter (MKS Instrument). The chamber pressure was monitored by a Baratron pressure meter and controlled by type 252 exhaust valve controller (MKS Instrument). Radio frequency (RF) power generator (13.56 MHz, RFX-600, Advanced Energy Industries) was used to initiate the glow discharge. Plasma treatment time was carefully controlled to ensure the coatings with desired thicknesses.

\subsubsection{Characterization of plasma coated SERS substrates}

The morphology of SERS substrates (pre and post plasma coating) was characterized by a Hitachi S-4700 field emission scanning electron microscope (FESEM). The plasma coating was examined with two aspects: (1) coating thickness and (2) surface free energy. Coating thickness was investigated by a null seeking-type AutoEL-II automatic ellipsometer with $632.8 \mathrm{He}-\mathrm{Ne}$ laser light source. The surface free energy was inferred from contact angle measurement that was conducted with sessile drop method. The contact angle of the water droplet on Si wafer surface was measured using a computercontrolled video capturing system (Video Contact Angle System, ASC products $2500 \mathrm{XE})$. 


\subsubsection{SERS measurement}

A Renishaw RM1000 Raman Spectrometer System equipped with a Leica DMLB microscope was used for this work. This system is equipped with a $785 \mathrm{~nm}$ near-infrared diode laser source. During the measurement, light from the high power (maximum: $300 \mathrm{~mW}$ ) diode laser was directed and focused onto the sample at a microscope stage through a $50 \times$ objective. Signals were detected by a $578 \times 385$ pixels CCD array detector. The size of each pixel was $22 \mu \mathrm{m} \times 22 \mu \mathrm{m}$. Spectra of samples were collected with $10 \mathrm{~s}$ exposure time, $0 \%$ focus, and $\sim 20 \mathrm{~mW}$ laser power in the extended mode. Detection range was set from 600 to $1700 \mathrm{~cm}^{-1}$. Unless otherwise noted, the Raman spectrometer is the default choice for this work.

In addition, a complementary portable Raman spectrometer (B\&W Tek i-Raman) was mainly used for the purposes of building chemicals database. The portable Raman system has a resolution of $4.4 \mathrm{~cm}^{-1}$ under the excitation of a $785 \mathrm{~nm}$ near-infrared diode laser source (maximum power at $330 \mathrm{~mW}$ ). Raman spectra were collected using a $20 \times$ objective with a $10 \mathrm{~s}$ exposure time and $\sim 50 \mathrm{~mW}$ laser output.

All chemicals involved were purchased from Sigma-Aldrich and used as received. All analytes were prepared in water and acetonitrile 
mixture (1:1 in volume). For each measurement, $0.2 \mu \mathrm{l}$ analyte was dropped onto SERS substrates and dried in air.

\subsubsection{Sample preparation for carbaryl detection from polluted fruits}

Pure 100 parts per million ( $\mathrm{ppm}$ ) carbaryl stock solutions were prepared using a mixed solvent system (acetonitrile : $\mathrm{H}_{2} \mathrm{O}=1: 1$, $\mathrm{v} / \mathrm{v})$. Solutions of $50,10,5,1,0.5$, and $0.1 \mathrm{ppm}$ carbaryl were prepared by serial dilutions from the $100 \mathrm{ppm}$ solution. The solvent without pesticides was used as the control.

Apples and tomatoes were weighed and their diameters measured. Assuming that an apple or a tomato is of spherical shape, surface area of a fruit was calculated. Using these data, given designated pesticide concentration on a fruit ( $\mathrm{ppm}$, or equivalently, $\mu \mathrm{g} / \mathrm{g})$, the mass of carbaryl $(\mu \mathrm{g})$ that should be spiked on one $\mathrm{cm}^{2}$ of fruit skin was obtained. Then certain amount of carbaryl solutions were dropped onto a piece of $\sim 4 \mathrm{~cm}^{2}$ fruit skin freshly peeled from the fruit with a pipette. Extra care was taken to ensure even distribution of the solution on the fruit skin. The skin sample was then blown dry, cut into smaller pieces, and put in a conical tube containing $4 \mathrm{~mL}$ of mixed solvent (acetonitrile : $\mathrm{H}_{2} \mathrm{O}=1: 1, \mathrm{v} / \mathrm{v}$ ). After vigorously vortexed for 1 min, the mixture was sonicated using an ultrasonic processor 
equipped with a $6.5 \mathrm{~mm}$ tapered microtip (Sonics \& Materials, Inc. Newtown, CT, USA) for 5 min with $30 \mathrm{~s}$ working and $10 \mathrm{~s}$ interval at an amplitude of $30 \%$. Finally, the supernatant was filtered with a $0.22 \mu \mathrm{m}$ syringe filter and the filtrate was used for SERS measurement.

\subsection{Results and discussion}

\subsubsection{Influence of plasma nanocoating on SERS substrates}

It should be declared that the thickness of plasma nanocoating was measured on bare Si wafers instead of actual SERS substrates because the working principle of ellipsometer isn't compatible with unsmooth surface. The thickness of plasma nanocoating was controlled

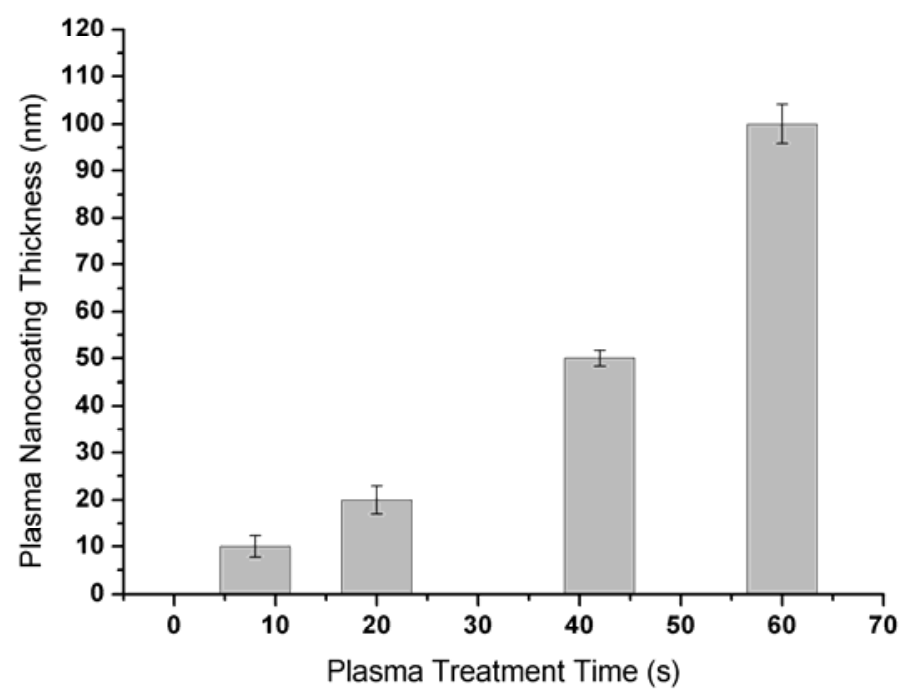

Figure 5.2. Thickness of plasma nanocoating on bare Si wafers with different plasma treatment time. 
by adjusting the plasma treatment time. As shown in Fig. 5.2, 8, 20, 42, and 60 s TMS plasma nanocoating ended up with four different thicknesses of $10,20,50$, and $100 \mathrm{~nm}$, respectively.

The contact angle measurement was conducted on actual SERS substrates. To calculate the change of surface energy after the plasma nanocoating, three types of solvents including HPLC grade water, diiodomethane, and ethylene glycol were used for the measurement of the contact angle. The surface energy was then calculated based on Lifshitz-van der Walls (LW)/acid-base (AB) approach.

$$
(1+\cos \theta) \cdot \gamma_{L}=2 \cdot\left(\sqrt{\gamma_{S}^{L W} \cdot \gamma_{L}^{L W}}+\sqrt{\gamma_{S}^{+} \gamma_{L}^{-}}+\sqrt{\gamma_{S}^{-} \gamma_{L}^{+}}\right)
$$

According to Eq. 1, unknown values $\gamma_{s}^{L W}, \gamma_{s}^{+}$, and $\gamma_{s}^{-}$were calculated from the known values $\gamma_{L}^{L W}, \gamma_{L}^{+}, \gamma_{L}^{-}$(Table 5.1) and measured value of $\cos \theta \cdot \gamma_{s}^{A B}$ was calculated by Eq. 2 and total surface energy was calculated by Eq. 3.

$$
\begin{aligned}
& \gamma_{s}^{A B}=2 \sqrt{\gamma_{s}^{+} \cdot \gamma_{s}^{-}} \\
& \gamma_{s}=\gamma_{s}^{L W}+\gamma_{s}^{A B}
\end{aligned}
$$

Table 5.1. Surface energy of liquid used to calculate surface energies of uncoated and TMS plasma coated SERS substrates. The units are $\mathrm{mJ} / \mathrm{m}^{2}$. Corresponding values are taken from the reference [28]. 


\begin{tabular}{lllll}
\hline & $\mathrm{YL}^{+}$ & $\mathrm{YL}^{-}$ & $\mathrm{YL}^{\mathrm{LW}}$ & $\mathrm{YL}$ \\
\hline Water & 25.5 & 25.5 & 21.8 & 72.8 \\
Diiodomethane & 0 & 0 & 50.8 & 50.8 \\
Ethylene glycol & 1.92 & 47.0 & 29.0 & 48.0 \\
\hline
\end{tabular}

As shown in Table 5.2, surface energy was controlled by the application of plasma nanocoating and tended to decrease as the nanocoating went to thick. For instance, surface energy of $100 \mathrm{~nm}$ TMS plasma coated SERS substrate equaled to $9.669 \mathrm{~mJ} / \mathrm{m}^{2}$.

Table 5.2. The contact angles $(\cos \theta)$ and their surface energies of uncoated and TMS plasma coated (thickness $=10,20,50,100 \mathrm{~nm}$ ) SERS substrates. Surface energy of each liquid is $\mathrm{YL}^{+}=25.5,0,1.92, \mathrm{YL}^{-}=25.5,0,47.0$, and $\mathrm{YL}^{\mathrm{LW}}=21.8,50.8,29.0$ for water, diiodomethane and ethylene glycol respectively.[28] $\mathrm{Ys}^{+}$and $\mathrm{Ys}^{-}$are the surface energies contributed from electron acceptors and donors. $\gamma^{\mathrm{LW}}$ and $\gamma^{\mathrm{AB}}$ are surface energies from lifshitz-van der Waals interaction and Lewis acid-base interaction.

\begin{tabular}{|c|c|c|c|c|c|c|c|c|}
\hline \multirow[b]{2}{*}{ SERS } & \multicolumn{3}{|c|}{ Contact angle $(\cos \theta)$} & \multicolumn{5}{|c|}{ Surface energy $\left(\mathrm{mJ} / \mathrm{m}^{2}\right)$} \\
\hline & Water & Diiodomethane & $\begin{array}{l}\text { Ethylene } \\
\text { Glycol }\end{array}$ & $\mathrm{Ys}^{+}$ & $\mathrm{Ys}^{-}$ & $\mathrm{Ys}^{\mathrm{LW}}$ & $\mathrm{Ys}^{\mathrm{AB}}$ & Ys \\
\hline Uncoated & 0.839 & 0.969 & 0.743 & 0.419 & 37.468 & 49.237 & 7.924 & $\begin{array}{l}57.16 \\
1\end{array}$ \\
\hline TMS 10nm & -0.515 & 0.087 & -0.044 & 0.0720 & 0.0334 & 15.006 & 0.0981 & $\begin{array}{l}15.10 \\
4\end{array}$ \\
\hline TMS 20nm & -0.508 & -0.0175 & -0.0523 & 0.176 & 0.532 & 12.259 & 0.612 & $\begin{array}{l}12.87 \\
1\end{array}$ \\
\hline TMS 50nm & -0.602 & 0 & -0.0349 & 0.306 & 0.0162 & 12.7 & 0.141 & $\begin{array}{l}12.84 \\
1\end{array}$ \\
\hline TMS 100nm & -0.571 & -0.139 & -0.216 & 0.0553 & 0.291 & 9.415 & 0.254 & 9.669 \\
\hline
\end{tabular}


A quick glance at the difference between uncoated and plasma coated SERS substrates is presented by the digital photograph of contact angle, shown in Fig. 5.3. The plasma nanocoating apparently altered the substrate surface to more hydrophobic. Microscopic investigation on uncoated and plasma coated SERS substrates was carried out with a FESEM. Figure 5.4(a) and (b) show the SEM images of SERS substrate before and after plasma nanocoating, respectively. Compared to the uncoated SERS substrate, plasma nanocoating clearly served as shell layer on top of the SERS-active nanostructures. In addition, the nanocoating seemed not uniform and might not have a full coverage on the underneath SERS-active

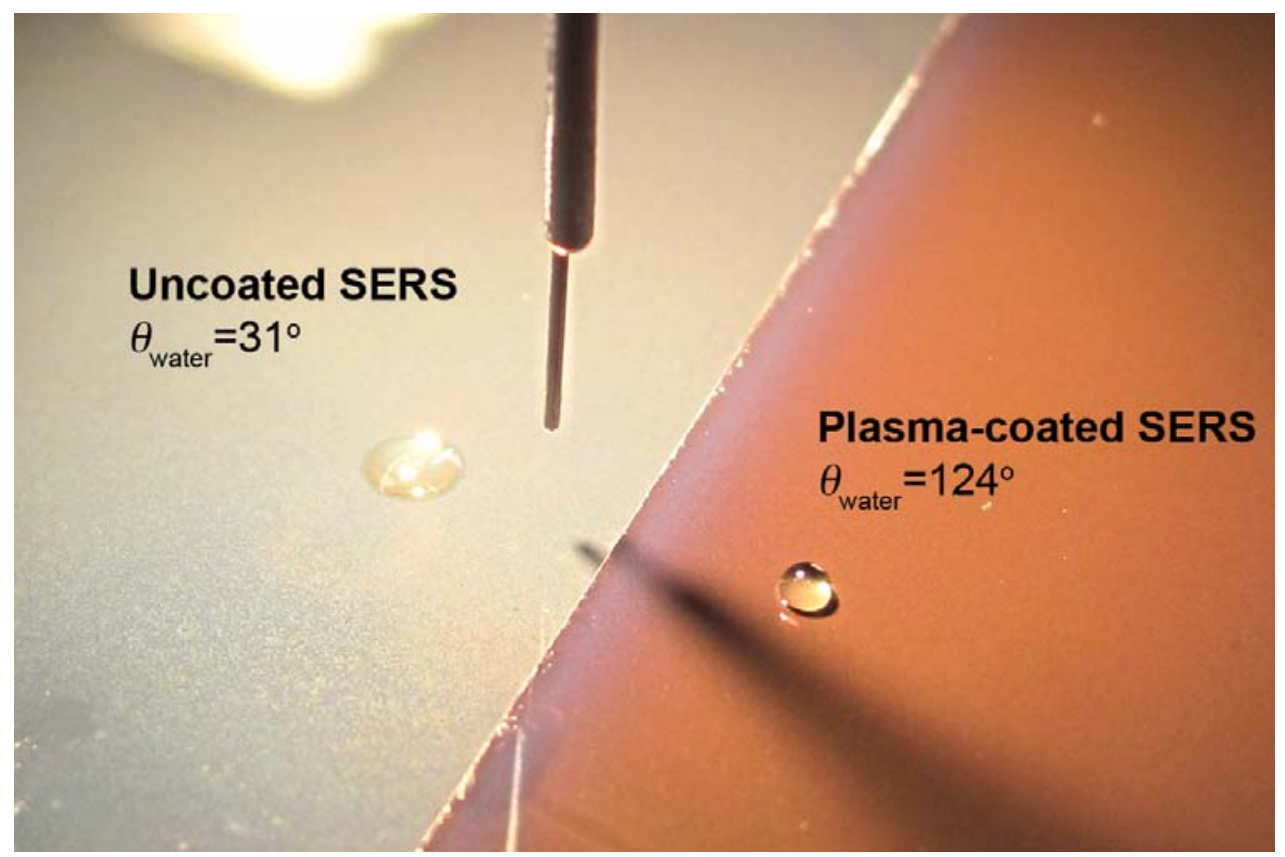

Figure 5.3. Digital photograph of water droplets on uncoated (left) and plasma coated (right, $50 \mathrm{~nm}$ in thickness) SERS substrates. 

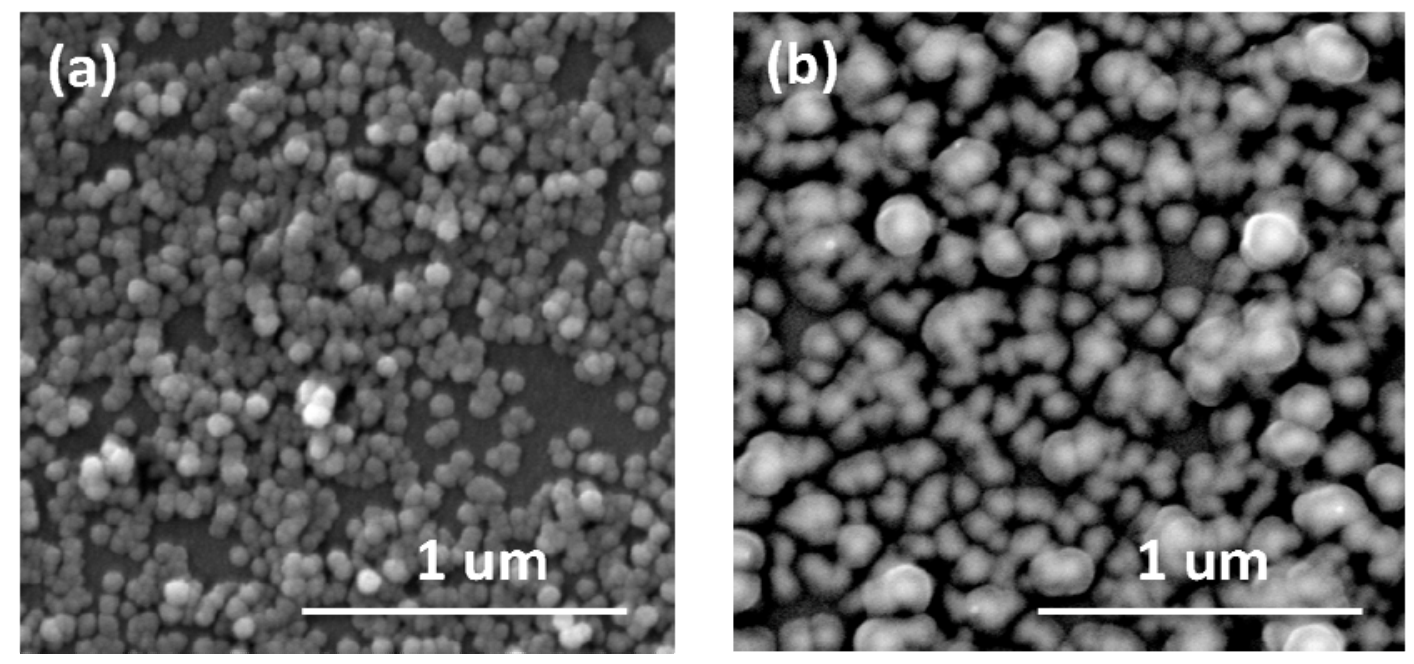

Figure 5.4. SEM images of SERS substrates before and after plasma nanocoating (50 $\mathrm{nm}$ in thickness).

nanostructures. It is very likely the nanocoating was primarily on the top of SERS-active nanostructures, leaving the sides of SERS-active nanostructures uncovered. This partial coverage feature of plasma nanocoating may play a critical role in SERS operation as it doesn't prevent analyte from percolating into the gaps, or so called SERS hot spots, among SERS-active nanostructures.

SERS detection of carbaryl, a chemical in carbamate family and mainly used as an insecticide, was performed using the uncoated and plasma coated SERS substrates described in Table 5.2. The representative Raman signal or peak intensity at $1379 \mathrm{~cm}^{-1}$ are shown in Fig. 5.5. It can be seen that the plasma nanocoating on SERS substrates improved the signal intensity over uncoated substrate. For instance, $100 \mathrm{~nm}$ plasma nanocoating raised the Raman signal by 


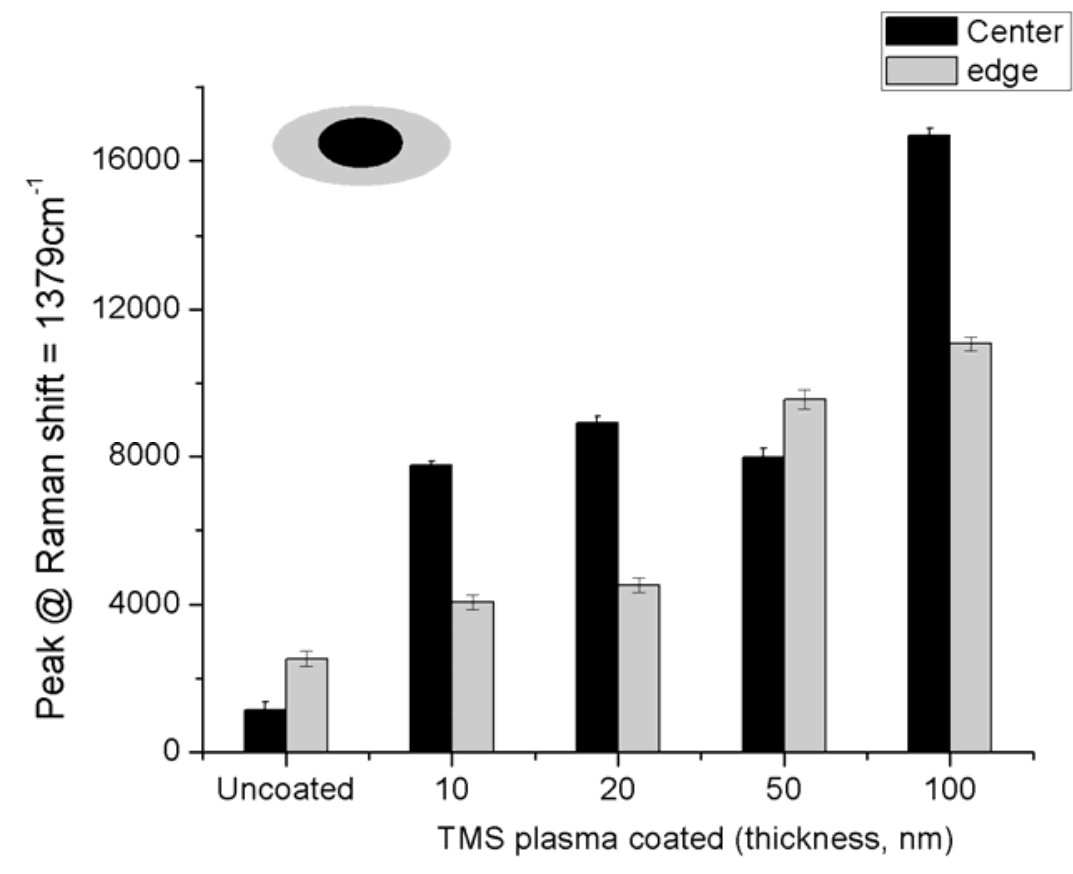

Figure 5.5. Relative peak intensity comparison at Raman shift of $1379 \mathrm{~cm}^{-1}$ using uncoated and plasma coated SERS substrates. The analyte was 100 ppm carbaryl in a solvent mixture of acetonitrile and deionized water (equal volume ratio). For each substrate, two measurements, one at the center and the other one at the edge of analyte residue area, were conducted.

about an order of magnitude compared with uncoated substrate. We have also observed very similar phenomena when using melamine as the analyte.

The increase of the peak intensity in Fig. $\mathbf{5 . 5}$ could be attributed to two possible mechanisms: 1) reduced droplet-substrate contact area and reduced residue area with higher concentration of analyte; and 2) improved adsorption of analyte on SERS-active substrates. The first mechanism, consistent with our observation, is also illustrated in Fig. 5.6. When the analyte (in solvent) has a very small contact angle 
(high surface energy) or easily wets the SERS surfaces, it generally will form a large and irregular residue. Variation of the contact angle may significantly influence the droplet to droplet residue concentration variations. On the contrary, the plasma nanocoating can deliver a surface with low surface energy that is hard to wet, leaving a small, regular and higher concentrated residue. In addition, plasma nanocoating ensures very uniform contact angle across a whole wafer and leads to more reproducible results. More importantly, the plasma coating makes the process more controllable. It is well accepted that an inorganic coating, if not permeable, could significantly degrade the enhancement factor, such as $1 \mathrm{~nm}$ of coating causing one order of magnitude signal decrease. However, we have not observed such degradation for the following possible reasons: 1 ) the coating is primarily on the top of Au nanostructure and may not fully cover the underneath SERS-active nanostructures, especially the side; 2) only some SERS-active nanostructure are coated; 3) the voids between plasma nanocoating make the coating permeable.

\subsubsection{Commercialization potential of plasma coated SERS substrates}

Since the inception of SERS, researchers have been exploring the opportunities for commercial applications of SERS. In the past 


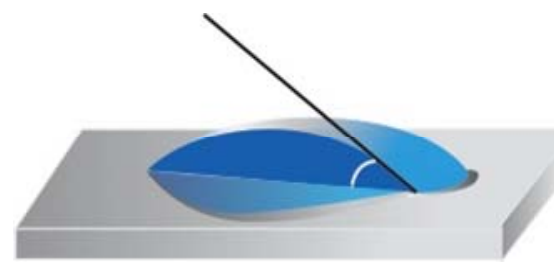

Uncoated SERS substrates

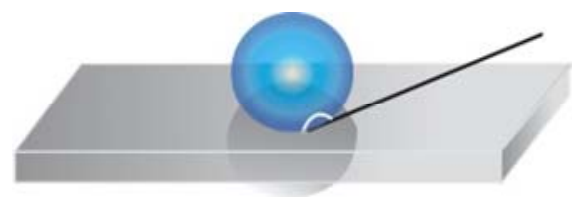

Plasma-coated samples

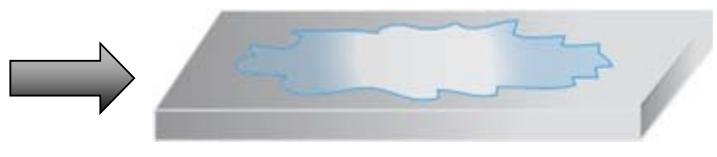

Lower and less reproducible residue concentration

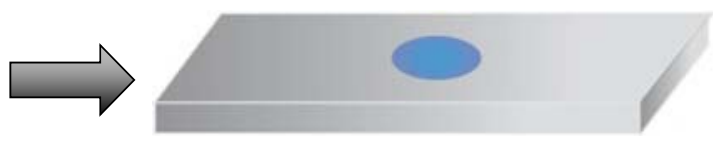

Higher and more reproducible residue concentration

Figure 5.6. Schematic illustration of the influence of plasma nanocoating on droplet shape and residue shape and size. Please note the laser beam size, $\sim 5 \mu \mathrm{m}$ in our case, is much smaller than the residue size.

decade, great efforts in doing so have been made and the dream has come into true as several commercial SERS products are already available on the market. However, the constraints imposed by a set of quality criteria (sensitivity, repeatability, scalability, cost and combinations of these) are limiting these products from making big and anticipated impact on real-world applications. Therefore, the SERS commercialization project is still in the early stage of development.

In this work, we evaluated the commercialization potential of plasma coated SERS substrates by directly comparing our substrates with a dominating commercial SERS product in terms of SERS sensitivity. Please note this SERS measurement was conducted on portable Raman spectrometer (B\&W Tek i-Raman). Figure $\mathbf{5 . 7}$ shows the SERS spectra of brilliant cresyl blue $(B C L)$ on plasma coated SERS 


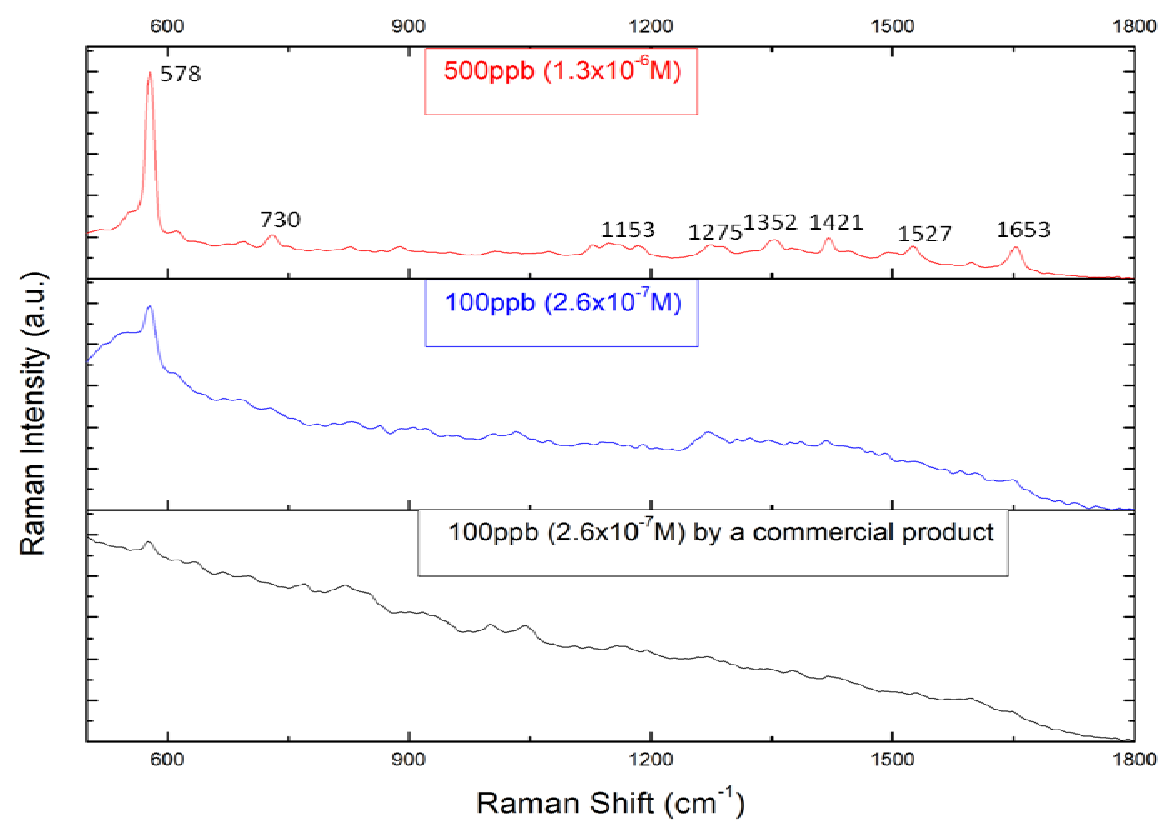

Figure 5.7. SERS spectra of $500 \mathrm{ppb}$ (red) and $100 \mathrm{ppb}$ (blue) $\mathrm{BCL}$ on plasma coated SERS substrate; black spectrum represents $100 \mathrm{ppb} B C L$ on a commercial SERS product.

substrate (red and blue curves) and the commercial SERS product (black curve). At high concentration of 500 parts per billion (ppb), plasma coated SERS substrate clearly exhibits all the characteristic peaks of $B C L$, indicating plasma coated SERS substrate is capable of completely presenting the targeted analytes. At low concentration of $100 \mathrm{ppb}$, both plasma coated SERS substrate and the commercial SERS product can deliver the main characteristic peak of $B C L$, though, other supplemental peaks are too weak to stand out. Based upon this representative comparison, we conclude that the plasma coated SERS substrates perform equally, at least, to the commercial SERS product 


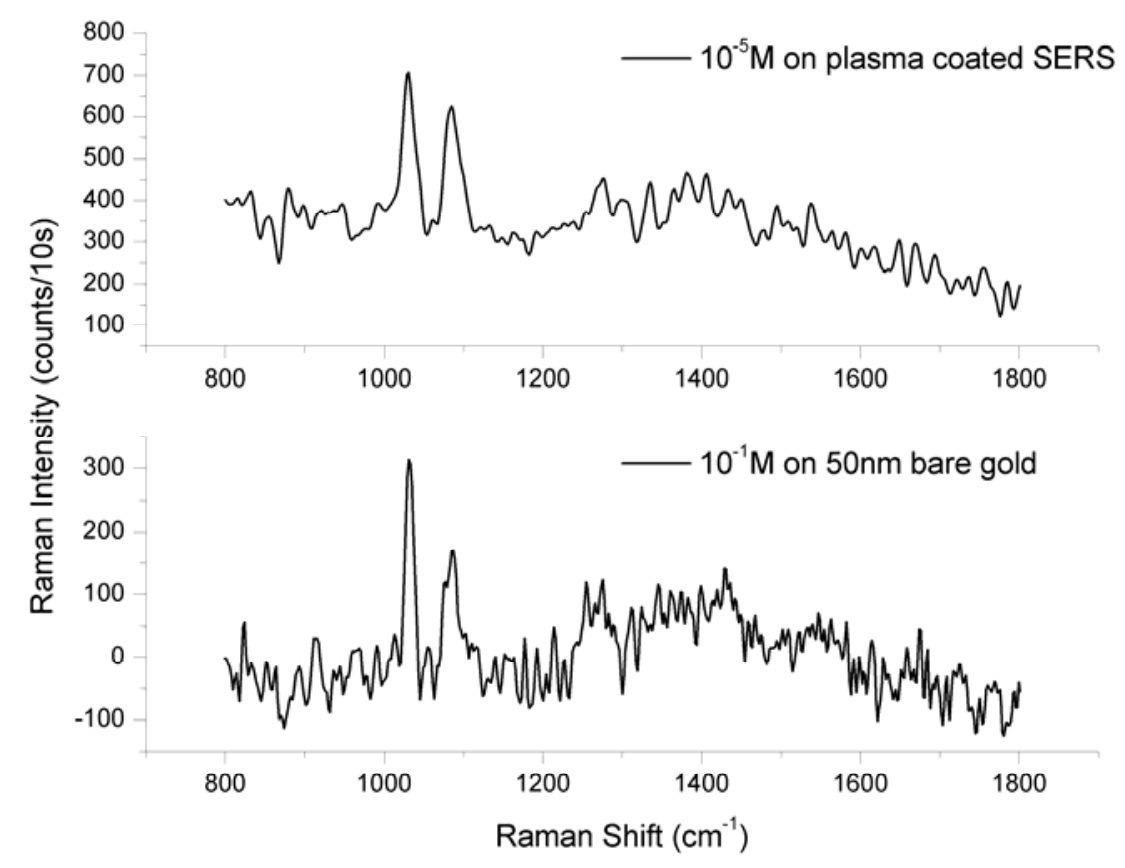

Figure 5.8. SERS spectrum of $10^{-5} \mathrm{M}$ BDT acquired on plasma coated SERS substrates (top). Raman spectrum of $10^{-1} \mathrm{M}$ BDT acquired on $50 \mathrm{~nm}$ Au film on un-etched Si (bottom).

in terms of sensing sensitivity. (Comparisons of other chemicals show similar results and are not listed here.)

In order to quantify the sensitivity of this plasma coated SERS substrates, measurement similar to previous chapters was conducted as well. Figure 5.8 shows the SERS spectrum of $10^{-5} \mathrm{M}$ BDT acquired from plasma coated SERS substrates and standard Raman spectrum of $10^{-1} \mathrm{M}$ BDT acquired from $50 \mathrm{~nm}$ bare Au films. According to analytical enhancement factor (AEF) definition, an AEF of $10^{4}$ over bare Au film is calculated for this plasma coated SERS substrates. 
To investigate the versatility of plasma coated SERS substrates, we tested a list of chemicals with plasma coated SERS substrates on both B\&W Tek and Renishaw platforms. The sensitivity of plasma coated and uncoated SERS substrates to these chemicals is listed in Table 5.3. Several points need be summarized about this table. First, for most chemicals, both plasma coated and uncoated SERS substrates are able to achieve ultrasensitive sensing at ppm level. Second, the competition between plasma coated and uncoated SERS substrates show mixed results, implying they are suited for different chemicals. But the difference between these two substrates is small overall. Last, the table indicates that Renishaw RM1000 unanimously outperforms B\&W Tek i-Raman by about 5-10 times in sensitivity, which is a straightforward outcome considering the former platform has a much better resolutions than the latter platform.

Table 5.3. Sensitivity of plasma coated and uncoated SERS substrates to various chemicals on two Raman spectrometers.

\begin{tabular}{|c|c|c|c|c|c|c|}
\hline \multicolumn{7}{|c|}{ Sensitivity } \\
\hline \multicolumn{3}{|c|}{ Chemicals } & \multicolumn{2}{|c|}{ Plasma Uncoated } & \multicolumn{2}{|c|}{ Plasma Coated } \\
\hline Name & CAS & Formula & $\begin{array}{c}\text { Portable } \\
\text { Raman }\end{array}$ & $\begin{array}{c}\text { Bench-top } \\
\text { Raman }\end{array}$ & $\begin{array}{l}\text { Portable } \\
\text { Raman }\end{array}$ & $\begin{array}{l}\text { Bench-top } \\
\text { Raman }\end{array}$ \\
\hline $\begin{array}{c}\text { 1,2-Bis(4- } \\
\text { pyridyl)ethane }\end{array}$ & $\begin{array}{c}4916- \\
57-8\end{array}$ & $\mathrm{C}_{12} \mathrm{H}_{12} \mathrm{~N}_{2}$ & $5 \mathrm{ppm}$ & $200 p p b$ & $10 \mathrm{ppm}$ & $1 \mathrm{ppm}$ \\
\hline $\begin{array}{l}4- \\
\text { Mercaptopyridine }\end{array}$ & $\begin{array}{c}4556- \\
23-4\end{array}$ & $\mathrm{C}_{5} \mathrm{H}_{5} \mathrm{NS}$ & 500ppb & 200ppb & $1 \mathrm{ppm}$ & 200ppb \\
\hline $\begin{array}{c}4- \\
\text { Methylbenzenethiol }\end{array}$ & $\begin{array}{c}106-45- \\
6\end{array}$ & $\mathrm{CH}_{3} \mathrm{C}_{6} \mathrm{H}_{4} \mathrm{SH}$ & $10 p p m$ & $2 \mathrm{ppm}$ & $10 \mathrm{ppm}$ & $2 \mathrm{ppm}$ \\
\hline Azinphos-methyl & $86-50-0$ & $\mathrm{C}_{10} \mathrm{H}_{12} \mathrm{~N}_{3} \mathrm{O}_{3} \mathrm{PS}_{2}$ & $2 \mathrm{ppm}$ & $5 p p m$ & $5 p p m$ & $5 p p m$ \\
\hline $\begin{array}{c}\text { Brilliant Cresyl } \\
\text { Blue }\end{array}$ & $\begin{array}{c}81029- \\
05-2\end{array}$ & $\mathrm{C}_{17} \mathrm{H}_{20} \mathrm{~N}_{3} \mathrm{OCl} \cdot 1 / 2 \mathrm{ZnCl}_{2}$ & 500ppb & $100 p p b$ & $100 p p b$ & $50 p p b$ \\
\hline Carbaryl & $63-25-2$ & $\mathrm{C}_{12} \mathrm{H}_{11} \mathrm{NO}_{2}$ & $10 p p m$ & $1 \mathrm{ppm}$ & $10 p p m$ & $1 \mathrm{ppm}$ \\
\hline Crystal Violet & $\begin{array}{c}548-62- \\
9 \\
\end{array}$ & $\mathrm{C}_{25} \mathrm{H}_{30} \mathrm{ClN}_{3}$ & 200ppb & $25 \mathrm{ppb}$ & $200 p p b$ & $25 p p b$ \\
\hline
\end{tabular}




\begin{tabular}{|c|c|c|c|c|c|c|}
\hline $\begin{array}{c}\text { Deiquat } \\
\text { Monohydrate }\end{array}$ & $\begin{array}{l}6385- \\
62-2\end{array}$ & $\mathrm{C}_{12} \mathrm{H}_{12} \mathrm{Br}_{2} \mathrm{~N}_{2} \cdot \mathrm{H}_{2} \mathrm{O}$ & 10ppm & $5 p p m$ & 50ppm & $5 p p m$ \\
\hline $\begin{array}{c}\text { Malachite Green } \\
\text { Chloride }\end{array}$ & $\begin{array}{l}569-64- \\
2\end{array}$ & $\mathrm{C}_{23} \mathrm{H}_{25} \mathrm{CIN}_{2}$ & $1 \mathrm{ppm}$ & $1 \mathrm{ppm}$ & 500ppb & $500 p p b$ \\
\hline Melamine & $\begin{array}{c}108-78- \\
1\end{array}$ & $\mathrm{C}_{3} \mathrm{H}_{6} \mathrm{~N}_{6}$ & $1 \mathrm{ppm}$ & $1 \mathrm{ppm}$ & $1 \mathrm{ppm}$ & 250ppb \\
\hline Phosmet & $\begin{array}{c}732-11- \\
6\end{array}$ & $\mathrm{C}_{11} \mathrm{H}_{12} \mathrm{NO}_{4} \mathrm{PS}_{2}$ & 10ppm & 500ppb & 25ppm & $5 p p m$ \\
\hline Rhodamine 6G & $\begin{array}{c}989-38- \\
8\end{array}$ & $\mathrm{C}_{28} \mathrm{H}_{31} \mathrm{~N}_{2} \mathrm{O}_{3} \mathrm{Cl}$ & $1 \mathrm{ppm}$ & $50 \mathrm{ppb}$ & $1 \mathrm{ppm}$ & $500 \mathrm{ppb}$ \\
\hline Sulfamethazine & $57-68-1$ & $\mathrm{C}_{12} \mathrm{H}_{14} \mathrm{~N}_{4} \mathrm{O}_{2} \mathrm{~S}$ & $1 \mathrm{ppm}$ & 200ppb & $5 p p m$ & 500ppb \\
\hline Thiram & $\begin{array}{c}137-26- \\
8\end{array}$ & $\left(\mathrm{CH}_{3}\right)_{2} \mathrm{NCSS}_{2} \mathrm{CSN}\left(\mathrm{CH}_{3}\right)_{2}$ & $5 \mathrm{ppm}$ & $2 \mathrm{ppm}$ & 10ppm & $2 \mathrm{ppm}$ \\
\hline
\end{tabular}

As mentioned earlier, repeatability is a key parameter to evaluate a SERS substrate, especially true when it comes to commercial application of SERS. In this work, we took a pair of paths to investigate the repeatability of plasma coated SERS substrates. In the first methodology, ten pieces of plasma coated SERS substrates $(0.5 \mathrm{~cm}$ by $0.5 \mathrm{~cm})$ were used to record SERS spectra of melamine at two concentrations (2.5 and $1 \mathrm{ppm})$. The intensity of characteristic peak at band of $683 \mathrm{~cm}^{-1}$ was used to characterize the measurement variation. As shown in the top part of Fig. 5.9, the peak intensities of ten plasma coated SERS substrates generally fall into the same level for a selected concentration of melamine with a relative standard deviation (RSD) of $21.1 \%$ for 1 ppm. In addition, all peak intensities of $2.5 \mathrm{ppm}$ melamine is as about 2 to 2.5 times as those of $1 \mathrm{ppm}$ melamine. In the second methodology, $1 \mathrm{ppm}$ melamine was tested on ten plasma coated SERS substrates $(0.5 \mathrm{~cm}$ by $0.5 \mathrm{~cm})$ by three operators. As indicated in the bottom part of Fig. 5.9, intensity 
variation indeed exists among three operators, but the result is overall consistent within each operator. The variation among three operators $(1277,1639$, and 1713$)$ is about $15.1 \%$, falling within the intrinsic RSD of plasma coated SERS substrates. In addition, there is another possible source for this variation among different operators. That is, it may be attributed to the different operational skills and habits. To be specific, as we already shown in Fig. 5.5, the SERS signal intensity is usually stronger at the center than the edge area of residue. We speculate the intensity variation among operators resulted from selecting different areas of residue. 

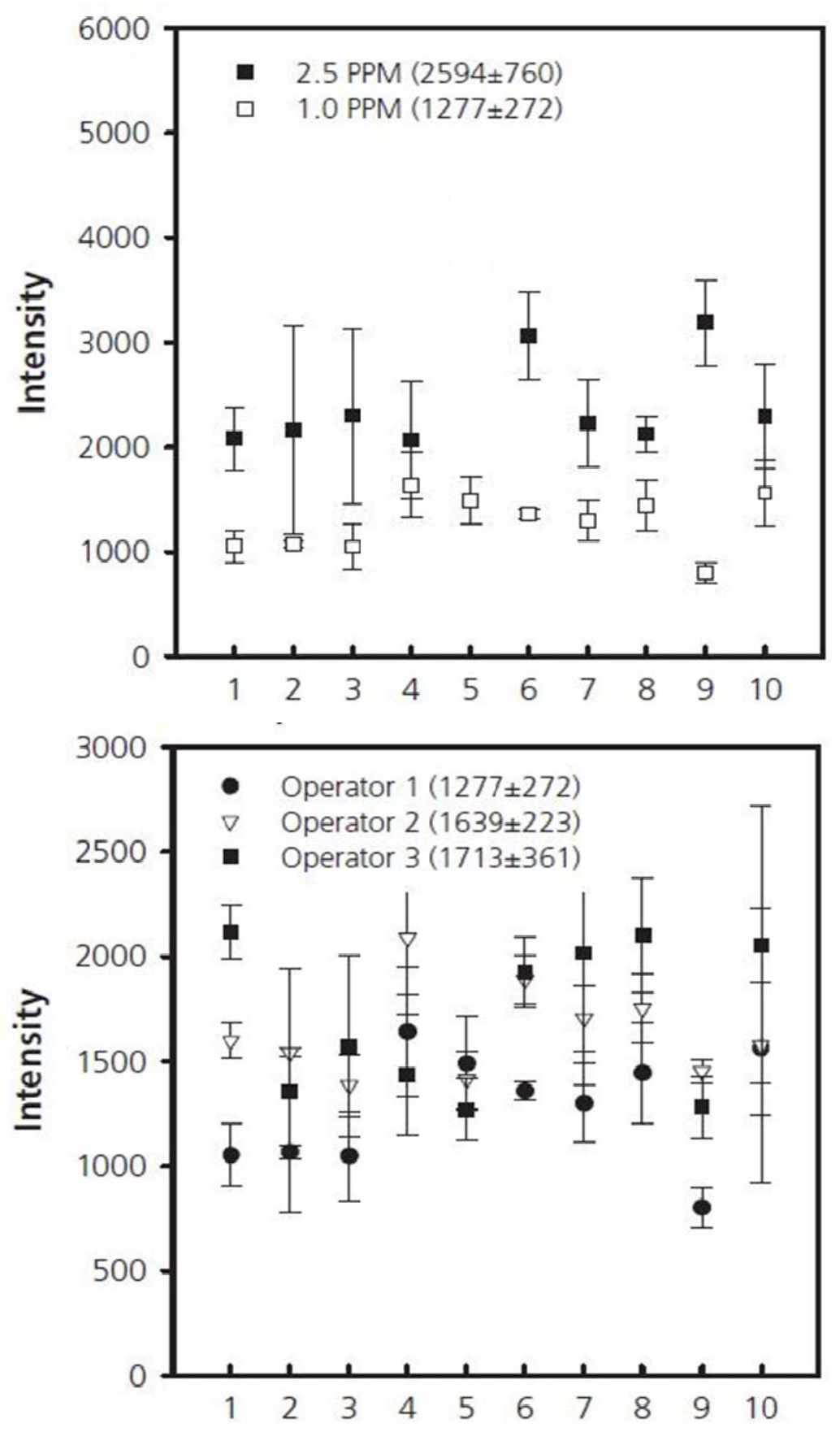

Figure 5.9. Top: Recorded peak intensities of $683 \mathrm{~cm}^{-1}$ ( 2.5 and $1 \mathrm{ppm}$ melamine) for ten plasma coated SERS substrates. Bottom: Recorded peak intensities of 683 $\mathrm{cm}^{-1}$ ( $1 \mathrm{ppm}$ melamine) on ten substrates that were operated by three operators.

Shelf life is also a very important factor for evaluating a SERS 
substrate. Our preliminary data shows that the nanostructures shown in Fig. 5.4(b) did not show any detectable degradation after exposed in ambient for up to 12 months. As confirmed in Fig. 5.10(a), the SERS spectra of $0.5 \mathrm{ppm}$ malachite green remain unchanged after different periods of aging, implying a long shelf life. Quantitatively, the peak intensity at $1173 \mathrm{~cm}^{-1}$ of these substrates was found to have a $27.7 \%$ RSD during aging. And the peak intensity goes up and down in the process instead of showing clear trend. Along with the fact that the substrate has an intrinsic RSD of $21.1 \%$, we speculate the aging effect is not obvious due possibly the protecting layer of plasma nanocoating. We also found that, very surprisingly, after a heat treatment at $700{ }^{\circ} \mathrm{C}$ in air for $2 \mathrm{~h}$, the plasma coated SERS substrates still shows similar SERS effects as if without heat treatment, while the uncoated substrates can no longer serve as a SERS substrate. We speculate that
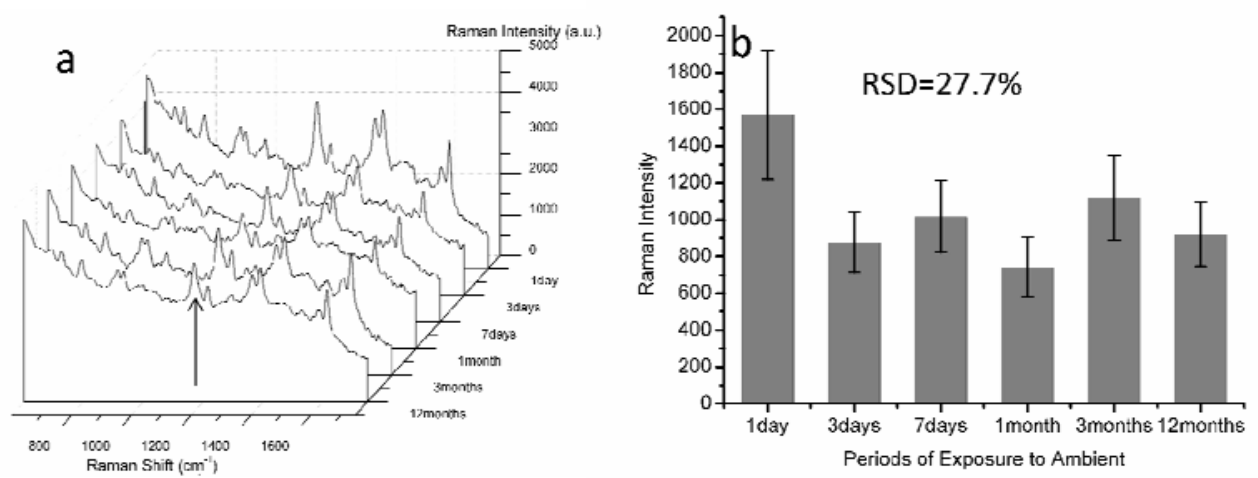

Figure 5.10. (a) SERS spectra of $0.5 \mathrm{ppm}$ malachite green on plasma coated SERS substrates after exposed in ambient for up to 12 months. (b) Raman intensity of a selected peak $\left(1173 \mathrm{~cm}^{-1}\right)$ after exposed in ambient for different periods. 
the uncoated $\mathrm{Au}$ nanostructures may experience a grain growth upon thermal attack, while the plasma nanocoating might serve as a cap layer that restrains surface diffusion and impedes the grain growth of Au nanostructures.

Finally, we wrap up the evaluation of plasma coated SERS

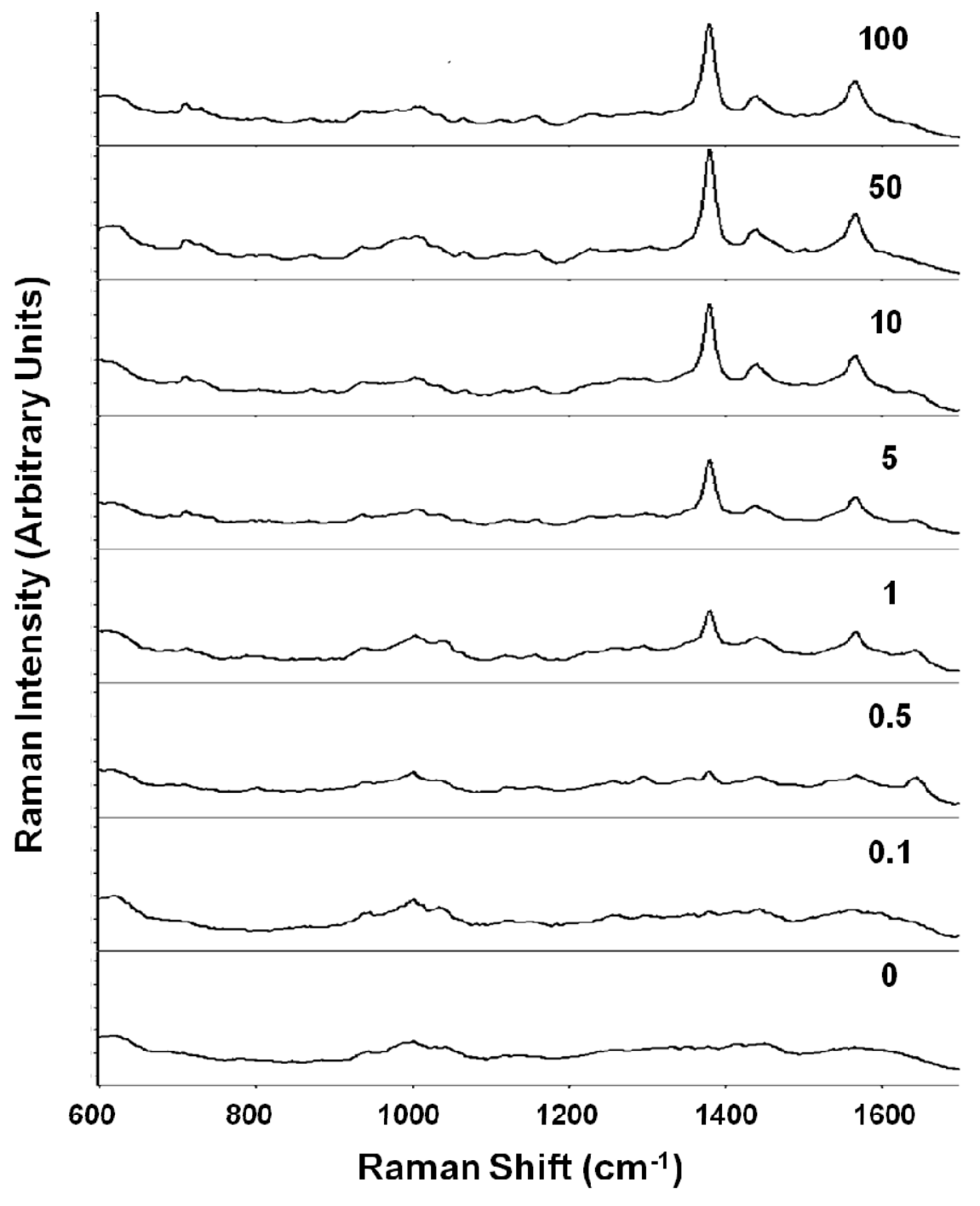

Figure 5.11. Average SERS spectra $(n=8)$ of carbaryl solutions with different concentrations (in ppm). Spectra were presented with smoothing at $4 \mathrm{~cm}^{-1}$ and baseline adjustment by subtracting a 2 nd order polynomial function. 
substrate's commercialization potential with a real case detection of carbaryl at trace amount level extracted from polluted fruits. As described in section 5.2.4, carbaryl of certain amount was purposely spiked into an apple or tomato to set up a polluted situation. Then we extracted the carbaryl from apple or tomato and tested it by plasma coated SERS substrate. According to the SERS spectra in Fig. 5.11, the plasma coated SERS substrate displays satisfactory detection capability as it is capable of detecting $0.1 \mathrm{ppm}$ carbaryl. We also used the same methodology to detect other types of pesticides and the details could be found elsewhere [29].

\subsection{Conclusion}

TMS plasma nanocoating has been applied onto the $\mathrm{Au} / \mathrm{SiO}_{2}$ SERS-active nanostructures. The thickness and surface energy of nanocoating were characterized by ellipsometry and contact angle method, respectively. The plasma nanocoating was found to 1) decrease surface energy of coated SERS substrates, particularly, with relative thick nanocoating, and 2) well control the size and shape of residue, leading to better SERS sensitivity and repeatability. SEM images reveal that plasma nanocoating sits primarily on top of the SERS-active nanostructures, leaving permeable regions at the sides of SERS-active nanostructures and ensuring the intactness of SERS "hot 
spots". In terms of SERS performance, preliminary chemical testing results indicate that plasma coated SERS substrates are more sensitive than the uncoated counterparts.

In addition, a series of study was carried out in order to evaluate the commercialization potential of plasma coated SERS substrates. That includes a direct comparison versus a commercial SERS product in terms of SERS sensitivity, the versatility for different chemicals, the repeatability among different substrates and different operators, the shelf life, and a real case study on chemicals extracted from polluted fruits. All measurement results suggest that the plasma coated SERS substrates meet the general quality criteria for SERS applications and are worth considerations for commercial plan.

\subsection{Reference}

1. Kim, Y. J., Ma, H. and Yu, Q., Plasma nanocoated carbon nanotubes for heat transfer nanofluids, Nanotechnology, 2010, 21, p. 295703.

2. Qingsong, Y., Kim, Y. J. and $\mathrm{Ma}, \mathrm{H}_{.}$, Plasma treatment of diamond nanoparticles for dispersion improvement in water, Applied Physics Letters, 2006, 88, p. Medium: X; Size: page(s) 231503-231503.3.

3. Shi, D., He, P., Lian, J., Wang, L. and Ooij, W. J. v., Plasma deposition and characterization of acrylic acid thin film on ZnO Nanoparticles, Journal of Materials Research, 2002, 17, p. 2555-2560.

4. Shi, D., Lian, J., He, P., Wang, L. M., Ooij, W. J. v., Schulz, M., Liu, Y. and Mast, D. B., Plasma deposition of Ultrathin polymer films on carbon nanotubes, Applied Physics Letters, 2002, 81, p. 5216-5218. 
5. Shi, D., Wang, S. X., Ooij, W. J. v., Wang, L. M., Zhao, J. and Yu, Z., Uniform deposition of ultrathin polymer films on the surfaces of $\mathrm{Al}_{2} \mathrm{O}_{3}$ nanoparticles by a plasma treatment, Applied Physics Letters, 2001, 78, p. 1243-1245.

6. Utracki, L. A., PLASMA DEPOSITION, TREATMENT, AND ETCHING OF POLYMERS edited by R. d'Agostino Academic Press, Inc., Boston 528 pages, hard cover, 1990, Materials and Manufacturing Processes, 1993, 8, p. 385 390.

7. Yasuda, H., ed. "Chapter 8. Deposition Kinetics", in Luminous Chemical Vapor Deposition and Interface Engineering. 2005, Marcel Dekker: New York.

8. Yasuda, H. K., Yu, Q. S., Reddy, C. M., Moffitt, C. E. and Wieliczka, D. M., Adhesion of spray primers to plasma polymer coatings, Journal of Applied Polymer Science, 2002, 85, p. 1443-1457.

9. $\mathrm{Yu}, \mathrm{Q} ., \mathrm{Kim}, \mathrm{Y} . \mathrm{J}$. and $\mathrm{Ma}, \mathrm{H} .$, Nanofluids with plasma treated diamond nanoparticles, Applied Physics Letters, 2008, 92, p. 103111-3.

10. Sackmann, M., Bom, S., Balster, T. and Materny, A., Nanostructured gold surfaces as reproducible substrates for surface-enhanced Raman spectroscopy, Journal of Raman Spectroscopy, 2007, 38, p. 277-282.

11. Billot, L., Lamy de la Chapelle, M., Grimault, A. S., Vial, A., Barchiesi, D., Bijeon, J. L., Adam, P. M. and Royer, P., Surface enhanced Raman scattering on gold nanowire arrays: Evidence of strong multipolar surface plasmon resonance enhancement, Chemical Physics Letters, 2006, 422, p. 303-307.

12. Yu, Q., Guan, P., Qin, D., Golden, G. and Wallace, P. M., Inverted SizeDependence of Surface-Enhanced Raman Scattering on Gold Nanohole and Nanodisk Arrays, Nano Letters, 2008, 8, p. 1923-1928.

13. Jensen, T. R., Malinsky, M. D., Haynes, C. L. and Van Duyne, R. P., Nanosphere Lithography: Tunable Localized Surface Plasmon Resonance Spectra of Silver Nanoparticles, Journal of Physical Chemistry B, 2000, 104, p. 10549-10556.

14. Ormonde, A. D., Hicks, E. C. M., Castillo, J. and Van Duyne, R. P., Nanosphere Lithography: Fabrication of Large-Area Ag Nanoparticle Arrays by Convective Self-Assembly and Their Characterization by Scanning UV-Visible Extinction Spectroscopy, Langmuir, 2004, 20, p. 6927-6931. 
15. Khan, M. A., Hogan, T. P. and Shanker, B., Gold-coated zinc oxide nanowirebased substrate for surface-enhanced Raman spectroscopy, Journal of Raman Spectroscopy, 2009, 40, p. 1539-1545.

16. Cheng, C., Yan, B., Wong, S. M., Li, X., Zhou, W., Yu, T., Shen, Z., Yu, H. and Fan, H. J., Fabrication and SERS Performance of Silver-NanoparticleDecorated Si/ZnO Nanotrees in Ordered Arrays, ACS Applied Materials \& Interfaces, 2010, 2, p. 1824-1828.

17. Jana, N. R. and Pal, T., Anisotropic Metal Nanoparticles for Use as SurfaceEnhanced Raman Substrates, Advanced Materials, 2007, 19, p. 1761-1765.

18. Williamson, T. L., Guo, X., Zukoski, A., Sood, A., Díaz, D. J. and Bohn, P. W., Porous GaN as a Template to Produce Surface-Enhanced Raman ScatteringActive Surfaces, Journal of Physical Chemistry B, 2005, 109, p. 20186-20191.

19. Christou, K., Knorr, I., Ihlemann, J. r., Wackerbarth, H. and Beushausen, V., Fabrication and Characterization of Homogeneous Surface-Enhanced Raman Scattering Substrates by Single Pulse UV-Laser Treatment of Gold and Silver Films, Langmuir, 2010, 26, p. 18564-18569.

20. Chaney, S. B., Shanmukh, S., Dluhy, R. A. and Zhao, Y. P., Aligned silver nanorod arrays produce high sensitivity surface-enhanced Raman spectroscopy substrates, Applied Physics Letters, 2005, 87, p. 031908-3.

21. Liu, Y., Fan, J., Zhao, Y. P., Shanmukh, S. and Dluhy, R. A., Angle dependent surface enhanced Raman scattering obtained from a Ag nanorod array substrate, Applied Physics Letters, 2006, 89, p. 173134-3.

22. Erlebacher, J., Aziz, M. J., Karma, A., Dimitrov, N. and Sieradzki, K., Evolution of nanoporosity in dealloying, Nature, 2001, 410, p. 450-453.

23. Biener, J., Nyce, G. W., Hodge, A. M., Biener, M. M., Hamza, A. V. and Maier, S. A., Nanoporous Plasmonic Metamaterials, Advanced Materials, 2008, 20, p. 1211-1217.

24. Klarite. Available from: http://www.renishawdiagnostics.com/en/klarite-serssubstrates--17076.

25. Real-Time Analyzers. Available from: http://www.rta.biz/Content/SERS Vials.asp. 
26. Agiltron.

Available

from:

http://www.ramansystems.com/raman\%20new\%20web/english/sers\%20enh ancing\%20media.htm.

27. Diagnostic anSERS Inc.; Available from: http://www.diagnosticansers.com/.

28. Matsushita, Y., Wada, S., Fukushima, K. and Yasuda, S., Surface characteristics of phenol-formaldehyde-lignin resin determined by contact angle measurement and inverse gas chromatography, Industrial Crops and Products, 2006, 23, p. 115-121.

29. Liu, B., Zhou, P., Liu, X., Sun, X., Li, H. and Lin, M., Detection of Pesticides in Fruits by Surface-Enhanced Raman Spectroscopy Coupled with Gold Nanostructures, Food and Bioprocess Technology, p. 1-9. 


\section{Chapter 6-Conclusion and Future Work}

\subsection{Conclusion}

In this dissertation, four plasmonic Au nanostructures were fabricated via different microfabrication approaches and characterized with microscopic techniques. First, faceted $\mathrm{ZnO} / \mathrm{Au}$ nanonecklace arrays (NN) were epitaxially grown on $r$-plane sapphire substrates by chemical vapor deposition, followed by sputtering. Second, Au nanoisland ( $\mathrm{NI}$ ) arrays with well controlled growth were prepared by repeated sputtering and post-deposition annealing. Third, nanoporous (NP) Si/Au composites were fabricated by metal-assisted wet etching and sputtering. Last, a novel plasma nanocoating (PN) technique was developed to overcoats SERS-active nanostructures and the benefits brought by such nanocoating was demonstrated. The properties and growth mechanisms of abovementioned plasmonic Au nanostructures were investigated with SEM, AFM, TEM, ellipsometry and contact angle analyzer. 
The potential of these plasmonic Au nanostructures for SERS applications was evaluated by a series of criteria, including repeatability, sensitivity (analytical enhancement factor, AEF), cost, scalability, shelf life and fabrication steps. Table 6.1 presents the summarized data of these plasmonic Au nanostructures in each category.

Table 6.1. Performance of four plasmonic Au nanostructures in reference to SERS evaluation criteria.

\begin{tabular}{|c|c|c|c|c|}
\hline Criterion & ZnO/ Au NN & Au NI & NP Si/ Au & PN SERS \\
\hline Repeatability & $\mathrm{n} / \mathrm{a}$ & $\begin{array}{c}7.0 \% \text { on } 5 \mathrm{~cm} \\
\times 5 \mathrm{~cm}\end{array}$ & $\begin{array}{c}4.4 \% \text { on } 1 \mathrm{~cm} \\
\times 1 \mathrm{~cm}\end{array}$ & $\begin{array}{l}21.3 \% \text { on } \\
150 \mathrm{~mm} \text { dia. }\end{array}$ \\
\hline AEF & $1 \mathrm{E}^{4}$ & $3 \mathrm{E}^{4}$ & $2 \mathrm{E}^{5}$ & $1 \mathrm{E}^{4}$ \\
\hline Substrate & $\begin{array}{l}\text { r-sapphire } \\
\$ 59 / \mathrm{in}^{2}\end{array}$ & $\begin{array}{l}\mathrm{Si}(100) \\
\$ 0.7 / \mathrm{in}^{2}\end{array}$ & $\begin{array}{l}\mathrm{Si}(100) \\
\$ 0.7 / \mathrm{in}^{2}\end{array}$ & $\begin{array}{l}\mathrm{Si}(100) \\
\$ 0.7 / \mathrm{in}^{2}\end{array}$ \\
\hline Energy & $900 \mathrm{C} 2 \mathrm{~h}$ & $200 \mathrm{C} 6 \mathrm{~h}$ & $200 C 2 h$ & $\begin{array}{l}\text { 400C } 2 \mathrm{~h} \\
\text { Plasma } \\
\text { Generator }\end{array}$ \\
\hline $\begin{array}{c}\text { Au } \\
\text { Coating }\end{array}$ & $40-60 n m$ & $40-60 n m$ & $>200 \mathrm{~nm}$ & 40-60nm \\
\hline Overall & High & Low & Low & Low \\
\hline
\end{tabular}




\begin{tabular}{|ccccc|}
\hline Scalability & $\sim 1000 \mu \mathrm{m}^{2}$ & $5 \mathrm{~cm} \times 5 \mathrm{~cm}$ & $1 \mathrm{~cm} \times 1 \mathrm{~cm}$ & $150 \mathrm{~mm}$ dia. \\
\hline Shelf Life & $\mathrm{n} / \mathrm{a}$ & $\mathrm{n} / \mathrm{a}$ & $\mathrm{n} / \mathrm{a}$ & $>12$ months \\
\hline $\begin{array}{c}\text { Fabrication } \\
\text { Steps }\end{array}$ & 4 & 7 & 4 & 5 \\
\hline
\end{tabular}

\section{Repeatability}

1) The repeatability test was not performed on $\mathrm{ZnO} / \mathrm{Au} \mathrm{NN}$ because the effective area on this sample was too small to conduct repeated signal acquisition. 2) Au NI shows promising repeatability on $5 \mathrm{~cm}$ by $5 \mathrm{~cm}$ sample. We did not test the repeatability on larger samples because this dimension already reaches the upper limit of our tool. 3) Repeatability of $\mathrm{NP} \mathrm{Si} / \mathrm{Au}$ is the highest among these nanostructures, but it is conducted on $1 \mathrm{~cm}$ by $1 \mathrm{~cm}$ sample. Future work is needed to conclude if the repeatability gets worse with enlarged sample. 4) Though the repeatability of PN SERS looks discouraging, it needs to be noted that this test is conducted on 150 $\mathrm{mm}$ wafer scale.

\section{Sensitivity}

All the AEF measurement is conducted with bare and flat Au film as reference. Among these nanostructures, NP $\mathrm{Si} / \mathrm{Au}$ exhibits the highest sensitivity, followed by $\mathrm{Au} \mathrm{NI}$, PN SERS, and ZnO/Au NN in 
sequence, and the difference among last three nanostructures is limited.

\section{Cost}

The key factor in cost analysis is from the perspective of wafer substrate choice. With $r$-plane sapphire wafer being greatly pricier than Si wafer, along with the higher processing temperature, fabrication of $\mathrm{ZnO} / \mathrm{Au} \mathrm{NN}$ apparently costs much more than the manufacturing of other three. As for comparison among Au NI, NP $\mathrm{Si} / \mathrm{Au}$ and PN SERS, the factors of energy and Au do not play significant roles because the consumptions of these two resources are in small amount.

\section{Scalability}

1) SEM characterization shows only a small portion of sapphire surface is covered with NN, so the scalability is a serious issue for $\mathrm{ZnO} / \mathrm{Au} \mathrm{NN}$. 2) As we mentioned above, our tool limits the dimension of $\mathrm{Au} \mathrm{NI}$ sample to $5 \mathrm{~cm}$ by $5 \mathrm{~cm}$. But based on the mapping in Fig.

3.11, there is a strong indication that this recipe may be further scaled up. 3) The scalability test of NP Si/Au has yet been conducted on samples larger than $1 \mathrm{~cm}$ by $1 \mathrm{~cm}$. This subject will be addressed in 
future work. 4) PN SERS has been successfully demonstrated at 150 $\mathrm{mm}$ wafer scale.

\section{Shelf life}

Shelf life study has been performed only PN SERS and the result shows PN SERS is still effective after being exposed in ambient for 12 months.

\section{Fabrication steps}

1) Fabrication of $\mathrm{ZnO} / \mathrm{Au} \mathrm{NN}$ starts with the synthesis of $\mathrm{Au}$ catalysts, followed by spin coating, thermal CVD and sputtering. 2) Preparation of $\mathrm{Au} \mathrm{NI}$ appears time consuming as it involves repeated deposition and anneal process (four sputterings and three anneals), but the procedures themselves are simple to operate and repeat. 3) Production of NP Si/Au includes sputtering, anneal, etch and sputtering. 4) Manufacturing of PN SERS involves colloid preparation, spin coating, baking, sputtering and plasma nanocoating.

In summary, the super high cost and the mediocre performance in other categories have placed $\mathrm{ZnO} / \mathrm{Au} \mathrm{NN}$ on the no go list for future study; the other three plasmonic nanostructures have exhibited enough merits, namely, the scalability of $\mathrm{Au} \mathrm{NI}$, the sensitivity of NP 
$\mathrm{Si} / \mathrm{Au}$ and the shelf life of PN SERS, to earn considerations for further refinement.

\subsection{Recommendations and Future Work}

The work included in this dissertation has made a big step forward in exploring the large scale production and real-world applications of SERS. In order to further improve the SERS performance of our plasmonic Au nanostructures, we recommend following work for future study:

1. Au nanoisland arrays: Study the shelf life of Au nanoisland arrays. Further scale up the production with large capacity equipments and study the repeatability of larger scaled Au nanoisland arrays.

2. Nanoporous Si/ Au composites: Study the influences of pre-defined Au nanoisland arrays on the morphology of nanoporous $\mathrm{Si}$ and the corresponding SERS performance. Utilize different parameterized Au nanoisland arrays (for instance, single, double, and triple processed Au nanoisland arrays reported in Chapter 3 to prepare nanoporous Si and examine the corresponding SERS performance. Explore the scalability and determine the shelf life. 
3. Plasma coated SERS-active nanostructures: Characterize the composition of plasma nanocoating by X-ray photoelectron spectroscopy (XPS). Find a more convincing way to investigate if the plasma nanocoating fully or partially covers the underneath SERS-active nanostructures.

4. All plasmonic Au nanostructures: As we mentioned earlier, the analyte droplet leaves a non-uniform residue after evaporation, causing varied spectral response from different regions within a residue. In order to better control the residue, it would be highly useful to study 1) the interaction between analyte droplet and substrate surface and 2 ) the dynamics of droplet evaporating process (may need collaboration with thermal/fluid experts).

5. All plasmonic Au nanostructures: Simulate the aging environment, such as high temperature, high humidity, and so on, to expedite the shelf life study. 


\section{VITA}

Xin Sun was born in 1984 in Liaoning Province of China. After graduating from Liaoning Xiuyan Senior High School in 2002, Mr. Sun was admitted by Xi'an Jiaotong University, a prestigious college in China, where he earned his bachelor degree in Materials Science and Engineering. From May 2007 to May 2008, Mr. Sun continued his graduate study in Materials Science and Engineering program in Shanghai Jiaotong University, too, a top ranked academic institution in China. Since June 2008, Mr. Sun has been studying for his Ph.D degree in Mechanical and Aerospace Engineering in University of Missouri-Columbia. 